SLAC-264

UC-34D

$(\mathrm{T} / \mathrm{E})$

\title{
- THE MEASUREMENT OF THE HADRONIC CROSS SECTION IN ELECTRON-POSITRON ANNIHILATION*
}

\author{
Scott Clearwater \\ Stanford Linear Accelerator Center \\ Stanford University \\ - $\quad$ Stanford, California $\mathbf{8 4 3 0 5}$
}

November 1883

Prepared for the Department of Energy

under contract number DE-AC03-76SF00515

Printed in the United States of America. Available from the National Technical Information Service, U.S. Department of Commerce, 5285 Port Royal Road, Springfield, Virginia 22161. Price: Printed Copy A06, Microfiche A01.

* Ph.D. dissertation. 


\section{Acknowledgements}

First and foremost I would like to thank my thesis advisor, David Ritson, for his tireless efforts in impressing upon me an understanding and appreciation for good scientific work. Gaining some of his intuition has been one of the most enlightening experiences I have ever had.

I would also like to thank Stephen Kaye for many discussions concerning physics analysis and Kwong Lau for many discussions particularly concerning two photon physics.

Special thanks go to Keith Rich for designing and maintaining our excellent off-line data flow system and to Dave Wiser who is largely repsonsible for our superb on-line data management.

I would like extend my appreciation to Harvey Lynch for his astute suggestions regarding this thesis.

I would also like to thank the members of SLAC Group F and MAC Operations for their contributions to the experiment. 

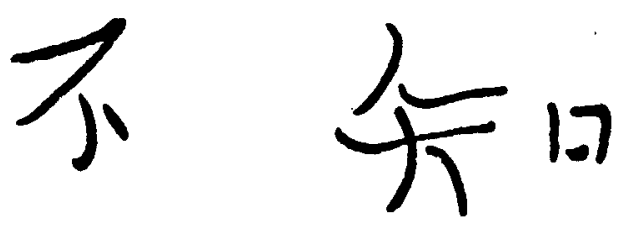

知
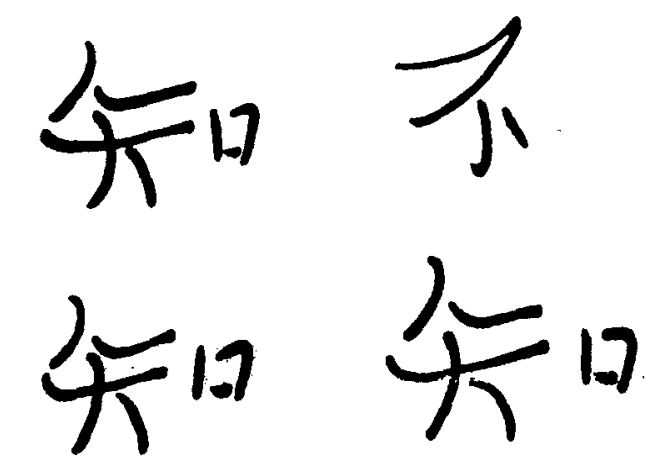

$=\frac{1}{16}$

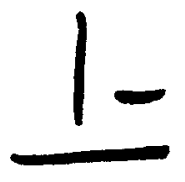

To know yet to think that one does not know is best;

Not to know yet to think that one knows will lead to trouble.

Lao Dzu

from the Dao De Jing 
Table of Contents

Chapter 1: Introduction .................

1.1 Particle Interactions $\ldots \ldots \ldots \ldots$

1.2 Experimental Remarks . . . . . . . . . . 12

Chapter 2: The MAC Detector $\ldots \ldots \ldots \ldots \ldots$

2.1 Drift Chambers . . . . . . . . . . . 20

2.2 The MAC Central Drift Chamber . . . . . . . 20

2.3 Calorimetry . . . . . . . . . . . 23

2.4 The MAC Electromagnetic Shower Chamber . . . . . . 26

2.5 The Scintillation Counter System . . . . . . . . . 28

2.6 The MAC Hadron Calorimeter . . . . . . . . 20

2.7 The Muon Tracking System . . . . . . . . . . 30

2.8 Running the Experiment $\ldots \ldots \ldots \ldots \ldots$

2.8 Comparison with Other Detectors . . . . . . . . 31

2.10 A Typical Multi-hadron Event in MAC . . . . . . 33

Chapter 3: Data Acquisition System . . . . . . . . . 35

3.1 Hardware Trigger System . . . . . . . . . . . . 35

3.2 The MAC Online Software Filter . . . . . . . . 42

3.3 Ofline Filter $\ldots \ldots \ldots \ldots \ldots \ldots \ldots$

Chapter 4: Data Analysis ................. 45

4.1 Introduction . . . . . . . . . . . 45

4.2 Evaluation of $\mathrm{R} \ldots \ldots \ldots \ldots$

4.3 Determination of $\mathrm{R} \ldots \ldots \ldots 8$ 
4.4 Comparison with Other Experiments . . . . . . 73

Chapter 5: Conclusions $\ldots \ldots \ldots \ldots \ldots \ldots \ldots$

Appendix A: Radiative Corrections $\ldots \ldots \ldots \ldots \ldots \ldots$

A.1 General Remarks . . . . . . . . . . . 78

A.2 Detailed analysis of $O\left(\alpha^{3}\right)$ and higher order contributions 80

A.3 Bhabha scattering . . . . . . . . 88

A.4 Multi-hadrons $\ldots \ldots \ldots \ldots . \ldots . \ldots 8$

A.5 Conclusion $\ldots \ldots \ldots \ldots \ldots \ldots$

Appendix B: Monte Carlo Methods . . . . . . . . . . 84

B.1 Multi-hadron Monte Carlos . . . . . . . . . 84

B.2 Detector Monte Carlo . . . . . . . . . 103

B.3 Using the Monte Carlos . . . . . . . . . . . 106

Appendix C: Luminosity Measurement . . . . . . . . . . 108

Bibliography .......................112 
List of Figures

1-1: QED diagrams to $O\left(\alpha^{3}\right) \ldots \ldots \ldots \ldots$

1-2: Lowest order QED diagrams of the $2 \gamma$ process $\ldots \ldots \ldots$

1-3: QCD diagrams to $O\left(\alpha_{s}^{2}\right) \ldots \ldots \ldots \ldots$

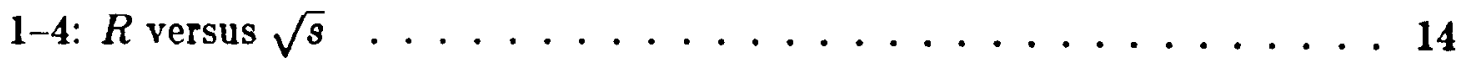

2-1: The MAC detector (end view) $\ldots \ldots \ldots \ldots$

2-2: The MAC detector (side view) $\ldots \ldots \ldots 18$

2-3: The MAC detector in the PEP storage ring . . . . . . . 19

2-4: Various angles in the central drift chamber $\ldots \ldots \ldots \ldots$

2-5: A typical multi-hadron event in MAC . . . . . . . . . 32

3-1: MAC data flow .................... 36

3-2: MAC data flow rates $\ldots \ldots \ldots \ldots \ldots \ldots$

4-1: Energy vs. Imbalance for various angular ranges $\ldots \ldots \ldots \ldots$

4-2: Charged multiplicity distributions $\ldots \ldots \ldots \ldots \ldots$

4-3: Energy spectrum for events with $>4$ charged tracks $\ldots \ldots \ldots \ldots$

4-4: Energy spectra for data and Monte Carlo events with $>8$ charged tracks60

4-5: Longitudinal vertex positions . . . . . . . . . 63

B-1: Bremsstrahlung spectrum $\ldots \ldots \ldots \ldots \ldots$

B-2: Quark fragmentation in the incoherent jet model $\ldots \ldots \ldots . . .99$ 
List of Tables

1-1: Comparison of QED, QCD, and Weak forces . . . . . . . . 12

4-1: Values of $\mathrm{R}$ for various angular cuts . . . . . . . . 54

4-2: Values of $R$ for various energy cuts $\ldots \ldots \ldots \ldots 1$

4-3: Estimates of the events passing the cuts $\ldots \ldots \ldots \ldots$

4-4: Change in acceptance due to variation in Monte Carlo parameters . . 67

4-5: Systematic errors in determing the acceptance $\ldots \ldots \ldots$

4-6: Final values and errors used in determining $R \ldots \ldots$

4-7: R-values at PEP and PETRA ........... 74

A-1: Vacuum polarization from vector meson resonances . . . . . . 81

A-2: Vacuum polarization from the hadron continuum $\ldots \ldots \ldots 2$

A-3: Radiative corrections to Bhabhas and multi-hadrons . . . . . . 84

A-4: Uncertainties in the leading $\log$ calculation $\ldots \ldots \ldots 8$

A-5: Uncertainty in $R$ due to low energy $R$-values $\ldots \ldots \ldots$

A-6: Final systematic errors due to radiative corrections $\ldots \ldots \ldots$. . . 92 


\section{Chapter 1}

\section{Introduction}

This thesis describes the most precise measurement to date of the ratio $R$, the hadronic cross section in lowest order electron-positron annihilation to the cross section for muon pair production in lowest order electron-positron annihilation usually defined as

$$
R=\frac{\sigma_{0}\left(e^{+} e^{-} \rightarrow \text { hadrons }\right)}{\sigma_{0}^{\mu \mu}}
$$

This experiment is of interest because $\mathbf{R}$ is a fundamental parameter that tests in a model independent way the basic assumptions of strong interaction theories. According to the assumptions of one of these theories the value of $\mathbf{R}$ is determined simply from the electric charges, spin, and color assignments of the produced quark-pairs.

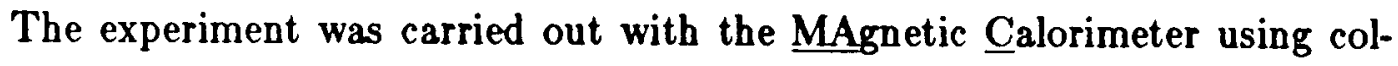
lisions of $14.5 \mathrm{GeV}$ electrons and positrons at the $2200 \mathrm{~m}$ circumference PEP storage ring at SLAC. The MAC detector is one of the best-suited collider detectors for measuring $R$ due to its nearly complete coverage of the full angular range. The PEP storage ring and the MAC detector have been in operation since 1979. Each year of PEP operation has produced a significant increases in collision 
rates. The data for this experiment were accumulated between February 1982 and April 1983 corresponding to a total event sample of about 40,000 hadronic events. About $5 \%$ of the data were taken with $14 \mathrm{GeV}$ beams and the rest of the data were taken with $14.5 \mathrm{GeV}$ beams. A description of particle interactions and experimental considerations follows.

\subsection{Particle Interactions}

The present theory of hadrons assumes that hadrons are composed of particles called quarks. For the following discussions it will be assumed that a multi-hadron final state is the result of a fragmentation cascade of an initial quark pair. The development of the quark theory will be discussed later in this chapter.

Electromagnetic Interactions

The extremely powerful results of Quantum Electrodynamics are used to model the electromagnetic interactions. The production of fermion-pairs in electron-positron collisions can proceed through two QED mechanisms. One is through electron-positron annihilation and the other is through the so-called two photon process.

In the annibilation process a fermion pair is created from the decay of a virtual photon. The cross section is computed using the Feynman rules for spinor electrodynamics. In the high energy limit $\left(E_{c m} \gg m_{f}\right)$ the angular dependence after summing over all polarizations becomes the well-known $1+\cos ^{2} \theta$ where 
$\theta$ is the angle of the outgoing particle relative to the beam axis. The actual differential cross section in the center-of-mass frame in the high energy limit is:

$$
\frac{d \sigma\left(e^{+} e^{-} \rightarrow f \bar{f}\right)}{d \Omega}=\frac{\alpha^{2} e_{f}^{2}}{4 s}\left(1+\cos ^{2} \theta\right)
$$

where the two factors of $\alpha$, the electromagnetic coupling constant $\left(\approx \frac{1}{137}\right)$, come from the two electromagnetic vertices, $e_{f}^{2}$ is the magnitude of the charge of the final state spin- $\frac{1}{2}$ particle divided by the magnitude of the electron charge, the factor $\frac{1}{8}$ ( 8 is the square of the center-of-mass energy) comes from the photon propagator, and $(h c)=1$. Higher order QED processes change the magnitude of the cross section considerably, $30-40 \%$ at PEP energies. See Appendix A for a detailed discussion of radiative corrections. The pertinent QED diagrams to $O\left(\alpha^{3}\right)$ are shown in Figure 1-1.

In the two photon $(2 \gamma)$ process an electron and positron from the incident beams emit virtual photons which in turn annihilate into a pair of particles. From the bremsstrahlung process there are two factors $\sim \alpha \ln \frac{E_{b e a m}}{m_{e}}$. From the photon-photon annihilation there are two more factors of $\alpha$. In the equivalent photon approximation ${ }^{1}$ the cross section for fermion pair production is given by:

$$
\sigma\left(e^{+} e^{-} \rightarrow e^{+} e^{-} f J\right) \approx \frac{112}{9 \pi} \alpha^{4} \frac{e_{f}^{4}}{m_{f}^{2}}\left(\ln \frac{E_{b e a m}}{m_{e}}\right)^{2} \ln \frac{E_{b e a m}}{m_{f}} .
$$

Although the $2 \gamma$ process is $O\left(\alpha^{4}\right)$, as $E_{\text {beam }}$ increases the log factors cause the $2 \gamma$ process to have a greater cross section than the annihilation process. The bremsstrablung photon angular dependence goes like:

$$
\frac{d \sigma_{b r e m}}{d \Omega} \sim \frac{1}{\sin ^{2} \theta_{b r e m}}
$$




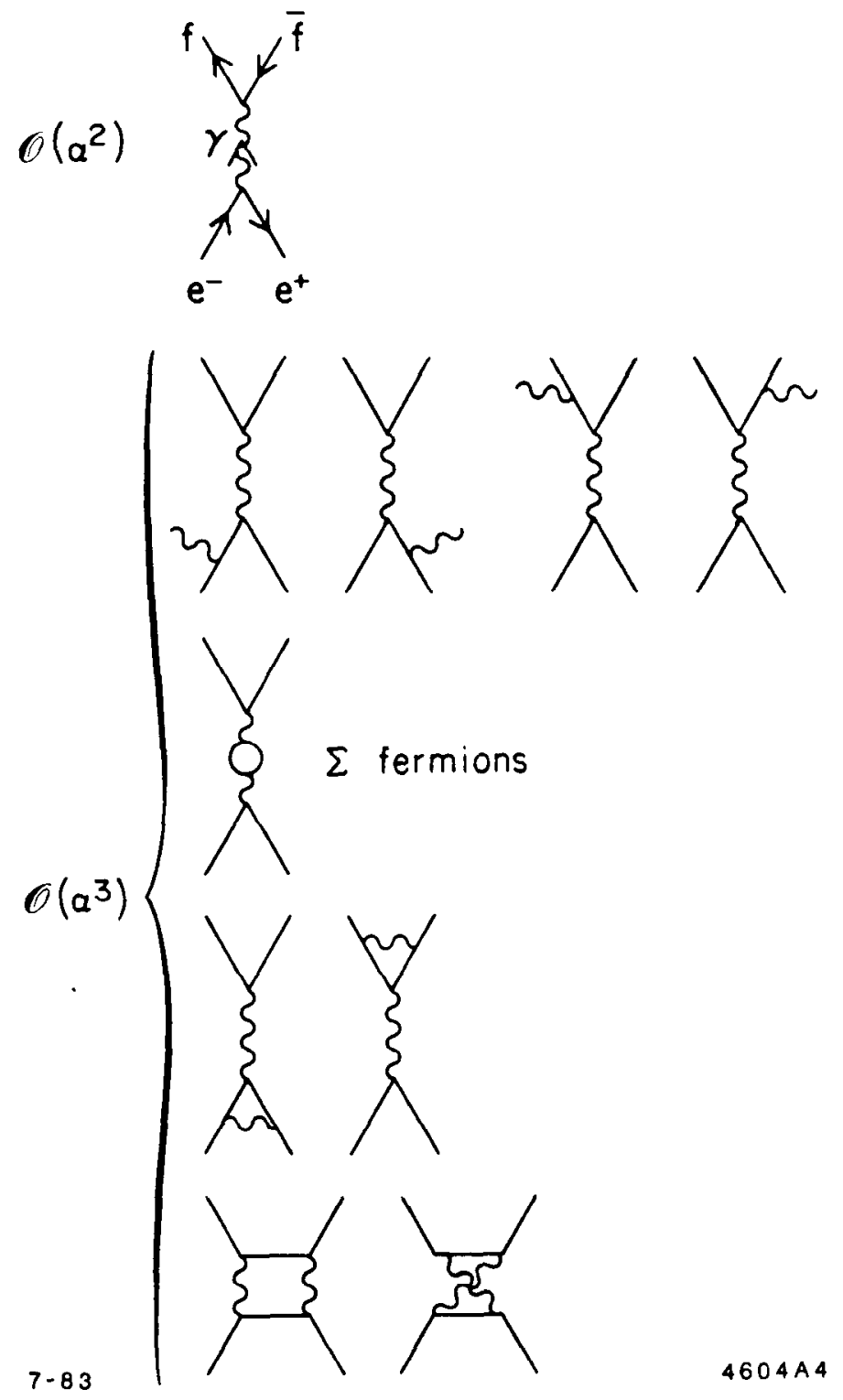

Figure 1-1: QED diagram of $c^{+} e^{-}$annihilation into a fermion pair to $O\left(\alpha^{3}\right)$. 
so that the center-of-mass motion in most $2 \gamma$ events is nearly along the beam line and in the case of multi-hadron final states the hadrons will also be mostly along the beam line. Also since the bremsstrahlung spectrum is:

$$
\frac{d \sigma}{d k} \sim \frac{1}{k}
$$

the center-of-mass energy of the photon-photon annihilation will be much smaller than in the annihilation of the beam particles, in this experiment about $3 \mathrm{GeV}$ versus $29 \mathrm{GeV}$. The six lowest order diagrams of the $2 \gamma$ process are shown in Figure 1-2.

\section{Weak Interactions}

The Standard Model for weak interactions allows fermion pair production to proceed through an intermediate vector boson, the $Z^{0}$. Because the weak coupling constant is small and the mass of the $Z^{0}$ is $\approx 00 \mathrm{GeV}$ the effect of the weak interaction on the total cross section is small at PEP energies. The change in $\mathrm{R}$ due to lowest order weak effects is written:

$$
\Delta R^{e-w}=3 \sum_{\substack{\text { guark } \\ \text { flavore }}} \frac{2 e_{q} g s\left(\frac{\theta}{M_{Z^{0}}^{2}}-1\right) v_{e} v_{f}-s^{2} g^{2}\left(v_{e}^{2}+a_{e}^{2}\right)\left(v_{f}^{2}+a_{f}^{2}\right)}{\left(\frac{s}{M_{Z^{0}}^{2}}-1\right)^{2}+\left(\frac{\Gamma_{Z^{0}}}{M_{Z^{0}}}\right)^{2}}
$$

where $e_{q}$ is the quark charge divided by the magnitude of the electron charge, $g \approx 4.47 \cdot 10^{-5} \mathrm{GeV}^{-1}$ is the weak coupling constant, $M_{Z^{0}} \approx 92 \mathrm{GeV}$, the width of the $Z^{0}$ is $\Gamma_{Z^{0}} \approx 2.5 \mathrm{GeV}$, and $v_{i}\left(a_{i}\right)$ is the vector (axial) coupling of particle $i$ with the $Z^{0}$. The change in $\mathrm{R}$ due to electro-weak interference is $\Delta R^{e-w} / R \approx$ $\frac{4}{10} \%$ at $\sqrt{8}=29 \mathrm{GeV}$.

$\underline{\text { Strong Interactions }}$ 

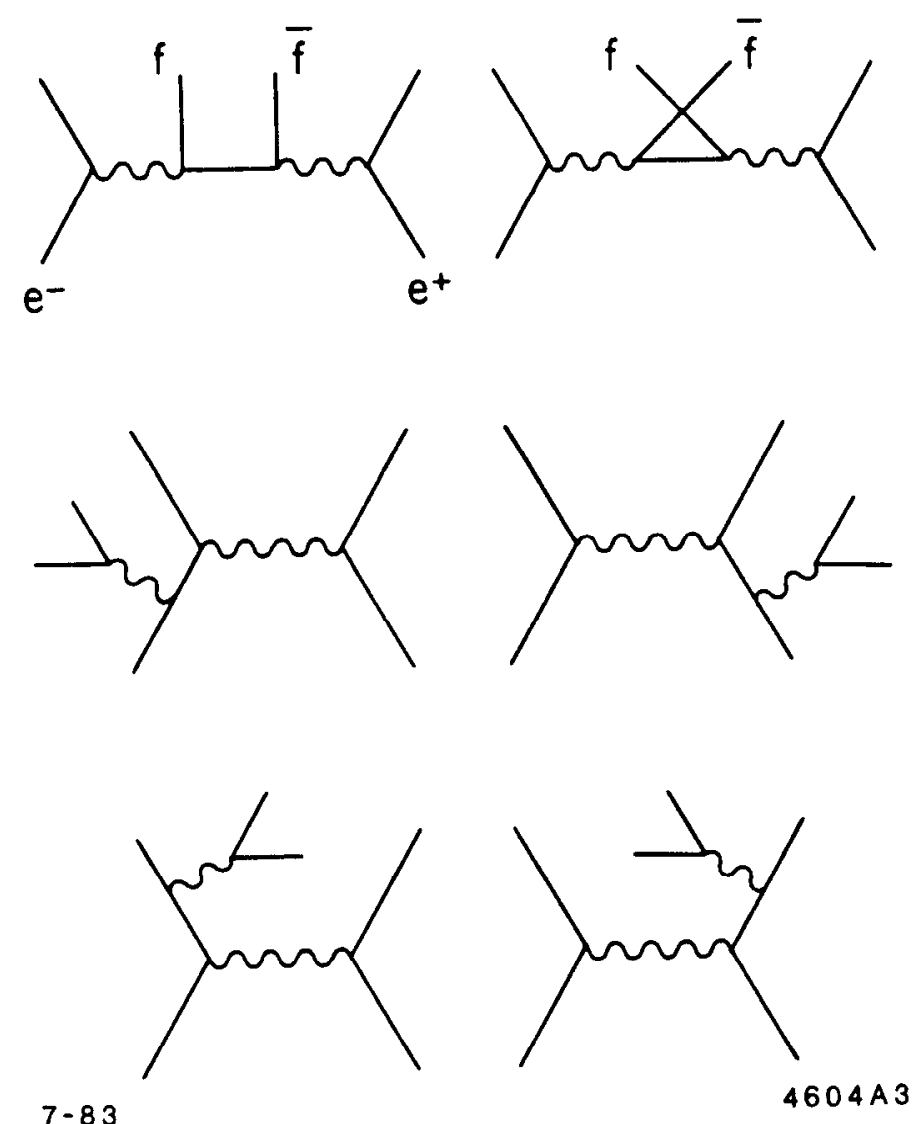

Figure 1-2: Lowest order QED diagrams of the $2 \gamma$ process. 
The quark model was developed as a means of explaining certain symmetries of the hadrons. Three different flavors (types) of quarks were originally postulated. Mesons contain a quark and an anti-quark, baryons contain 3 quarks. In order to avoid problems with Fermi statistics for certain hadrons a new property was postulated, color. Quarks can come in 3 different colors but all the observed hadrons are colorless (color singlets), that is the theory is an exact SU(3) color symmetry.

Later, experiments at SLAC involving deep inelastic scattering of electrons with protons led theorists ${ }^{2}$ to the idea that protons can be considered to be a composite of constituent particles called partons. The experimental evidence supporting this hypothesis was that the structure functions are a function only of the fractional momentum of the proton carried by the parton, a phenomenon referred to as Bjorken scaling. The parton model provides an intuitive picture of Bjorken scaling that neglects target mass effects, parton mass effects, interactions between partons, and the transverse parton momentum before the collision. This led theorists to explore asymptotically free theories (theories in which the coupling constant vanishes in some limit) as possible candidates for explaining the strong interaction. Asymptotic theories predict Bjorken scaling and the simple parton model.

The discovery that non-Abelian gauge theories are asymptotically free led to the present theory for the strong interaction, Quantum Chromo- Dynamics. The theory predicts asymptotic freedom and the possible confining of quarks and their quanta, gluons in the hadrons. QCD is also able to predict Bjorken scaling violations. One way to understand quark confinement is to think of a hadron as 
being immersed in a color dielectric. The color dielectric medium is the QCD vacuum and has dielectric constant $\approx 0$. This results in an anti-screening of color charge around the hadron. Using classical electrodynamics one can show that to move the quarks in a hadron infinitely far apart an infinite amount of energy is required, thus confining the quarks.

In terms of experiment asymptotic freedom can be investigated by measuring the strong coupling constant $\alpha_{d}$ which is a function of the momentum transfer, $q^{2}$, of the interaction. In particular as $q^{2}$ increases, the force and $\alpha_{s}\left(q^{2}\right)$ gets smaller, $i e$. we have asymptotic freedom.

The QCD prediction assumes that the quarks follow the canonical $1+\cos ^{2} \theta$ angular distribution of fermions. The axis of "momenta flow" or "thrust axis" of the final state hadrons closely follows the production angle of the primordial quarks. This means that using $Q C D, R$ can be expressed as the ratio of total cross sections or as the ratio of differential cross sections. As greater experimental precision can be achieved by using events confined in a central angular region our $\mathrm{R}$ measurement is based on the relation

$$
R=\frac{\int_{\theta_{\min }} \frac{d \sigma_{0}^{m h}}{d n} d \Omega}{\int_{\theta_{\min }} \frac{d \sigma_{0}^{\mu p}}{d \Omega} d \Omega}
$$

where $\theta_{\min }$ is the minimum polar angle over which the measurement is made.

The zeroth order $\mathrm{QCD}$ prediction for $\mathrm{R}$ is the same as that of the simple 
quark parton model and is written quite simply as:

$$
R=3 \sum_{\substack{\text { reserk } \\ \text { flavord }}} e_{q}^{2}
$$

where the factor of 3 is due to color and $e_{q}$ is the quark charge divided by the magnitude of the electron charge. At PEP energies, $\approx 30 \mathrm{GeV}$, the quark flavors are $u, d, s, c, b$ with relative charges $2 / 3,-1 / 3,-1 / 3,2 / 3$, and $-1 / 3$, respectively. The above values give a value of $3 \frac{2}{3}$ for $R$. Since the discovery of the $b$-quark there have been theoretical prejudices ${ }^{3}$ for a sixth quark, the top, or $t$-quark.

Assuming one is far from threshold effects, asymptotic freedom for the quarks permits a perturbative calculation of the interaction to be made. The fact that other measurements ${ }^{4-5}$ of $\mathrm{R}$ are close to the zeroth order calculation supports the perturbative assumption. Higher order QCD effects include gluon emission, gluon vertex correction and other diagrams shown in Figure 1-3. Using the modified minimal subtraction scheme $\mathbf{R}$ has been calculated for massless quarks to first order in $\alpha_{s}$, namely:

$$
R=3 \sum_{\substack{\text { quark } \\ \text { slavors }}} e_{q}^{2}\left[1+\frac{\alpha_{s}\left(q^{2}\right)}{\pi}\right]
$$

where $n_{f}$ is the number of quark flavors and $\alpha_{s}$ is given by

$$
\alpha_{s}\left(q^{2}\right) \approx \frac{12 \pi}{\left(33-2 n_{f}\right) \ln \left(q^{2} / \Lambda_{M S}^{2}\right)}
$$

where $\Lambda_{\overline{M S}}^{2} \approx 0.3 \mathrm{GeV}$ is an experimentally determined scale parameter. The QCD calculation of $R$ treats the quarks and gluons as if they are final state particles. The non-perturbative binding of quarks into hadrons is not taken into 


$$
\left.\theta\left(a_{s}\right)\{\}_{e^{-}}^{a a^{2 q} \bar{a}}\right\}
$$

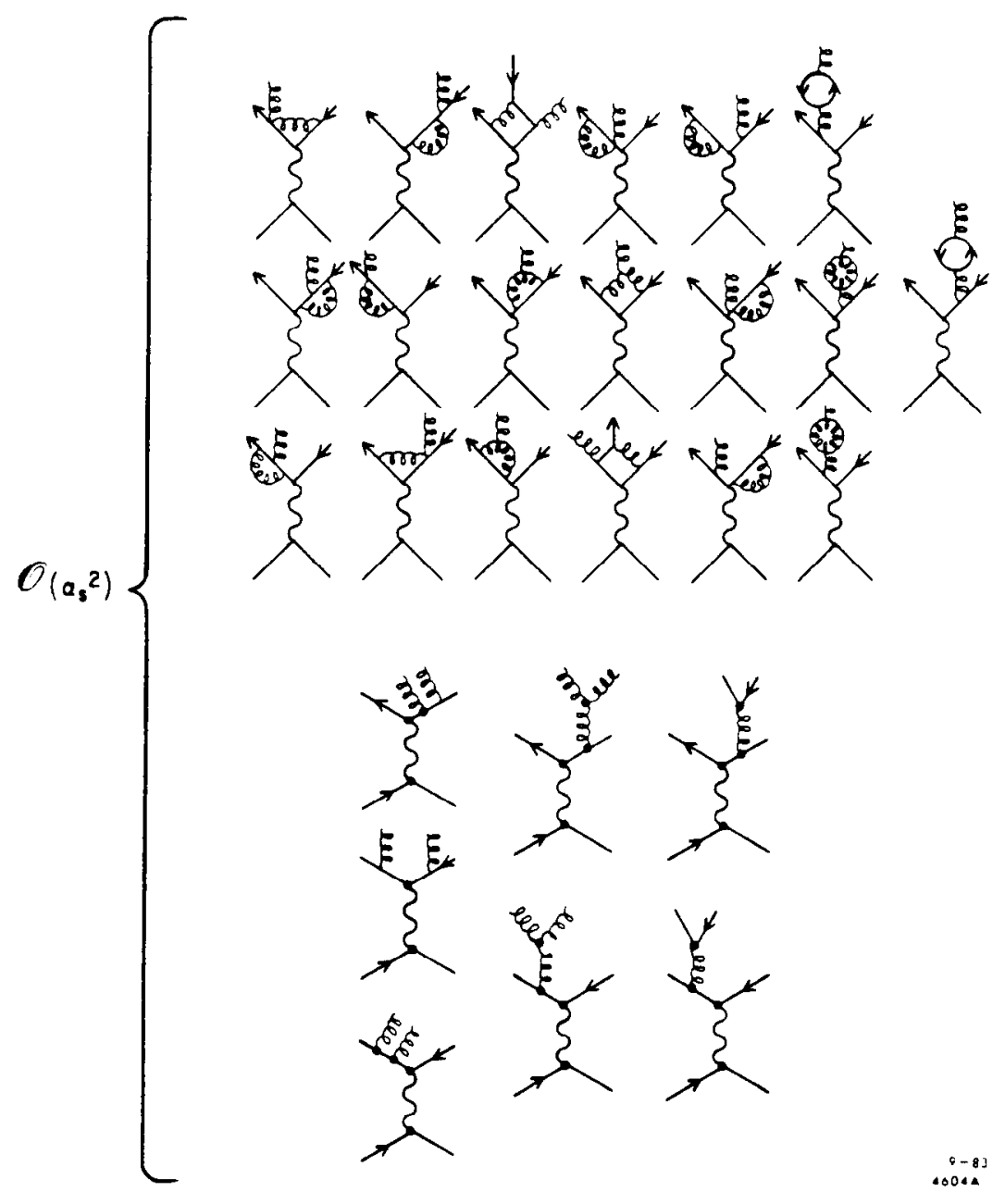

Figure 1-3: QCD diagrams in $e^{+} e^{-}$annihilation into quarks to $O\left(\alpha_{s}^{2}\right)$. 
account. As mentioned above the measured value of $R$ is close to the lowest order value (away from thresholds) indicating that hadronization is a process whose strength rapidly decreases with the interaction energy. Higher order calculations in $\alpha_{s}$ depend on the renormalization scheme.

As Eq. (1.1) shows the QCD calculation of $R$ is quite simple. Indeed there is only one scale parameter in the relation, $q^{2}$, which is fixed in $e^{+} e^{-}$experiments. Analysis of processes such as deep inelastic scattering depends on data compiled over a range of $q^{2}$ and parton momenta in order to see QCD effects. Also analyses in $e^{+} e^{-}$physics which involve jet studies such as three-jet analysis, and energyenergy correlations depend on the details of the hadronization process which is not calculable in perturbative QCD.

Once the primary quarks and possibly gluon(s) are produced, the quarks and gluons must "dress" themselves so that they become the particles actually seen in a detector. There are two basic models which are used to model the dressing process, these are the incoherent jet model and the string model both of which are explained in detail in Appendix B.

Table 1-1 highlights the differences between QED , QCD and the Standard Model of Weak interactions. 
Table 1-1

Comparison of QED, QCD, and the Standard Model of Weak Interactions QED QCD Standard Model Weak

$\begin{array}{lccc}\text { Propagator } & \text { photon } \gamma \text { gluon } g & Z^{0}, W^{ \pm} \\ \text {Coupling Constant } & \alpha & \alpha_{8} & g \\ \text { Gauge Group } & 0(1) & \mathrm{SU}(3) & \mathrm{SU}(2) \times \mathrm{U}(1)\end{array}$

\subsection{EXPERIMENTAL REMARKS}

Experimentally $R$ is evaluated via the expression:

$$
\frac{\int_{\theta_{\min }} \frac{d N^{m k}}{d \Omega} d \Omega}{\int_{\theta_{\min }} \frac{d \sigma_{0}^{\mu \nu}}{d \Omega} d \Omega \int L d t(1+\delta) \epsilon}=R
$$

where $\int_{\theta_{\min }} \frac{d N^{m h}}{d \Omega} d \Omega$ is the number of multi-hadron events from $e^{+} e^{-}$annihilation whose thrust axis is within the angular cut, $\delta$ is the radiative correction to lowest order annihilation including weak effects, $\int \mathcal{L} d t$ is the integrated luminosity (a quantity proportional to the $e^{+} e^{-}$collision rate) usually measured with large angle Bhabha scattering, $\epsilon$ is a correction factor to the acceptance of the experiment usually involving Monte Carlo techniques, and $\int_{\theta_{\min }} \frac{d \sigma_{0}^{\mu \mu}}{d \Omega} d \Omega$ is the lowest order QED differential cross section for $\mu$-pairs integrated over the same fiducial volume that the multi-hadrons are counted. The difficulty in the measurement comes in obtaining a very high purity sample of $1 \gamma$ (annihilation process) multi-hadrons, obtaining a low uncertainty measurement of integrated luminosity, and modeling the acceptance. This analysis has been carefully designed to reduce the above mentioned systematic uncertainties to a minimum. 
Measurements of $\mathrm{R}$ at $5-7 \mathrm{GeV}$ center-of-mass energy are systematically larger R-value than theory by about $5-15 \%{ }^{6}$ This discrepancy is much larger than the statistical uncertainty. Below $\sqrt{8}=5.5 \mathrm{GeV}$ the discrepancy is only 5 $8 \%$ which is within the systematic uncertainties. In the range $5.5 \mathrm{GeV}<\sqrt{s}$ $<7.8 \mathrm{GeV}$ the difference is $15-17 \%$ which is larger than the $10 \%$ systematic uncertainty. There is, then, a potential problem with $\mathbf{R}$ measurements between $5.5 \mathrm{GeV}$ and $7.8 \mathrm{GeV}$ which is indicative of a lack of knowledge of both theory and experiment.

The measurements of $R$ by the PETRA experiments ${ }^{7}$ in the energy range $\approx$ $15 \mathrm{GeV}<\sqrt{s}<37 \mathrm{GeV}$ are in good agreement with the QCD prediction within the $5-10 \%$ systematic errors of each experiment. In particular the $\mathbf{R}$ value is consistent with a constant in this energy range and is slightly above the simple quark model value. Figure 1-4 shows R-values in the energy range $5<\sqrt{s}<$ 36.7GeV. 


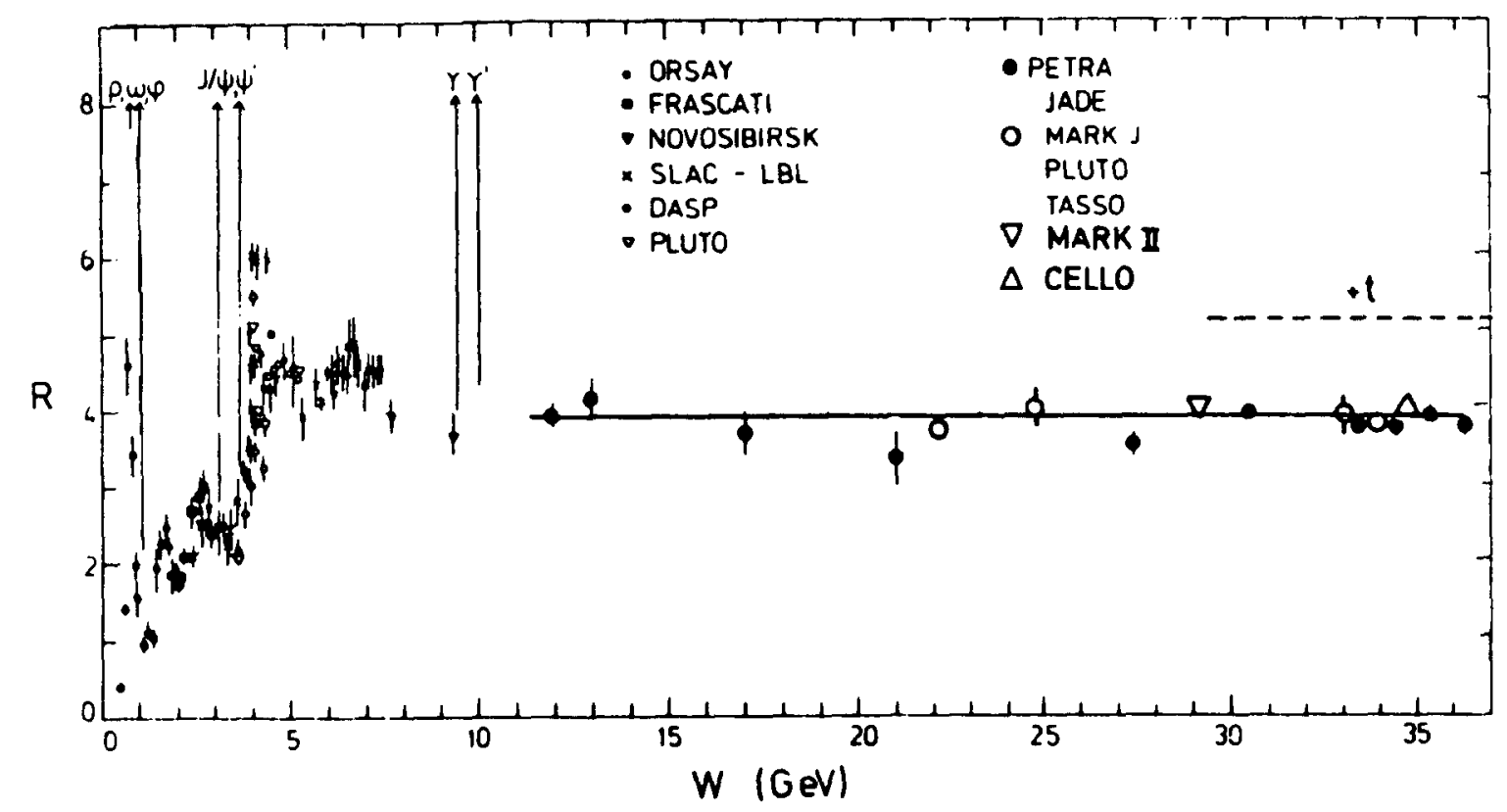

Figure 1-4: $R$ measurements in the energy range $5<\sqrt{8}<36.7 \mathrm{GeV}$. 


\section{REFERENCES}

1. Brodsky, S. J., T. Kinoshita, and H. Terazawa, Phys. Rev. D, 4, 1532 (1971).

2. Bjorken, J. D. and Pachos, G. A., Phys. Rev., 185, 185 (1869).

3. Kobayashi, M. and K. Maskawa, Progr. Theor. Phys.,49, 652 (1973).

4. Barber, D. P., et al., Phys. Rev. Lett., 42, 1113 (1979).

5. Brandelik, R., et al., Phys. Lett., 88B, 171 (1879).

6. Barnett, R. M., M. Dine, and L. McLerran, Phys. Rev. D, 22, 584 (1980).

7. Mess, K. H. and B. H. Wiik, in Recent Results in Electron-Positron and Lepton-Hadron Interactions, DESY 82-011 (1882). 


\section{Chapter 2}

\section{The MAC Detector}

The MAC detector ${ }^{1}$ is a general purpose detector featuring nearly full solid angle electro-magnetic/hadronic calorimetry. An inner drift chamber provides charged particle tracking and an outer drift system is used for muon analysis. Figure 2-1 and Figure 2-2 show the MAC detector.

In the MAC coordinate system the polar angle relative to the beam axis is measured in $\theta$, the azimuthal angle measured in a plane perpendicular the beam axis is measured in $\phi$, and longitudinal or axial measurements along the beam axis are measured in the coordinate $z$.

The MAC detector is situated in one of the six PEP interaction regions. Figure 2-3 shows the orientation of MAC in the PEP storage ring. The colliding beams of PEP contain particles not in the nominal beam orbit which results in radiation spraying into the detector creating so-called "blotch events". Some of the radiation is secondary radiation resulting from misfocused beam particles which have showered in material upstream from the detector. Another source of radiation is from beam particles which are over-focused by the quadrupole magnets near the interaction region. Additionally, synchrotron radiation from the bending arcs shines on the detector producing "hot spots" in the detector. 


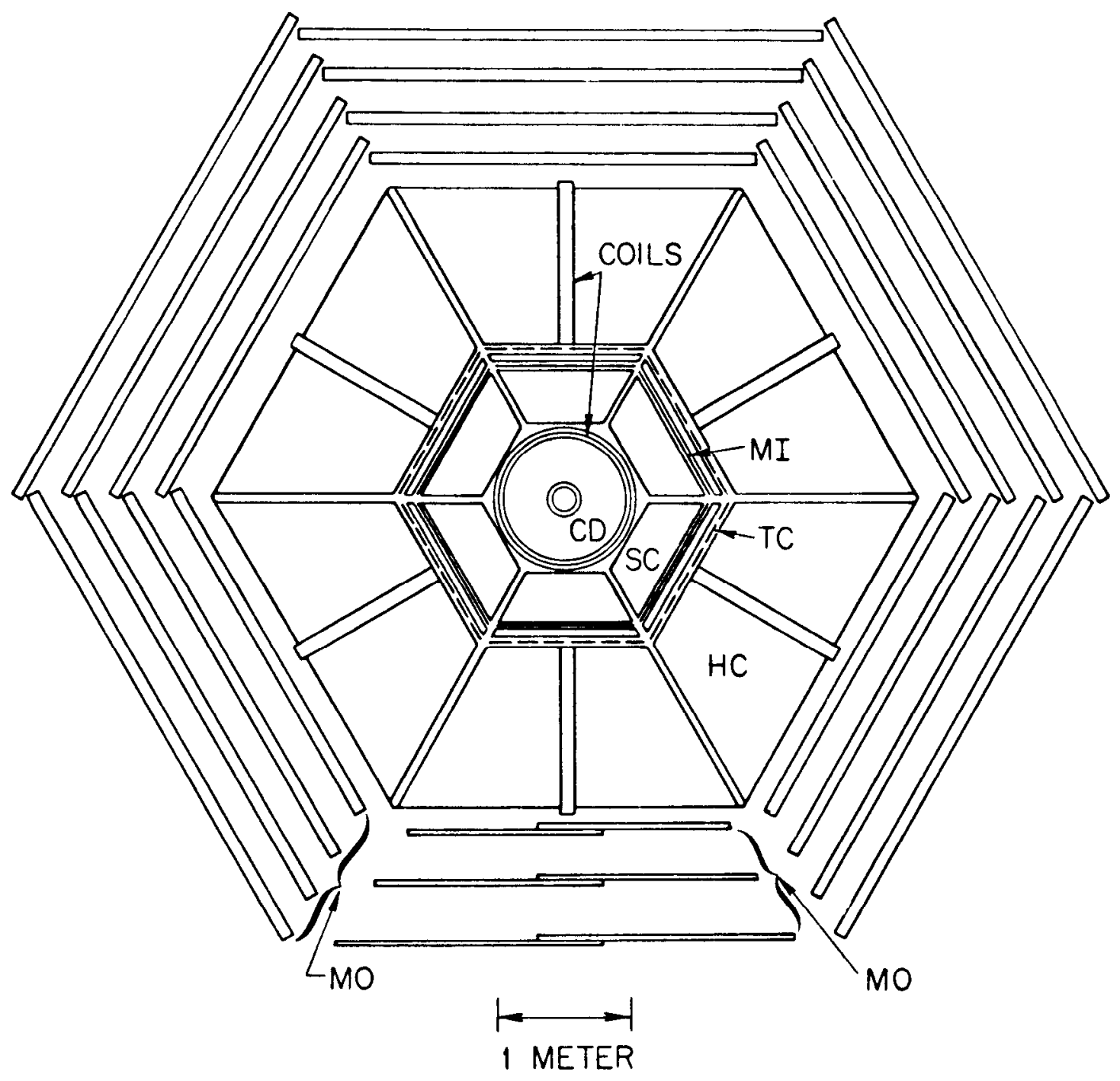

Figure 2-1: End view of the MAC detector. $C D=$ central drift chamber; $\mathrm{SC}=$ electromagnetic shower chamber; $\mathrm{TC}=$ plastic scintillator trigger counters; $\mathbf{H C}=$ hadron calorimeter; $\mathbf{M I}=$ inner muon drift chamber layer; $\mathrm{MO}=$ outer muon drift chamber; $\mathrm{EC}=$ endcap shower chamber/calorimeter; coils $=$ inner coil is solenoid, outer coil is toroid. 


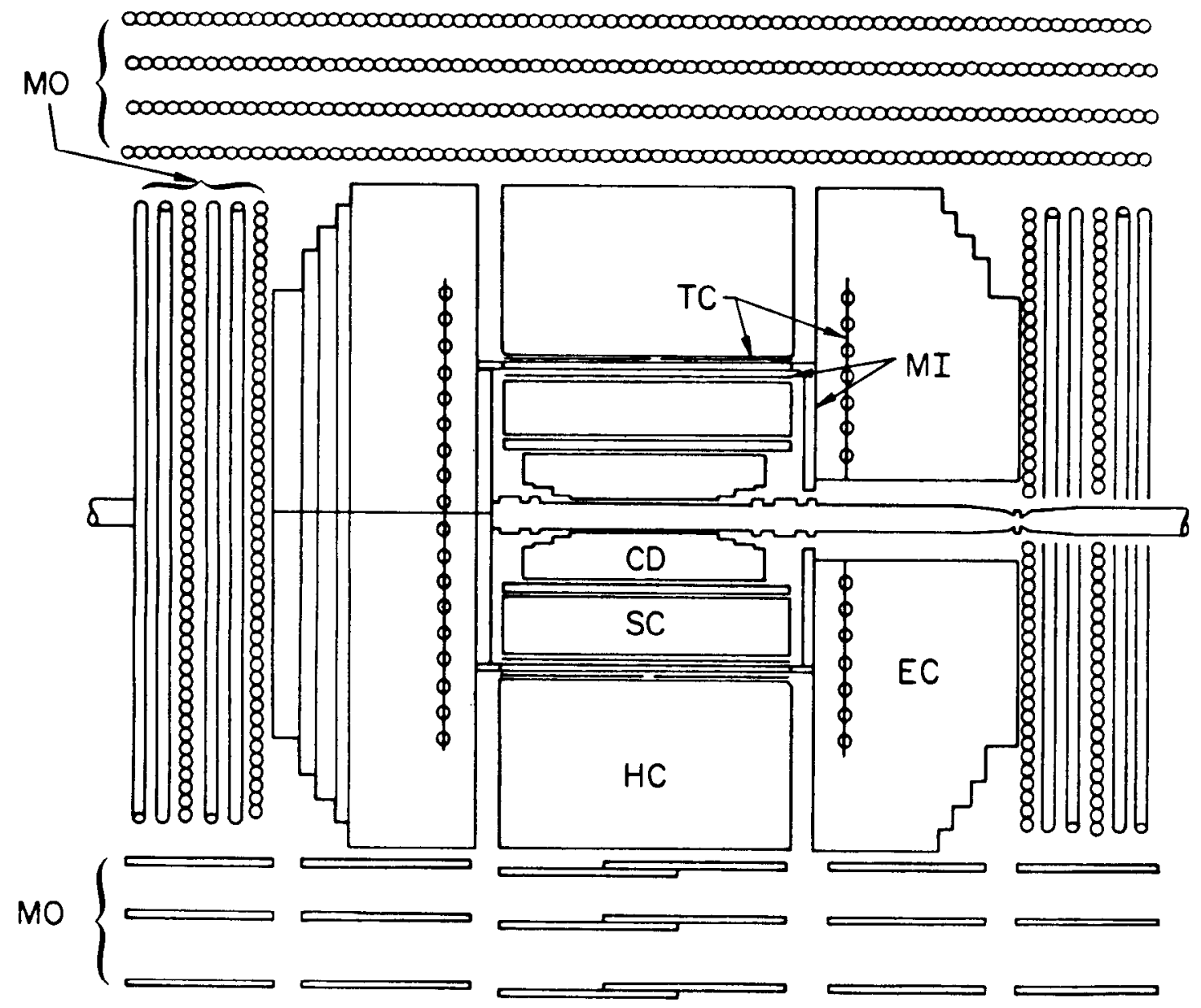

Figure 2-2: Side view of the MAC detector. $\mathrm{CD}=$ central drift chamber; $\mathrm{SC}=$ electromagnetic shower chamber; $\mathrm{TC}=$ plastic scintillator trigger counters; $\mathrm{HC}=$ hadron calorimeter; $\mathrm{MI}=$ inner muon drift chamber layer; $\mathrm{MO}=$ outer muon drift chamber; $\mathrm{EC}=$ endcap shower chamber/calorimeter; coils $=$ inner coil is solenoid, outer coil is toroid. 


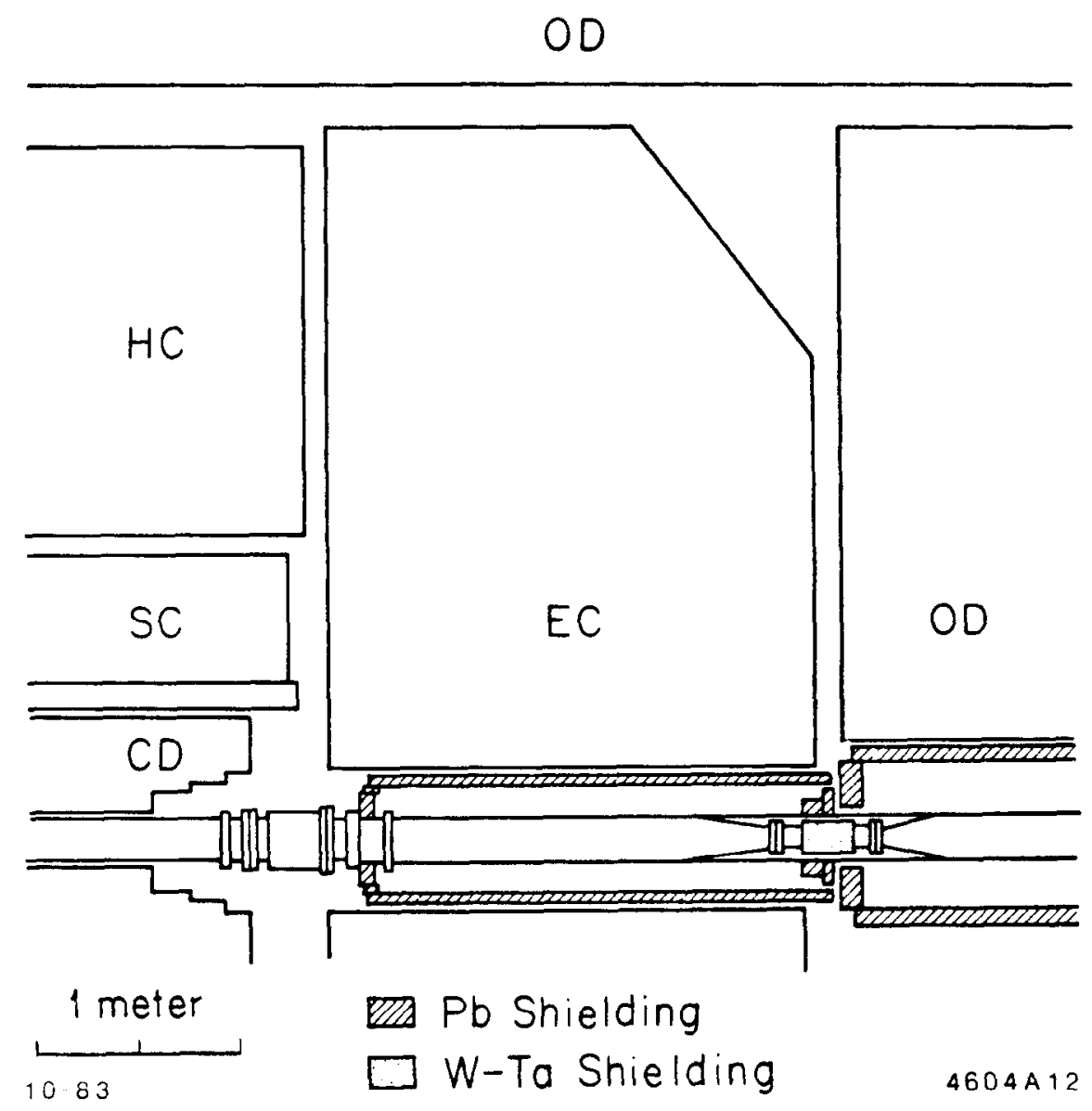

Figure 2 3: The MAC detector in the PEP storage ring. 
The blotch events are kept to a low level by a tungsten-tantalum mask inside the beam pipe and lead shielding around sections of the beam pipe.

Before describing the specifics of the MAC detector a brief explanation of the particle detection theory of each of the MAC detector components will be given.

\subsection{DRIFT CHAMBERS}

A drift chamber ${ }^{2}$ is a device used to measure the trajectories of charged particles, usually in a magnetic field. The chamber usually has thousands of "sense" wires arranged in "cells" all in a suitable gas mixture ( in MAC, similar to that of the calorimeters). There is as little material or absorber in the drift chamber as possible. The secondary ionization, avalanche, and ion drift occurs as in a proportional tube as explained in section 2.3. In a drift chamber, however, it is not the ion pulse magnitude that is of importance but rather the arrival of the electron pulse relative to a reference time. If the field shape around the wires is known (usually through the use of field shaping wires) then the drift velocity of the electrons is known. Thus the distance between the initial ionization trail and the anode can be found. This says that the charged particle passed through the drift cell tangent to an imaginary cylinder centered on the drift wire of radius:

$$
\text { distance of particle trajectory to anode }=\frac{\text { arrival time }- \text { ref. time }}{\text { drift velocity }}
$$

By having several layers of wires and not arranging the layers parallel to each other ("stereo views"), a 3-dimensional trajectory can be found by computing the best fit curve tangent through all the imaginary drift cylinders.

\subsection{The MaC Central Drift Chamber}

Before reaching the drift chamber a particle must pass through a $1.65 \mathrm{~mm}$ (65 


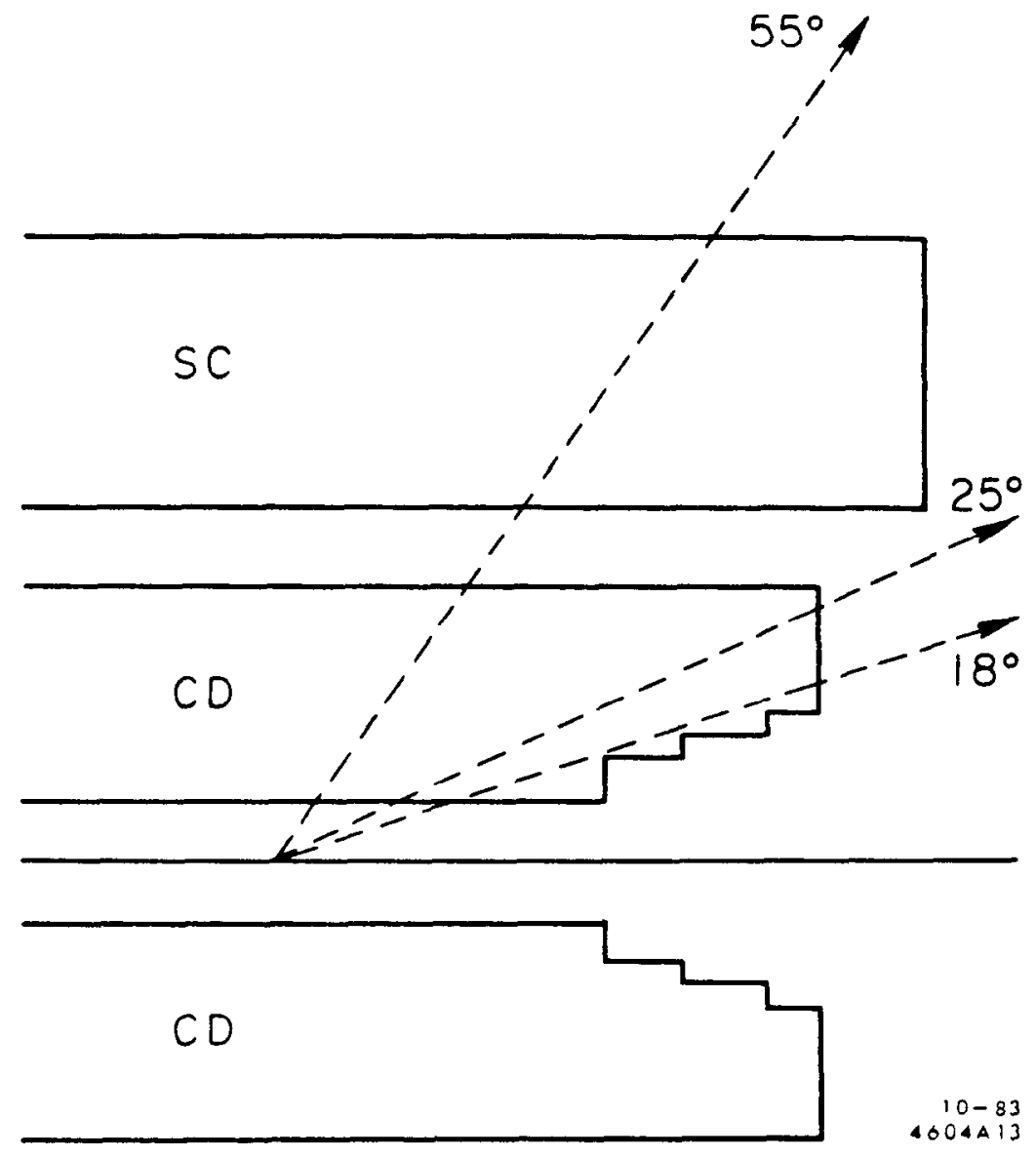

Figure 2 4: The central drift chamber with rays drawn showing the important angles in this experiment. 
mil) aluminum (.02 radiation lengths) vacuum pipe. The radius of the vacuum pipe is $4.1 \mathrm{~cm}$. The cylindrical drift chamber has 833 drift cells running the length of the chamber in ten concentric layers all in a common gas volume. The diameter is about $100 \mathrm{~cm}$ and the length is about $200 \mathrm{~cm}$. The sense wire of the innermost layer is at a radius of $12 \mathrm{~cm}$ and the outermost sense wire is at a radius of $45 \mathrm{~cm}$. The first two layers are only $\approx 60 \%$ the length of the outer six layers and the third and fourth layers are only $\approx 75 \%$ and $\approx 80 \%$, respectively. The staggered in ner four layers are referred to as the "staircase". The minimum angle between the beam line and a line going through the inner five layers is $18^{\circ}$ which is the minimum angle at which a trajectory, or "track" may be reconstructed. The minimum angle at which all ten layers are subtended is $25^{\circ}$. Figure 2-4 shows how various polar angles appear in the drift chamber. In order to measure axial positions three of the layers are $+3^{\circ}$ from axial and three layers are $-3^{\circ}$ from axial. The arrangement of layers from inner to outer, measured from axial is; $0^{\circ},-3^{\circ},+3^{\circ}, 0^{\circ},-3^{\circ},+3^{\circ}, 0^{\circ},-3^{\circ},+3^{\circ}, 0^{\circ}$. Each drift cell contains two sense wires ( $50 \mu m$ diameter) connected to differential electronics so that there is no leftright ambiguity in the drift distance. The first three layers are equipped with "double hit" electronics for better resolving of large multiplicity events. The position resolution includes effects from the position error in the holes through which wires are strung $(50 \mu \mathrm{m})$, the error in the tilt of the sleeve which holds the wire in the hole $(<40 \mu m)$, the sag in the wire due to gravity $(<50 \mu m)$, changes in the electro-static field due to the particle itself, and non-uniformities in the electro-static field due to boundary conditions. The final position resolution or point setting accuracy is $200 \mu \mathrm{m}$. With the $5.7 \mathrm{kGauss}$ axial field the momentum 
resolution is $\frac{\Delta p}{p}=.065 p(\mathrm{GeV})$. The resolution in the polar angle is $\delta \cot \theta=.008$ and for azimuthal angles $\delta \phi=0.2^{\circ}$. A system for measuring $\frac{d E}{d x}$ has also been installed. Outside the chamber is an aluminum solenoid coil which is wound in two layers totalling $18 \mathrm{~cm}$ in depth and $232 \mathrm{~cm}$ in length. The flux return is the inner five layers of the endcaps and the inner three layers of the hadron calorimeter.

\subsection{CALORIMETRY}

A calorimeter is an energy measuring device. A calorimetric detector is constructed to measure both the magnitude and direction of the energy loss of particles passing through it. It is desirable to have the calorimetry cover as much solid angle as possible and to be of sufficient thickness in order to obtain a complete as possible knowledge of the energy flow of an event. However an occasional hadron will "punch-through" the hadron calorimeter which results in not all the energy of the particle being deposited in the detector. Also muons of sufficient energy ( $>2 \mathrm{GeV}$ in $\mathrm{MAC}$ ) will pass through the detector and on to the outside world. Of course neutrinos will pass through the detector without interacting.

A typical calorimeter has alternating layers of "absorber" and "detector". The absorber (lead or iron for MAC) will cause a particle passing through it to lose energy ("shower") via electromagnetic (ionization) or nuclear (fission) interactions. The shower propagates through the absorber and it is mostly electrons which finally enter the detector layer (proportional wire chambers for MAC). The detector layer will yield information about the location and magnitude of 
the particle(s) causing the ionization. The data from the layers over the entire detector gives a picture of the energy flow of the event.

\section{Proportional Wire Chambers}

Proportional wire chambers ${ }^{3-4}$ are devices which provide a signal proportional to the amount of ionization in them in addition to providing the longitudinal position of the shower. In the MAC shower chamber, each anode wire is stretched in its own 3-sided aluminum extrusion cathode (a "tube" about $2 \mathrm{~cm}$ across and $1 \mathrm{~cm}$ deep) with lead absorber above and below. A gas mixture of $80 \% \mathrm{Ar}$ and $20 \% \mathrm{CH}_{4}$ flows through the chamber at atmospheric pressure. Noble gases are used in proportional chambers because it is easier to ionize a noble gas than a polyatomic molecule because of the latter's many non-dissipative modes. The reason for the presence of a polyatomic gas is explained below.

Electrons in the absorber freed by the traversing particle enter the tube and cause ionization. The electrons just freed in the tube will accelerate toward the anode wire. The electric field around the wire goes like $\sim 1 / r$ so most of the energy gained by the electron occurs when it is very close to the anode. While drifting toward the anode the electron will collide with gas atoms, but not until the electrons are close to the anode will they have enough energy to cause much ionization. This is because the energy gained between collisions is greater than the ionization energy only in the region close to the wire. The electrons gain energy quickly near the wire and each free electron will cause further ionization resulting in an "avalanche" of free electrons just before their collection by the anode. The collection time of the electrons by the anode wire is very short and the 
pulse induced is not very large because of the small distance between collisions, and therefore small energy gain by the electrons. The positive ions created by ionization move much more slowly than the electrons due to their much greater mass. Since the positive ions travel slowly through almost the entire potential difference to reach the cathode they induce a longer and larger pulse on the anode. When the ions get near the cathode they can extract electrons from the chamber surface releasing a photon (or another electron via secondary emission) as they neutralize. The photon can cause further ionization causing the tube to go into a continuous discharge mode. The presence of a polyatomic gas will absorb these extra photons, "quenching" the avalanche.

Photons are also produced in the ionizing collisions between electrons and atoms. Some of the photons have enough energy to free more electrons via the photoelectric effect. Let the number of electrons in the initial avalanche be $n$. The number of photoelectrons capable of producing further ionization is proportional to $n$, say $n \gamma$, with $\gamma \ll 1$ so that $n \gamma<1$. We then can write an "infinite" series for the number of electrons produced:

$$
\begin{aligned}
\text { number of electrons } & =n+(n \gamma) n+(n \gamma)^{2} n+\cdots \\
& =\frac{n}{1-n \gamma}
\end{aligned}
$$

This equation says that the number of electrons and therefore the number of ions and hence the induced voltage is proportional to the initial ionization in the chamber.

For electromagnetic showers the energy resolution is limited by fluctuations of ionization in the sampling layers (Landau fluctuations in the energy of showering electrons) and by the fact that knock-on electrons scattered by a large angle in 
gas can produce much more ionization than if it had gone straight through. ${ }^{5-6}$

Hadronic showers are much more complicated than electromagnetic showers. In hadronic showers the electromagnetic component comes from $\pi^{\mathbf{0}}$ production. The nuclear component comes from the slow moving, heavy ionizing particles produced in the hadron-nucleon strong interaction. Some of the particles produced will be protons and neutrons kicked out from the nucleus. The binding energy of the nucleons is undetected as are any neutrinos produced. Thus, the energy resolution of a hadronic shower is intrinsically worse than that of an electromagnetic shower because of the possibility of energy fluctuations due to nuclear binding effects and neutrino production.

\subsection{The MaC Electromagnetic Shower Chamber}

Outside the solenoid coil is a hexagonal electromagnetic shower chamber. Each sextant has 32 alternating layers of lead $(83 \% \mathrm{~Pb}, 12 \% \mathrm{Sb}, 5 \% \mathrm{Sn})$ and proportional chamber. The total thickness of the chamber corresponds to 16 radiation lengths. (A radiation length is the distance over which an electron's

energy is reduced by a factor $\frac{1}{e}$.) The proportional chambers are made with aluminum extrusions. Each channel in the extrusion (cathode) is about $2 \mathrm{~cm}$ across and $1 \mathrm{~cm}$ deep with a $50 \mu \mathrm{m}$ anode wire running the length of the extrusion. The chamber is divided into 192 azimuthal segments and 3 layers in depth by ganging different proportional chambers together. Altogether, then, the shower chamber has $192 \times 3 \times 2=1152$ electronics channels or wire groups read out.

To determine axial position of a shower "charge division" is used, that is, the Voltage pulse-height induced at each end of the wire group is measured after a 
Voltage pulse-height induced at each end of the wire group is measured after a low impedance $(\approx 7 \Omega)$ pre-amplifier. The longitudinal position along of a shower along a wire group, $z$, determined from charge division can be written as:

$$
z=\frac{\left(\frac{R_{\text {preemp }}}{R_{\text {wiregroup }}}+\frac{1}{2}\right) V_{\text {end1 }}-\left(\frac{R_{\text {presmp }}}{R_{\text {wiregroup }}}+\frac{1}{2}\right) V_{\text {end2 }}}{V_{\text {end1 }}+V_{\text {end2 }}} \cdot l_{\text {wire }}
$$

where the $V$ 's are the digitized induced voltages on either end of the wire group, the $R$ 's are resistances, and $l_{\text {wire }}$ is the length of the wire. The intrinsic position resolution is limited by several effects. A typical shower will deposit charge at different $z$-positions as it passes through the proportional chambers at an angle. Since several chambers contribute to a wire group the $z$-position of a wire group is the charge-weighted average $z$ of the individual wires in the wire group. The uncertainty in the position resolution is also affected by the noise in the wire and the pre-amplifier, the digitization error, the non-linearity of the electronics, and the uncertainty in the effective input impedance of the external circuitry. The noise and non-linearity effects are minimized by the circuit design. The relative error due to digitization is small for heavily showering particles like electrons but is significant $(\approx 2 \%)$ for minimum ionizing particles like $\mu$ 's. The effective input impedance of the external circuity is affected by such things as the chamber design and the sample timing (the time when the pulse height is read out) of the electronics.

Several effects make the chamber performance depart from less than ideal behavior. Small dead spaces exist where the sextants meet, where an integer number of extrusions don't fit into a layer, and in proportional tubes with broken wires (this last effect is greatly reduced because of the ganging of about ten wires 
in a wire group for each read out electronics channel). At the less than $0.2 \%$ level per channel there is electrical cross-talk between the 32 channels on a preamplifier board. The magnitude of cross-talk varies from channel to channel. The cross-talk adds both spurious energy and misassigns the spatial position of the apparent additional energy. This last effect is the most serious and results in a systematic distortion of longitudinal position measurements. The energy calibration and resolution of the shower chamber is measured with Bhabha events produced at a known energy. The energy resolution is about $20 \% / \sqrt{\mathrm{E}(\mathrm{GeV})}$. By comparing extrapolated tracks from the central drift chamber with shower centroids the directional resolution is measured. The angular resolution is $\Delta \phi=$ $0.8^{\circ}(\approx 0.4$ times the wire group segmentation $)$ and $\Delta \theta=1.3^{\circ}(\approx 1 \%$ fo the wire length).

\subsection{The SCIN TILlation COUNTER SYSTEM}

The scintillation counter system is used for time-of-flight measurements and triggering. The time-of-flight information is used to reject cosmic rays. The role of the scintillation counters in the trigger system is explained in the next chapter.

Outside of the shower chamber (central and endcap sections) is a single layer of plastic scintillation counters. There are 144 scintillation counters; 6 on outer face the shower chamber sextants and 36 in each endcap. Briefly, a scintillation counter works in the following way; a charged particle passing through the plastic causes ionization. The ionization energy eventually reaches an "impurity center" which will convert the energy to a visible photon. One end of the plastic is optically connected to a photomultiplier tube which amplifies the photon signal. 
The photon reaches the photomultiplier by total internal reflection within the plastic. The timing resolution of the MAC scintillation counters is about $1 \mathrm{nsec}$.

\subsection{ThE MAC Hadron Calorimeter}

Outside the trigger counter layer is the hadron calorimeter. The hadron calorimeter is similar in structure to the shower chamber but instead of using lead as an absorber 1-inch thick steel plates are used. The same kind of proportional wire chamber (PWC) is used in the hadron calorimeter as in the shower chamber. The segmentation and readout is also the same as in the shower chamber. There are 24 steel plates interleaved with proportional chamber followed by three 4inch thick steel plates for muon tagging. This thickness of iron corresponds to 5.4 absorption lengths. (An absorption length, or geometrical mean free path, is simply $\frac{1}{N \sigma}$ where $N$ is the number density of the absorber atoms and $\sigma$ is the geometrical cross section, $\pi r^{2},\left(r \approx A^{\frac{1}{3}}\right)$ of the atoms in the absorber.) The central section is $230 \mathrm{~cm}$ long with an inner diameter (minimum distance between opposing sextants) of $114 \mathrm{~cm}$ and an outer diameter of $257 \mathrm{~cm}$.

The calorimetry is extended to small angles with two endcaps. In the endcaps the steel plate absorbers are perpendicular to the beam line. In each endcap there are 28 1-inch thick layers of steel and two 4-inch muon tagging layers each with their associated PWC. The chambers in the first 9 gaps (16 radiation lengths) have finer segmentation than the rest of the endcap and act as shower chambers. Each chamber covers $30^{\circ}$ azimuth. The anode wires run parallel to the inner and outer edges of the hexagonal endcap and are read out in four radial sections. The cathode strips are grouped into three $10^{\circ}$ azimuthal sections and 
are also read out. For read out purposes the endcap is divided into four layers in depth. Each segment of the chambers is about $5^{\circ}$ in azimuth and $5^{\circ}$ in the polar direction. The steel in the central and endcap sections is magnetized by toroid coils which provide an azimuthal field of $17.5 \mathrm{kGauss}$. The calorimeter was designed to provide a low reluctance path for the solenoid coil flux return. A high reluctance air gap prevents the calorimeter azimuthal magnetic field from entering the flux return layers. The calibration of the hadron calorimeter is measured using multi-hadron events where nearly all the energy is detected. The calibration is checked with cosmic rays. The energy resolution for the central hadron calorimeter is $50 \% / \sqrt{\mathrm{E}(\mathrm{GeV})}$ and for the endcaps is $100 \% / \sqrt{\mathrm{E}(\mathrm{GeV})}$.

The angular resolution for the central calorimeter is $450 \mathrm{mrad} / \sqrt{\mathrm{E}^{3 / 4}}$.

\subsection{The MuON Tracking System}

One of the features of the MAC detector is the ability to do muon spectroscopy. Muons with a momentum $>2 \mathrm{GeV} / \mathrm{c}$ will penetrate the calorimeter steel and enter the outer drift system.

The muon tracking system consists of two parts, an inner part consisting of one central and three endcap planes of drift chamber in front of the hadron calorimeter faces, and an outer part consisting of four layers of drift tubes surrounding the calorimeter sextants (the layers on the underside are planar due to the structural supports) and six layers covering the outer endcaps faces. The endcap drift tubes have two layers which are horizontal and two layers each at $60^{\circ}$ and $120^{\circ}$ to the horizontal, respectively. The aluminum drift tubes are $10 \mathrm{~cm}$ in diameter and contain one wire. The azimuthal magnetic field in the hadron 
calorimeter causes a change in the polar angle of a charged particle and hence allows a determination of axial position by the outer tubes. The spatial resolution is about $1 \mathrm{~cm}$ limited mainly by multiple scattering in the hadron calorimeter. Combined with other detector data the momentum resolution of the outer drift system is about $30 \%$.

\subsection{RUNNING THE EXPERIMENT}

The MAC detector uses a computerized monitor system to continually check power supply voltages, temperatures, etc. throughout the detector. Checks are made on the hardware operations and also for oxygen content of the PWC gas and drift chamber gas. Under most conditions only one person is needed to run the experiment. The shift person is required to see that data logging proceeds

smoothly, that is, begin and end runs and respond to warning messages from the monitor system. The monitor system communicates with the shift person through a computer terminal.

\subsection{Comparison with Other Detectors}

No other detector has as much coverage with calorimetry as the MAC detector. The price for this coverage is a somewhat small inner drift chamber. The use of inner detectors varys in sophistication from none at all to the very sophisticated Time-Projection Chamber. In terms of calorimetry the MAC calorimetry relies on proportional wire chamber-absorber sandwiches while other detectors may use plastic scintillator-absorber sandwiches. Some detectors have no calorimetry but have particle identification with time-of-flight systems or $\check{C}$ erenkov counters. 

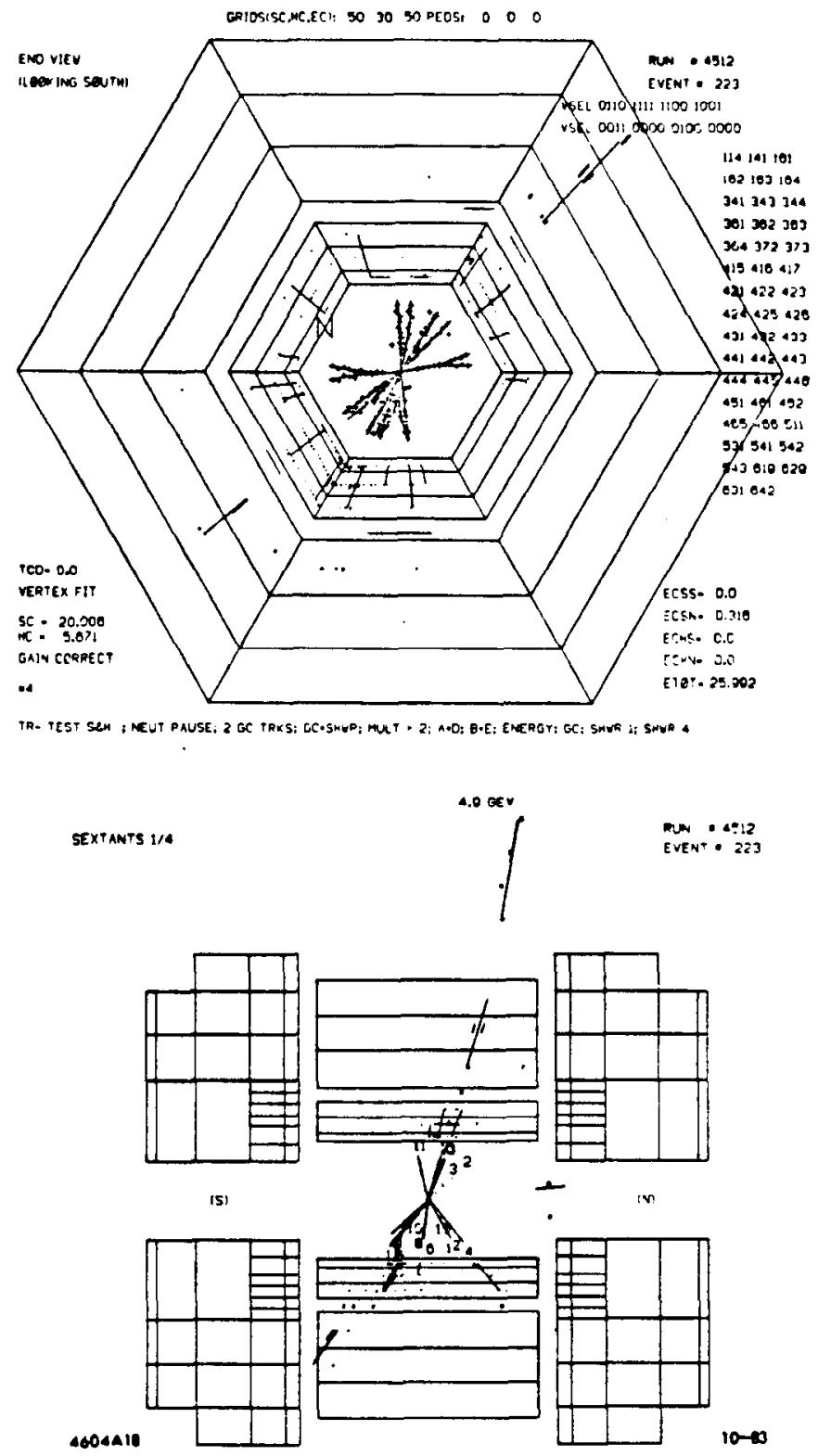

Figure 2-5: A typical multi-hadron event in the MAC detector. The top picture shows an end view of the detector with the beam axis perpendicular to the page. The bottom picture is a side view of the same event. Further explanation is given in the text. 


\subsection{A Typical Multi-Hadron Event in MAC}

Fig 2-5 shows a typical multi-hadron event in the MAC detector. Referring to the end view of the figure, the hits in the central drift chamber (" + ") are clearly shown along with computer drawn particle trajectories. The pulse heights in the shower chamber and hadron calorimeter are shown along with computer tracking drawn through the showers. The scintillator hits are denoted by thin lines between the shower chamber and hadron calorimeter corresponding to their position in the detector. In the side view one of the particles goes through the calorimetry and emerges into the outer drift system. The ${ }^{4 * \pi}$ in the side view correspond to hits in the inner muon drift system. The particle in the outer drift chamber in this case is likely a $\mu$ from the leptonic decay of a quark. 


\section{REFERENCES}

1. Ford, W. T., "The MAC Calorimeters and Applications", in Proceedings of the International Conference on Instrumentation for Colliding Beam Physics, SLAC Report-250,174 (1982).

2. Charpak, G. and F. Sauli, Nucl. Instr. Meth., 162, 405 (1979).

3. Charpak, G. and F. Sauli, Nucl. Instr. Meth., 162, 405 (1978).

4. Rossi, B., High Energy Particles, 102-104, (Prentice-Hall, N.J., 1852).

5. Ritson, D. M., "Instrumentation", in Proceedings of the SLAC Summer Institute, 177 (1980).

6. Gordon, H, A. and S. D. Smith, "Sampling Calorimeters in High Energy Physics", in Proceedings of the SLAC Summer Institute, 241 (1980). 


\section{Whapter 3}

\section{Data Acquisition System}

This chapter will describe the flow of data from the MAC detector and the MAC analysis system.

The data flow starts at the hardware trigger. A hardware trigger occurs when certain conditions of energy deposition, scintillator hits, etc. are met. A hardware trigger must be in time with the beam crossing. After passing the hardware trigger the "events" are put through a software trigger. The software trigger is a computer generated trigger which is more sophisticated than the hardware trigger. Events passing the software trigger are stored on disk or tape in preparation for a much more detailed software analysis. Figure 3-1 shows the data flow for the MAC experiment. The rest of this chapter will describe in detail the various components of the MAC data acquisition system.

\subsection{HARDWARE TRIgGer SYSTEM}

PEP normally runs with three bunches of electrons and positrons corresponding to a beam crossing rate of $409 \mathrm{kHz}$ or a beam crossing every $2.4 \mu \mathrm{sec}$.

The MAC detector has a VAX $11 / 780$ computer which does preliminary data manipulation and storage. The VAX must be alerted to the fact that an event has occured. Signals from various parts of the detector form hardware triggers 


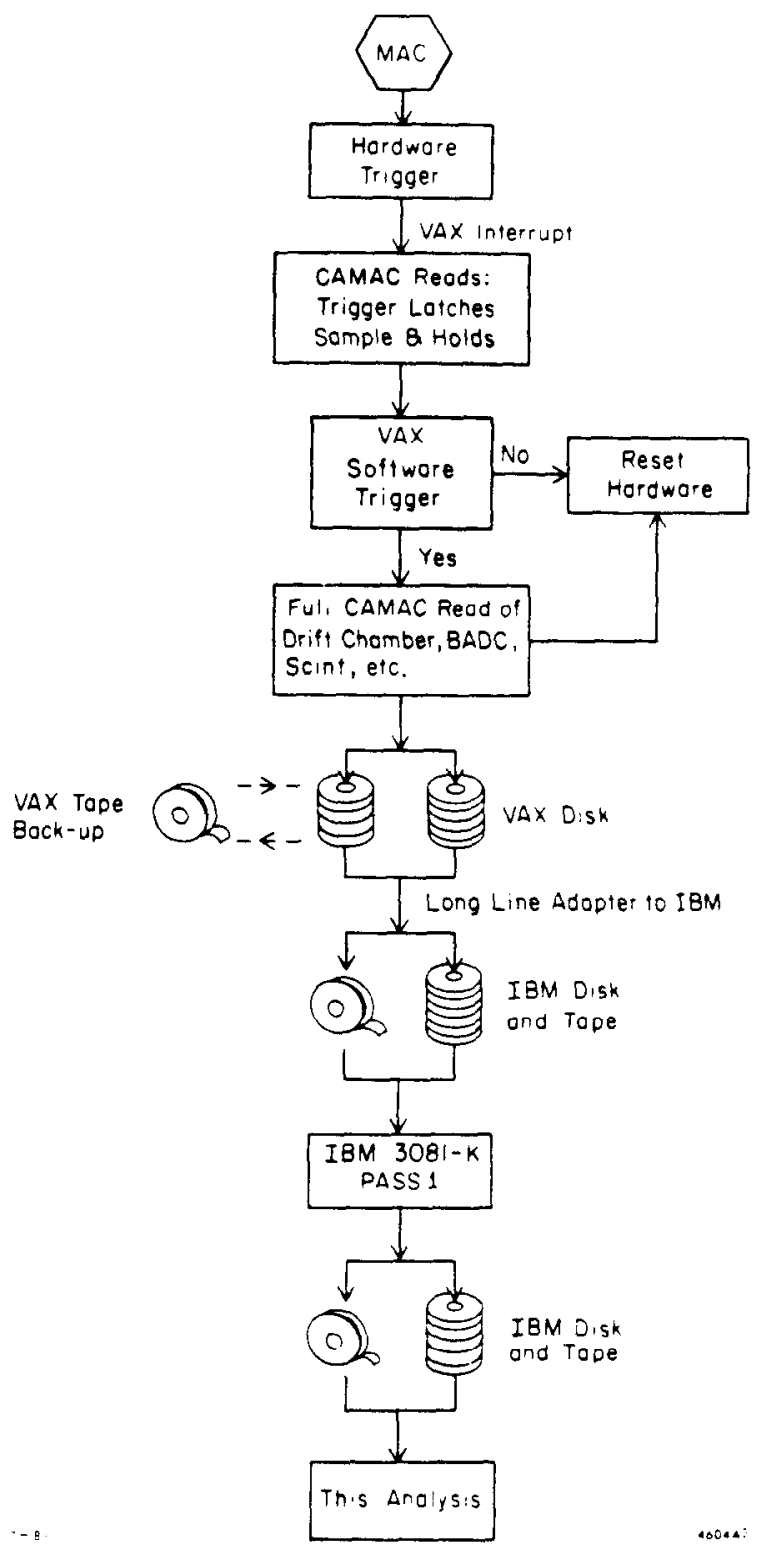

Figure 3-1: Data flow using the MAC detector. 
that are used to alert the VAX to an event. If any of the hardware triggers is true a $1 \mu \mathrm{sec}$ pulse is put out which inhibits the resetting of electronics until the VAX has recorded the detector information. There are two types of hardware triggers, the Fast and Slow which will be described in turn.

\section{The Fast Trigger}

The Fast trigger consists of coincidences between scintillation counter signals. These coincidences are formed $\approx 200 \mathrm{nsec}$ after the beam crossing. The geometry of the scintillation counters has been described in the previous chapter. The output of each photomultiplier tube is sent to an Analog-to-Digital Converter (ADC) channel and discriminator channel. The discriminators are gated on for $40 \mathrm{nsec}$ around the beam crossing. The discriminator produces two outputs; one for the "trigger bus" and the other which is used in combination with other channels to form the Fast trigger. Signals from the discriminators (NIM pulses) are logically ORed with other signals from a similar part of the detector. The signals formed are the following:

CS (A-F), 6 pulses from the OR of the 12 counters in each sextant;

NECQ (NQ1-4), 4 pulses from the OR of the 9 counters in each quadrant;

SECQ (SQ1-4), 4 pulses from the OR of the 9 counters in each quadrant;

NEC, 1 pulse from the OR of the NEQ;

SEC, 1 pulse from the OR of the SEQ,

where $N=$ north,$S=$ south, $E C=$ endcap,$C S=$ central sextant, and $Q=$ quadrant. 
These signals are combined to form 3 types of Fast triggers. The first type involves coincidences between opposing central sextants, specifically; $A^{*} D, B^{*} E$, or $\mathrm{C}^{*} \mathrm{~F}$ where * means logical AND. Second, there are coincidences between opposing endcap quadrants; NQ1*SQ3, NQ2*SQ4, NQ3*SQ1, and NQ4*SQ2. Finally, a trigger can be made if there are three or more of the six sextant or two endcap sum pulses in coincidence.

The Slow Trigger

The second type of hardware trigger used in MAC is called the Slow trigger. Slow triggers are more complex than Fast triggers and require $\sim \mu$ sec to be formed. Since most of the data logging electronics is reset $\approx 0.7-1.8 \mu \mathrm{sec}$ after the beam crossing $(\approx 0.7 \mu \mathrm{sec}$ till the reset of the scintillation counter ADC and TDC units, $\approx 1.6 \mu \mathrm{sec}$ till the reset of drift, trigger bus, and energy systems), a Slow trigger must be preceded by an inhibit (pause) which prevents the resetting of electronics until a Slow trigger is made. Also the luminosity monitor is gated off during a system inhibit so that it records only when the detector is "alive". The typical dead time for a run is $5-10 \%$.

There are three types of pauses; Scintillator, Neutral, and Central Drift. The Scintillation pause fires when any scintillator is hit. The pause is $2.4 \mu \mathrm{sec}$ long and runs at $200-1000 \mathrm{~Hz}$. A Neutral pause will occur if no scintillator fires and if $\geq 2$ out of the 8 energy signals ( 6 central shower, 1 each endcap, and 1 central calorimeter) are above threshold and in a $250 \mathrm{nsec}$ gate with respect to a fixed delay ( $1200 \mathrm{nsec}$ ) after the beam crossing. This pause is $2.4 \mu \mathrm{sec}$ long and runs at about $3 \mathrm{~Hz}$. Finally there is a Central Drift pause which requires 2 Large Angle 
Central Drift wedges (a wedge covers $20^{\circ}$ azimuth and fires if there are more than 2 hits in both the inner 5 layers and outer 5 layers) with $>90^{\circ}$ between them. This pause runs at $\approx 30-50 \mathrm{~Hz}$.

The Slow triggers are based on information from central drift wedges, energy sums, and trigger counter circuits. There are 36 central drift wedges each covering $20^{\circ}$ in azimuth. A Small Angle Drift wedge is made if there are $\geq \mathbf{3}$ hits in the inner 5 layers and $<3$ hits in the outer 5 layers. A Large Angle Drift wedge is made if there are $\geq 3$ hits in the inner 5 layers and outer 5 layers. Central scintillation wedges are divided into the same azimuthal sectors as the Central Drift wedges and one on each end (1 North, 1 South), totalling 36 signals. The Large Angle Hadron wedges are also divided into north and south $20^{\circ}$ azimuthal components, 18 North Hadron Sextant wedges from the central calorimeter and north endcap, and similarly for the 18 South Hadron Sextant wedges. Also there are 8 (4 north, 4 south) Endcap Scintillation wedges and Endcap Hadron wedges.

One of the Slow triggers is the muon track processor, or Grand Crate, which uses the wedge signals just described. Grand Crate tracks are formed from coincidences of central drift wedges and the corresponding Scintillation and Hadron wedges. There are 44 possible Grand Crate tracks (18 north-central + 18 southcentral +4 north-endcap +4 south-endcap). 
A Large Angle Grand Crate track ( 36 possible) is made from a coincidence of the following:

18 Large Angle Drift wedges

AND

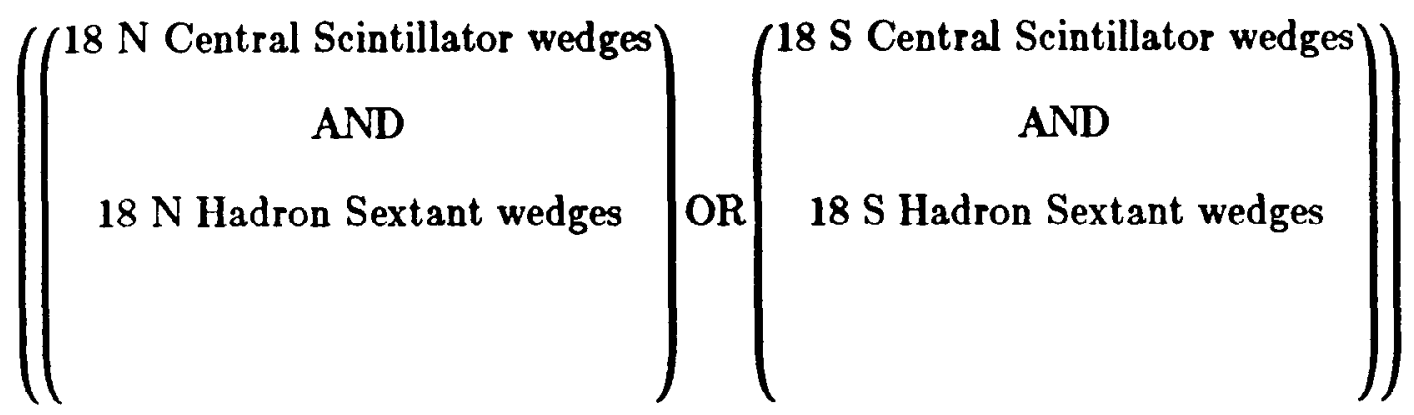

A Small Angle Grand Crate track (8 possible) is made from:

18 Large OR Small Angle Drift wedges

AND

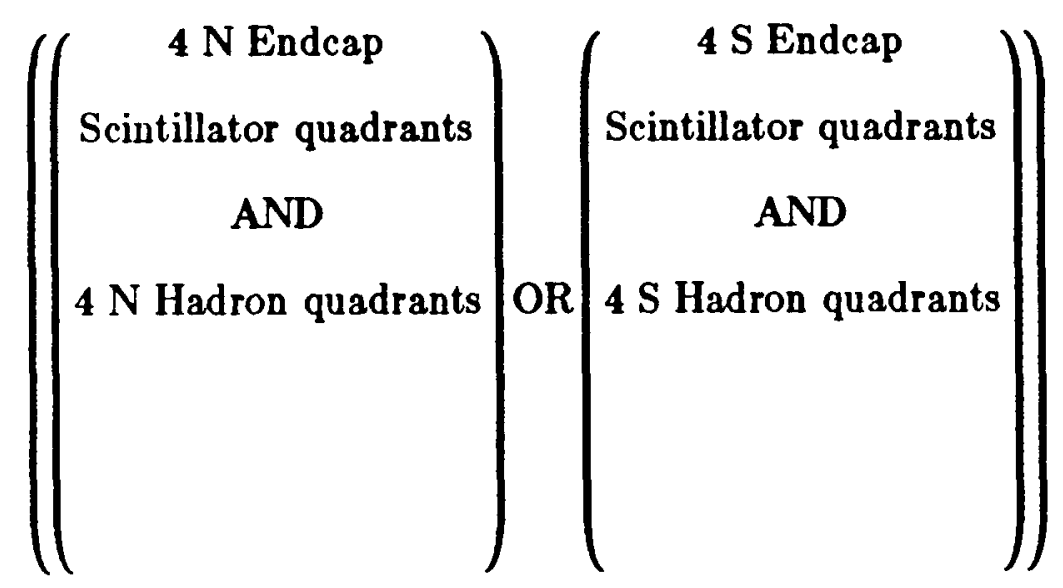

A Grand Crate trigger is made from the OR of the 44 Grand Crate tracks. A similar trigger is the Grand Crate* Shower trigger is a coincidence of three adjacent Grand Crate wedge signals with the corresponding shower chamber pause. 
Another Slow trigger is the CD*Show2 which is made from Large Angle Central Drift quadrants and shower energy sums (6 central, 6 endcap). A Large Angle Central Drift quadrant is a coincidence between 5 or 6 Large Angle Central Drift wedges. The quadrants are overlapping, 3 covering $120^{\circ}$ in azimuth each and one covering $100^{\circ}$ in azimuth. A CD*Show2 trigger is made if there are back-to-back Central Drift quadrants and $\geq 2$ shower sextants (out of 18) are above threshold.

The Energy trigger is another Slow trigger which uses 9 energy sums; the 6 central shower sums, 2 endcap sums, and a central hadron sum. The shower sums are connected to discriminators which form the Energy trigger. An Energy trigger is made form the OR of any of the 9 energy inputs.

When a Slow trigger has been made the resetting system is inhibited and the VAX will read in data from the detector. Each drift chamber cell is connected to a pre-amplifier, discriminator, and a Time-to-Voltage Converter (TVC). Each wire group in the calorimeters is connected to a pre-amplifier. The pre-amps take the raw signals from the detector and amplify and shape the signals. Signals from the pre-amplifier are sent to a Sample and Hold module (SHAM), and digitized by a "Brilliant" Analog-to-Digital Converter (BADC). Each BADC is in a CAMAC crate and the CAMAC crates are daisy-chained through their Crate Controllers. A Branch Receiver on one of the crates is connected to a Branch Driver on the System Crate which is finally connected to the VAX via a "Jorway" 411 VAXto-CAMAC Adapter. The typical hardware trigger rate is $3-5 \mathrm{~Hz}$. There are four main hardware triggers that trigger multi-hadron events. These are the Energy trigger, the CD*Show2, the Grand Crate, and the Scintillator (Fast) 
trigger. A simulation of the hardware trigger by the Monte Carlo shows that the multi-hadron events used in this analysis will trigger the apparatus $99.9 \%$ of the time.

\subsection{The MaC Online Software Filter}

The online software filter is designed to provide orthogonal triggers on which all events are tested. The software triggers (about 25 ) are made from combinations of energy sums, Grand Crate tracks, scintillation coincidences, and other quantities constructed in the hardware. The thresholds in the software trigger are tighter than at the hardware level. An event passing any of the software triggers is saved on disk and tape and later analyzed by the offline filter. A typical run lasts about an hour, takes up about 15 Mbytes of storage and contains about 12,000 events.

\subsection{OfFLine Filter}

When the VAX disks become full the data are shipped via coaxial cable to IBM disks. Further computer analysis (PASS1) is done on an IBM 3081-K in a computer routine called FSTFLT (FaST FiLTer). It takes about 30 minutes of IBM 3081-K time to perform the PASS1 analysis on a typical data run of one hour duration. The PASSI analysis reconstructs central and outer drift tracks, showers in the calorimeters, and computes a variety of useful quantities, eg. thrust angle. This program also serves as a sophisticated software filter. The events are put through a truth table or "mask set" to determine if the events are of physics interest. An event has passed a mask if it satisfies all of the up to 19 attributes of that mask. There are two levels of mask sets. Every event saved 


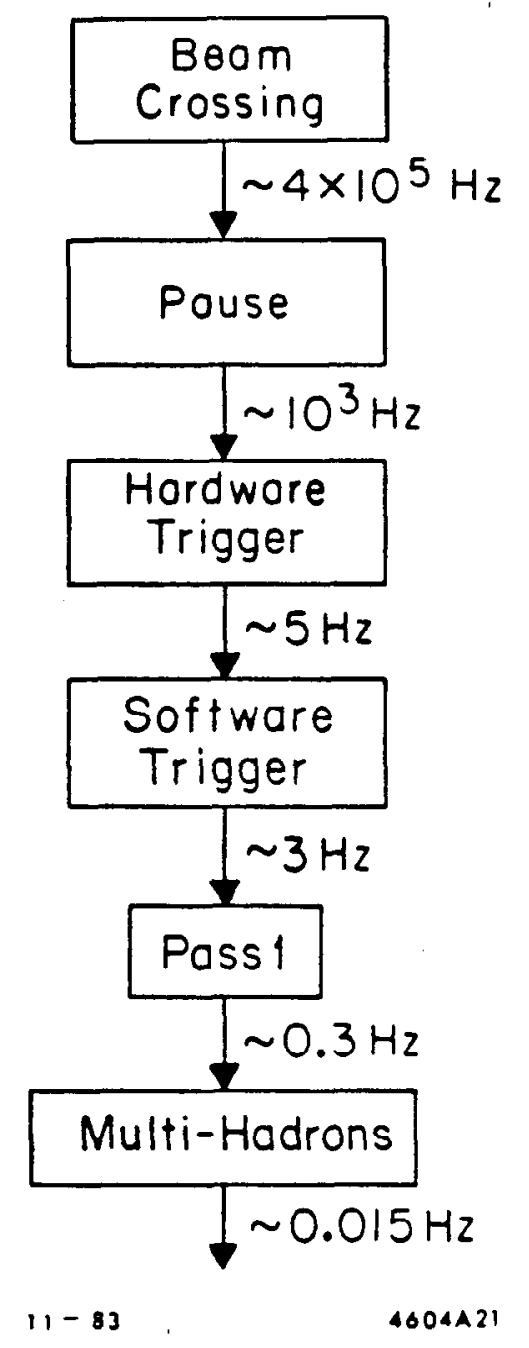

Figure 3-2: Data flow rate through the on-line and off-line filter systems. 
by the VAX is analyzed to see if it satisfies any of the lowest level masks. The purpose of the low level masks is to identify all events that could be of physics interest. Only events passing any low level mask receive further analysis. The high level masks attempt to classify the events into certain very loosely defined topologies, eg. Bhabha, hadronic, $\mu$ - or $\tau-$ pair. Events passing any high level mask are saved on disk and tape $(\approx 10 \%$ of the VAX events). Events passing the highest level masks allow are further tagged to allow a relatively pure sample of events to be quickly selected for further analysis. Figure 3-2 shows the rate of data flow through the MAC analysis system. The selection of multi-hadron events corresponds to about one event per half-billion beam crossings.

The software trigger rate has been a fairly constant $3-5 \mathrm{~Hz}$ throughout the running history but the luminosity has increased by more than a factor of ten so that there is a more efficient use of the beams in providing collisions. Most of the events saved by the final filter are two photon events and low angle Bhabha events. The main source of non-physics events in the stored data are from beamgas events. 


\section{Chapter 4}

\section{Data Analysis}

\subsection{INTRODUCTION}

In principle the measurement of the $\mathbf{R}$-value for multi-hadron production in $e^{+} e^{-}$annihilation (hereafter referred to as annihilation multi-hadron production) can be made by simply counting the total of all multi-hadron events produced, a quantity directly relatable to $R$. In practice backgrounds are present from closely related processes such as multi-hadron production from two photon-annihilation. Also the apparatus has finite acceptances and resolution, and the triggering systems are not $100 \%$ efficient. Therefore to achieve a precise determination of the R-value for annihilation multi-hadron production the acceptance phase space must be defined by cuts designed to minimize the systematic errors associated with these effects.

The requirement that an event contains several charged tracks coming from a common vertex (verticizing tracks) strongly reduces many sources of backgrounds. If more than five verticizing tracks are required virtually all the $\tau$-pair events (accounting for $\sim \sigma^{\mu \mu}$ of cross section) are eliminated while leaving about 95\% of the annihilation multi-hadron events. This cut was used in the final analysis. 
Even with the charged track cut there is a background of $2 \gamma$ multi-hadron events predominantly associated with production at low-energies and at small angles to the beam line. The criteria of total energy and "energy-imbalance" serve to differentiate $e^{+} e^{-}$annihilation multi-hadron events from the $2 \gamma$ processes.

The "energy-imbalance" is a measure of the missing momentum along the beam direction. "Energy-imbalance" is defined as:

$$
I=\frac{\mid \sum_{E_{n e r g y}\left(E_{e^{+}}-E_{e^{-}}\right) \mid}}{\sum_{\substack{E_{n i t e} \\ \text { hite }}} E_{i}}
$$

where $E_{e^{+}}\left(E_{e^{-}}\right)$is the energy readout on the end of the detector from which the positron (electron) beam is incident. $E_{e^{ \pm}}$includes the current divided energy readout from the end of the appropriate central section plus the corresponding endcap. For annihilation multi-hadron events momentum should balance, whereas for $2 \gamma$ events unobserved momentum is likely to be carried away by the $e^{+}$and $e^{-}$along the beam direction. Imbalance therefore can serve to distinguish between these two classes of events.

Another useful parameter to differentiate these two classes of events is the production-angle or "thrust-axis". For annihilation multi-hadron events the production angles of the primordial quarks should be distributed with a $1+\cos ^{2} \theta$ angular distribution in the center-of-mass frame $(\approx$ lab system for the annihilation process) and the distribution of the "axis of final state momenta flow" ("thrust axis") closely follow this distribution. For two photon events the center-of-mass system does not in general correspond to the lab system and the "thrust-axis" distribution will be peaked strongly toward small angles to the beam axis.

The energy thrust axis is defined as the direction of the unit vector, $\hat{n}$, which 
maximizes the quantity called thrust:

$$
\mathrm{T}=\frac{\max \left(\sum_{\substack{E_{\text {hite }} \\ \text { hite }}} \overrightarrow{\mathrm{E}}_{i} \cdot \hat{n}\right)}{\sum_{\substack{E_{\text {nergy }} \\ \text { hite }}} \mathrm{E}_{i}} .
$$

Figure 4-1 shows plots, for a sample events, of three-dimensional histograms obtained by plotting the number of events versus the total event energy on one axis and the energy imbalance on the other axis. The plots are divided into four angular ranges as determined from the thrust axis, $55^{\circ}<\theta_{E_{\text {thrut }}}<$ $90^{\circ}, 30^{\circ}<\theta_{E_{\text {thruat }}}<55^{\circ}, 15^{\circ}<\theta_{E_{\text {threst }}}<30^{\circ}, 0^{\circ}<\theta_{E_{\text {thrast }}}<15^{\circ}$, and are further subdivided into histograms containing events with five or more charged tracks, and with nine or more charged tracks. Inspection of the figures shows that at low angles there is no clear division between multi-hadron production and background processes such as $\tau$-decays, two-photon processes, cosmic ray events, and interactions of the beam with the shielding, etc.

Histograms $(g)$ and $(h)$ for the angular range $55^{\circ}<\theta_{E_{\text {thruot }}}<90^{\circ}$ for low (and also high) multiplicity events show a clear peak centered around a total energy of twice the beam energy along the energy axis and along the imbalance axis grouped towards low imbalances. This peak is clearly consistent with events arising from annihilation multi-hadron events, and is inconsistent with the distribution from $2 \gamma$ and other background processes that would be expected to peak at low energies and high imbalance. Again with reference to this plot an energy cut at $16 \mathrm{GeV}$ would suffice to cut out nearly all of the small residual backgrounds without seriously effecting the annihilation multi-hadron signal.

Histograms (e) and $\left(\Omega\right.$ for the angular range $30^{\circ}<\theta_{E_{\text {lhruat }}}<55^{\circ}$ still show for both high and low multiplicity clear peaks with the characteristics expected 


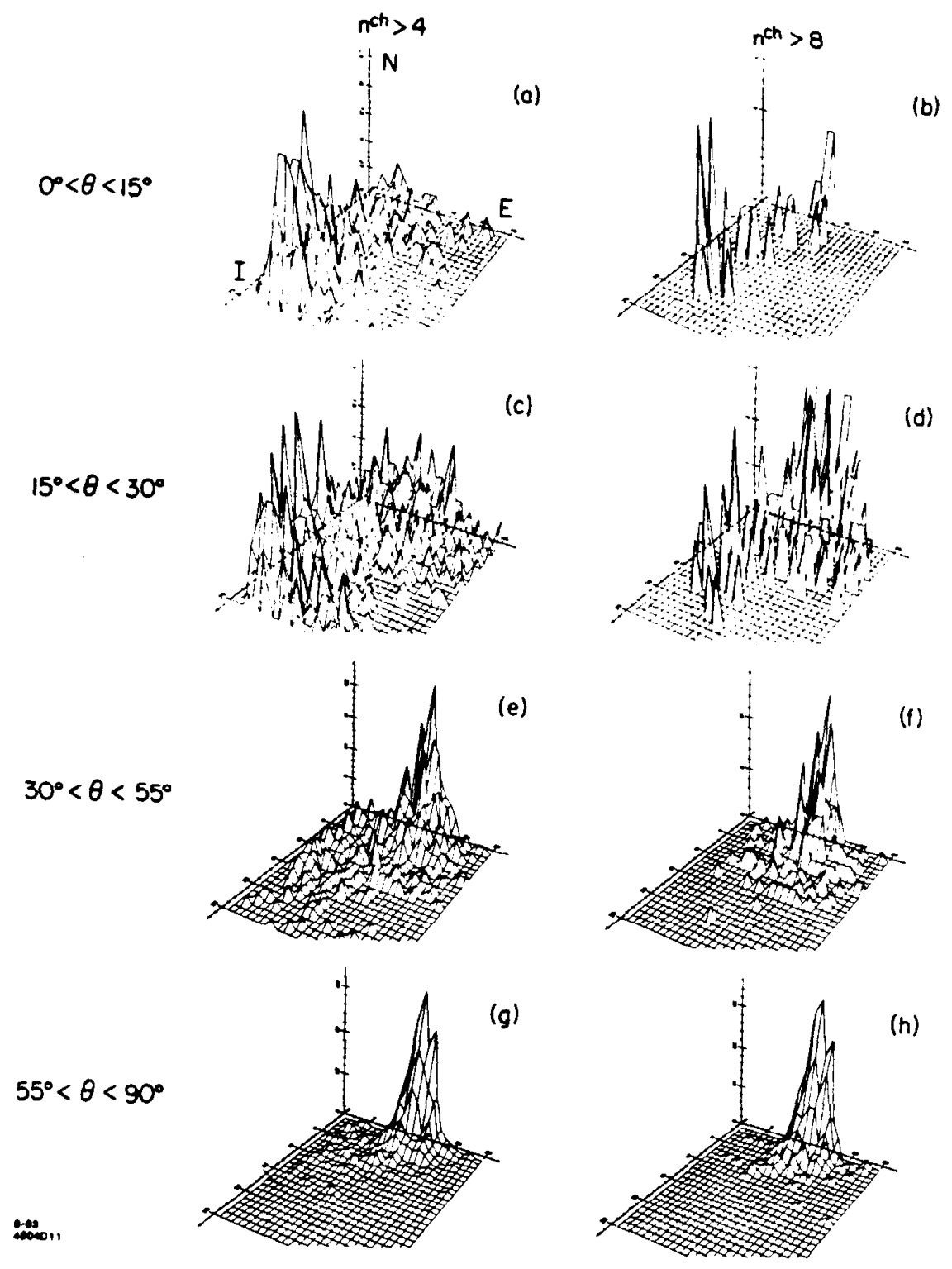

Figure 4-1: 3-dimensional plots of energy versus energy imbalance with $n^{\text {ch }}>4$ and $n^{\text {ch }}>8$ for different angular ranges. Energy bins are $2 \mathrm{GeV}$ and the imbalance bins are 0.04 . Vertical scale is number of events. Annihilation events cluster at higher energies and low imbalances while $2 \gamma$ events cluster at lower energies and higher imbalances. 
for annihilation multi-hadron events. These peaks are reasonably separate from the low energy "shoulders" characteristic of the background processes.

The histograms for low multiplicity events in the low angular ranges, $0^{\circ}-$ $15^{\circ}$ (figs. $\left.a, b\right)$, and $15^{\circ}-30^{\circ}$ (figs. $c$, d) shows no clear peak that can be associated with multi-hadron production. This results from the increases in backgrounds and degraded energy-resolution of the detector at small angles to the beam direction. Therefore an angular cut was required to confine the acceptance to regions with a clearly differentiable annihilation multi-hadron signal.

After cuts based on the above parameters of energy, charged multiplicity, and thrust axis, the remaining sample of events was of sufficient purity that no additional cuts, such as imbalance and vertex position were required. The full details of the procedures and corrections used to evaluate $\mathbf{R}$ follow.

\subsection{Evaluation of $\mathrm{R}$}

Experimental Procedures

From section 3 of Chapter $1 \mathrm{R}$ is measured via the relation

$$
R=\frac{\int_{\theta_{\min }} \frac{d N^{m h}}{d \Omega} d \Omega}{\int_{\theta_{\min }} \frac{d \sigma_{0}^{\mu \mu}}{d \Omega} d \Omega \int \mathcal{L} d t(1+\delta) \epsilon}
$$

where the $\int_{\theta_{\min }} \frac{d N^{m h}}{d \Omega} d \Omega$ is the number of multi-hadron events from $e^{+} e^{-}$annihilation whose thrust axis is greater than $\theta_{\min }, \int_{\theta_{\min }} \frac{d \sigma_{0}^{\mu \mu}}{d \Omega} d \Omega$ is the $\mu$-pair differential cross section integrated over the same angular region, $\int \mathcal{L} d t$ is the integrated luminosity, $\delta$ is the radiative correction, and $\epsilon$ is the acceptance of multi-hadron events to the procedures as determined by Monte Carlo techniques (the Kleiss Monte Carlo was used to generate the quark states and the Lund 
model was used fragment the quarks, for further details see Appendix B).

In order to obtain a high purity sample of annihilation multi-hadron events three cuts have been made to reduce all backgrounds to a low level. The cuts are as follows:

a) Thrust axis as determined from the charged tracks $55^{\circ}<\theta_{p_{\text {thrut }}}<90^{\circ}$;

b) Track multiplicity $>4$;

c) Total event energy $>16 \mathrm{GeV}$.

Approximately half the produced events lie within the fiducial region. The event sample passing the cuts still contains events from background processes. Backgrounds from the $2 \gamma$ process and $\tau$-pair production are estimated using Monte Carlo techniques. The Vermasseren Monte Carlo was used to generate two-photon events and the Kleiss program was used to generate $\tau$-pairs. For further details on the Monte Carlos used see Appendix B. Backgrounds from beam-gas interactions, cosmic rays, etc. are estimated by a visual scan of a sample of the data.

To compute the acceptance of the experimental procedures the Monte Carlo events were put through the same analysis as the data events including a simulation of the trigger system. The acceptance was corrected for differences in the data and Monte Carlo distributions. The sensitivity of the acceptance to Monte Carlo parameters was checked by independently varying several parameters in the Monte Carlo.

A detailed explanation of each of the cuts and the backgrounds and their associated errors follows. 


\section{Thrust Angle Cut}

Two methods are available from the MAC detector data to measure the thrust axis of an event.

i) The thrust axis can be determined using the energy flow data as observed in the calorimetry. In this case the energy vectors, constructed from the magnitude of a shower and its position are used as the basis on which the thrust axis is calculated.

ii) The thrust axis can be determined using the momenta of the charged tracks observed in the central drift chamber.

In principle the biases in the data produced by the use of both these methods can be evaluated with Monte Carlo modeling. In practice method i) based on calorimetry contains systematic biases from three sources; difficulties in angular determinations based on current division measurements, poorly determined energy vectors in the neighborhood of the rift between the central calorimeter and the endcap, and non-uniform response in the endcaps. These effects are difficult to model.

The MAC calorimeters rely on current division for longitudinal position determination. The current division determinations are susceptible to errors both from calibration procedures and from the electrical cross-talk between channels generated in the external connections and in the electronic pre-amplification circuitry. Cross-talk causes spurious and roughly equal pulses to appear at both ends of a wire. This effect results in a systematic increase in the polar angle of the energy thrust axis and increases the number of events appearing to lie within 
a central fiducial region. The cross-talk varies from channel to channel making Monte Carlo modeling difficult and imprecise.

The calorimetry in MAC is extended to small angles with the use of endcaps. The rift between the central detector and the endcap is part of the detector structural support and is not sensitive to the passage of particles. Hence modeling problems arise from the rift because of the discontinuous response of the detector.

In the endcaps there are twelve chambers each subtending $30^{\circ}$ in azimuth. These chambers have a dead space at the edges of about 1-inch in extent and thus at every $30^{\circ}$ in azimuth there is an associated dead region. At the innermost radius of the endcaps about $\frac{1}{3}$ of the circumference is dead area. Incorporation of these effects into the Monte Carlo is difficult and likely to result in substantial systematic error.

Systematic errors in angular determinations are almost completely eliminated for measurements based on thrust-axes measured with the central drift chamber. Tracks with polar angle down to $25^{\circ}$ from the beam line traverse all ten layers of the inner drift chamber and are recorded with close to uniform efficiencies. Polar angles are measured using the stereoscopic views of the chamber and the angles measured are based on the mechanically determined spacings of the wires which are known to high precision. The errors in the position determination and chamber precision are well-modeled by the Monte Carlo. Also included in the modeling are effects due to track losses at high track densities due to track overlaps. 
Systematic errors result if the angular calibration of the inner drift chamber is in error. Polar angles are measured by reconstruction from the stereo-views provided by the differently oriented wire layers. Systematic errors in the relative mechanical positioning of the stereo layers would result in systematic errors in the determination of polar angles. A bound can be set on the errors in mechanical tolerances of $<\frac{1}{3}$ of the measured overall position resolution of the chamber and this corresponds to a maximum systematic error of the polar angle of $\frac{1}{2} \%$. This possible error is already small and is further reduced by the luminosity determination, also entering into the determination of $R$, containing canceling correlated errors arising from the same source. From the above the maximum possible error in $\mathrm{R}$ arising from the mechanical imprecision in wire spacing is $<\frac{1}{4} \%$.

Below about $25^{\circ}$ systematic track losses occur because tracks do not go through all ten layers, resulting in a track detection efficiency which is a function of polar angle. The inner drift chamber also has finite momentum resolution and does not detect neutral particles. These effects result in imprecision in the determination of thrust axis angle and cause events, that if perfectly measured would lie outside the polar angle cut, to be included in the event sample and vice-versa. Given the relatively slowly varying angular distribution of multi-hadron events the gains and losses of events are expected to be nearly canceling. A comparison of Monte Carlo analyses, with and without detector effects, at the chosen polar angle cut of $55^{\circ}$ show $\approx 3 \%$ more events lying within the cut due to detector effects. 
Therefore given the small and well-understood systematic errors of the momentum vectors the angular cut imposed on the data used a thrust axis determined using charged tracks in the inner drift chamber. The final value of $R$ was based on a thrust-axis cut of $55^{\circ}$, which also served to minimize the backgrounds from two-photon contamination.

To evaluate the sensitivity of the final results to the angle chosen for the cut, and to check the foregoing, $\mathbf{R}$ was evaluated for angular cuts of $\cos \theta_{p_{\text {thrust }}}$ ranging from 0.4 to 0.8 . Table 4-1 shows the values of $R$ that corresponded to these various cuts. $R$ is remarkably stable to this cut and accordingly a systematic error associated with this cut of $\frac{1 \%}{2} \%$ was assigned.

Table 4-1

Values of $\mathrm{R}$ normalized at $\cos \theta_{\min }=\mathbf{0 . 6 0}$ obtained for various angular cuts (statistical errors).

$\begin{array}{lccccc}\cos \theta_{\min } & 0.4 & 0.5 & 0.6 & 0.7 & 0.8 \\ \text { Data (background } & 1.013 & 1.005 & 1.000 & 0.993 & 1.013 \\ \text { corrected) } & \pm 0.012 & \pm 0.008 & \pm 0.000 & \pm 0.007 & \pm 0.009\end{array}$

\section{Track and Multiplicity Cuts}

A track is identified by the track finding algorithm as a series of hits in successive layers of the drift chamber constrained to lie along a particle trajectory within the measurement precision.

From visual scanning of uncut events a spurious background of events is created by particles in the halo of the beam showering in the beam pipe, by cosmic 
rays showering in the calorimetry with tracks entering the central drift chamber, and by Bhahba scattered electrons showering at small angles in the "staircase region" of the inner drift chamber. These backgrounds result from the inner drift track reconstruction algorithms constructing spurious tracks out of background hits in the chamber. Spuriously reconstructed tracks, or real background tracks unassociated with a beam-beam interaction, are unlikely to originate from a common vertex. Based on an alysis and visual scanning of uncut data the probability of spurious constrained fits decreases strongly with the number of hits included. A spuriously reconstructed track is likely to have large curvature and therefore low assigned momentum.

Therefore to minimize the inclusion of background events an acceptable track was required to reconstruct to a common vertex and to have either $>5$ hits or 5 hits and a fitted momentum $>1 \mathrm{GeV} / \mathrm{c}$. A further requirement was imposed that at least one track in an event should contain seven or more hits. This further reduced the background from Bhabha events showering in the staircase region. This requirement eliminates $<\frac{1}{10} \%$ of the annihilation multi-hadron sample and therefore has a negligible effect on the acceptance.

As discused in the introduction a track multiplicity cut of $>4$ tracks in an event strongly reduces background from both two-photon and tau decay processes. Two-photon processes have a multiplicity distribution peaking at low multiplicity due to their production at low center-of-mass energy and tau-pair decays have a very small probability to decay into more than four charged particles. A multiplicity cut of $>\mathbf{4}$ tracks was therefore required. With a $\mathrm{BR}[\boldsymbol{\tau} \rightarrow$ 3 charged prongs| $\left.\right|^{1} \approx \frac{1}{7}$ a cut of $n^{c h}>4$ eliminates $\approx 98 \%$ of the taus. The 
uncertainty in the $\tau$ branching ratio introduces a systematic uncertainty into the $\mathrm{R}$ measurement of about $\frac{1}{10} \%$.

After application of the above cuts beam-gas interactions, Bhabha events and cosmics showering in the drift chamber, and beam splash in the detector were all largely removed from the event sample.

A comparison of the multiplicity distribution showed small systematic differences between the data and the Monte Carlo. These differences were taken into account in the final evaluation by scaling the Monte Carlo distribution to give the observed multiplicity. The scaling factor was the mean of the truncated multiplicity distribution $\left(n^{c h}>4\right)$ for the data divided by the corresponding mean for the Monte Carlo. The multiplicity distribution for the data is plotted with the unscaled and scaled Monte Carlo distributions and is shown in Figure 4-2. The Monte Carlo predicts $4.5 \%$ of the events have $\leq 4$ tracks and the scaled Monte Carlo distribution predicts $5.4 \%$ of the events have $\leq 4$ tracks.

A check on the agreement with the Monte Carlo is provided by comparing the fraction of 4 prong events with two particles in both of the opposing hemispheres relative to the direction of the thrust axis. In $2 \gamma$ multi-hadron events the jets are usually both peaked toward the beam line in the same hemisphere. In $\tau$-pair cvents the $\tau$ cannot decay into two charged particles. The fiducial cut reduces the probability that one of the particles in the reaction $\tau \rightarrow$ three-charged particles will be outside the drift chamber acceptance. Thus the 2-2 configuration provides a nearly background-free topology to compare the data and the Monte Carlo modeling. This was done by scanning a sample of data and Monte Carlo events. 

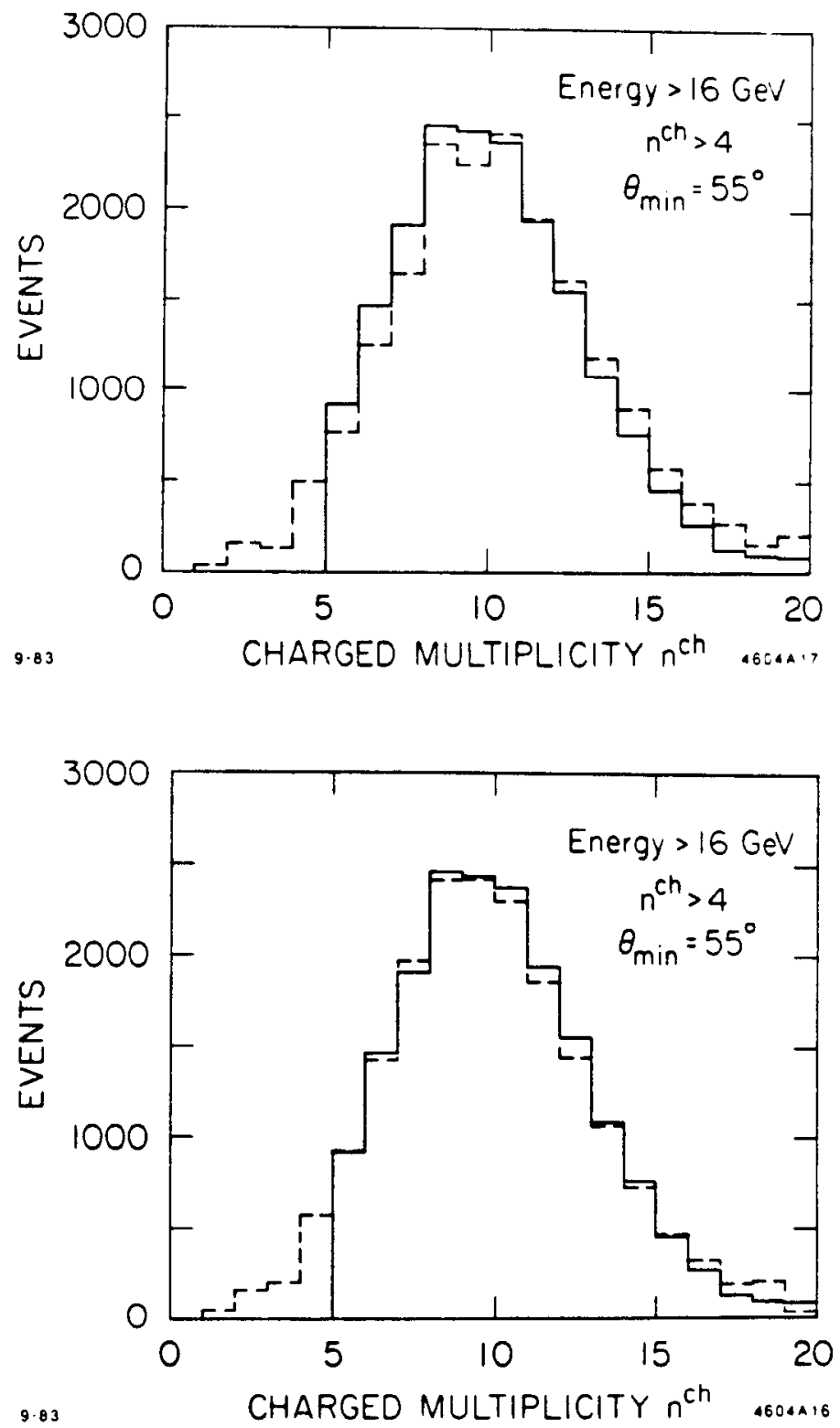

Figure 4-2: Charged multiplicity distributions for data (solid) and Monte Carlo (dashed). In the top plot the Monte Carlo distribution is unscaled and in the bottom plot the Monte Carlo points have been scaled to bring them into agreement with the data. The scaled Monte Carlo distribution is clearly in good agreement with the data. 
The fraction of these events (compared to the fiducially cut sample) was found to be $0.4 \% \pm 0.1 \%$ for the data and $0.3 \% \pm 0.1 \%$ for the Monte Carlo. The data events have a similar energy distribution as the higher multiplicity events indicating they are indeed from the annihilation process and are not from a $2 \gamma$ process.

\section{Energy Cut}

Events passing the multiplicity cut and solid angle cut are further cut on the basis of total calorimetric energy. The reason for this procedure is that even after the angle and multiplicity cuts there is a low energy peak in the energy distribution as shown in Figure 4-3. This double-peaked spectrum is to be expected from a combination of $2 \gamma$ and annihilation events. The $2 \gamma$ events are expected to have low energy and the annihilation events are expected to have nearly twice the beam energy. The total energy cut chosen is $E>16 \mathrm{GeV}$ which is in the valley between the $2 \gamma$ peak and the annihilation multi-hadron peak. The $2 \gamma$ background for $\mathrm{E}>16 \mathrm{GeV}$ is evaluated in a later section and is a small correction. Monte Carlo evaluation of the correction for events lost by the energy cut shows a loss of $1.8 \%$.

The Monte Carlo modeling of the low energy tail was checked with high

multiplicity $\left(n^{\text {ch }}>8\right)$ events. The fraction of events passing the angle cut with $>8$ tracks and $\mathrm{E}<16 \mathrm{GeV}$ is $1.4 \%$ for the background corrected data and $1.0 \%$ for the Monte Carlo. A visual scan of the high multiplicity low energy events shows no detector malfunction in these events. The additional events in the data likely result from higher order QED processes not included in the Monte Carlo but 


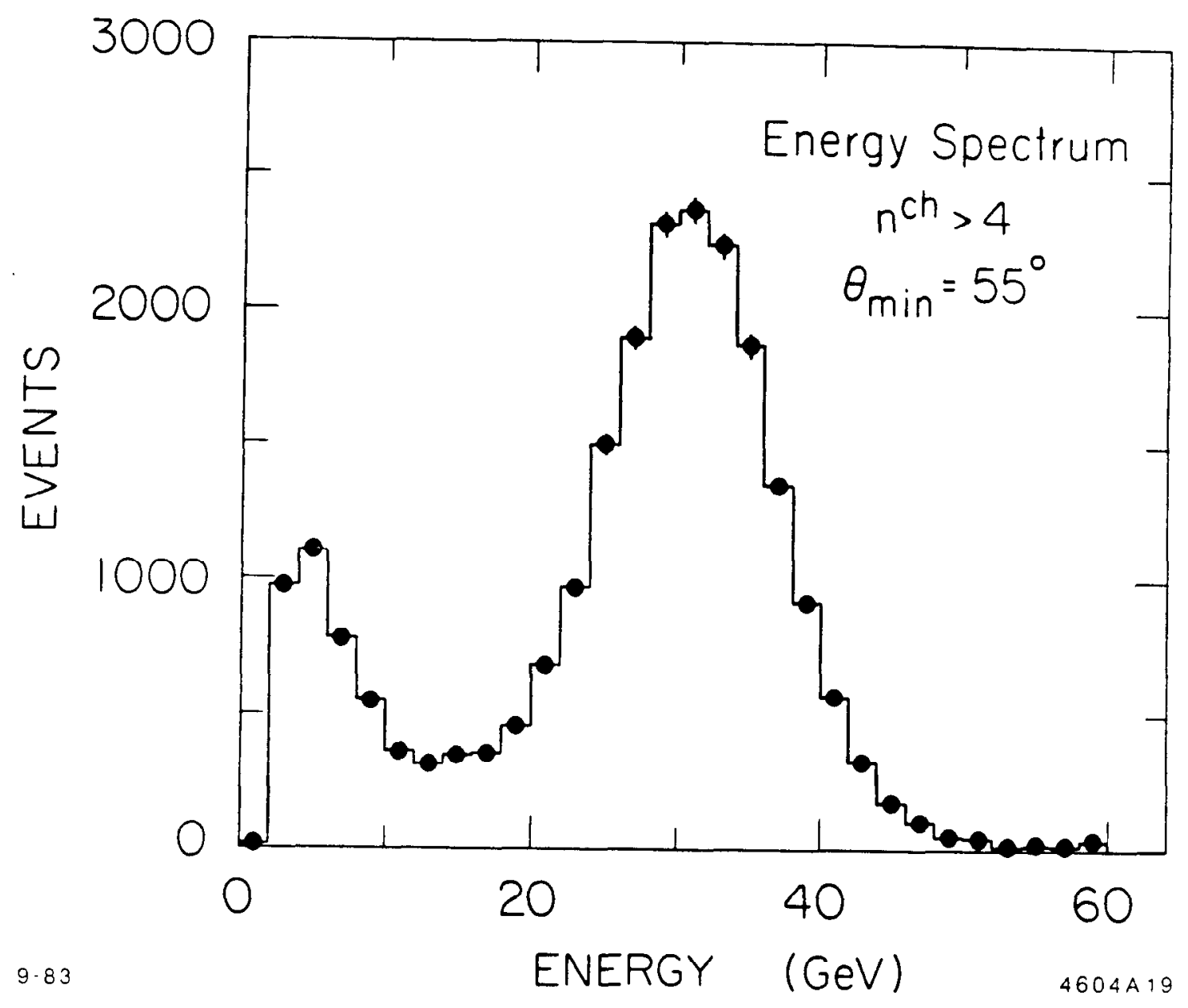

Figure 4-3: $\quad$ Encrgy spectrum for events with $n^{\text {ch }}>4$ and $55^{\circ}<\theta_{p_{t h r u s t}}<$ $80^{\circ}$. 

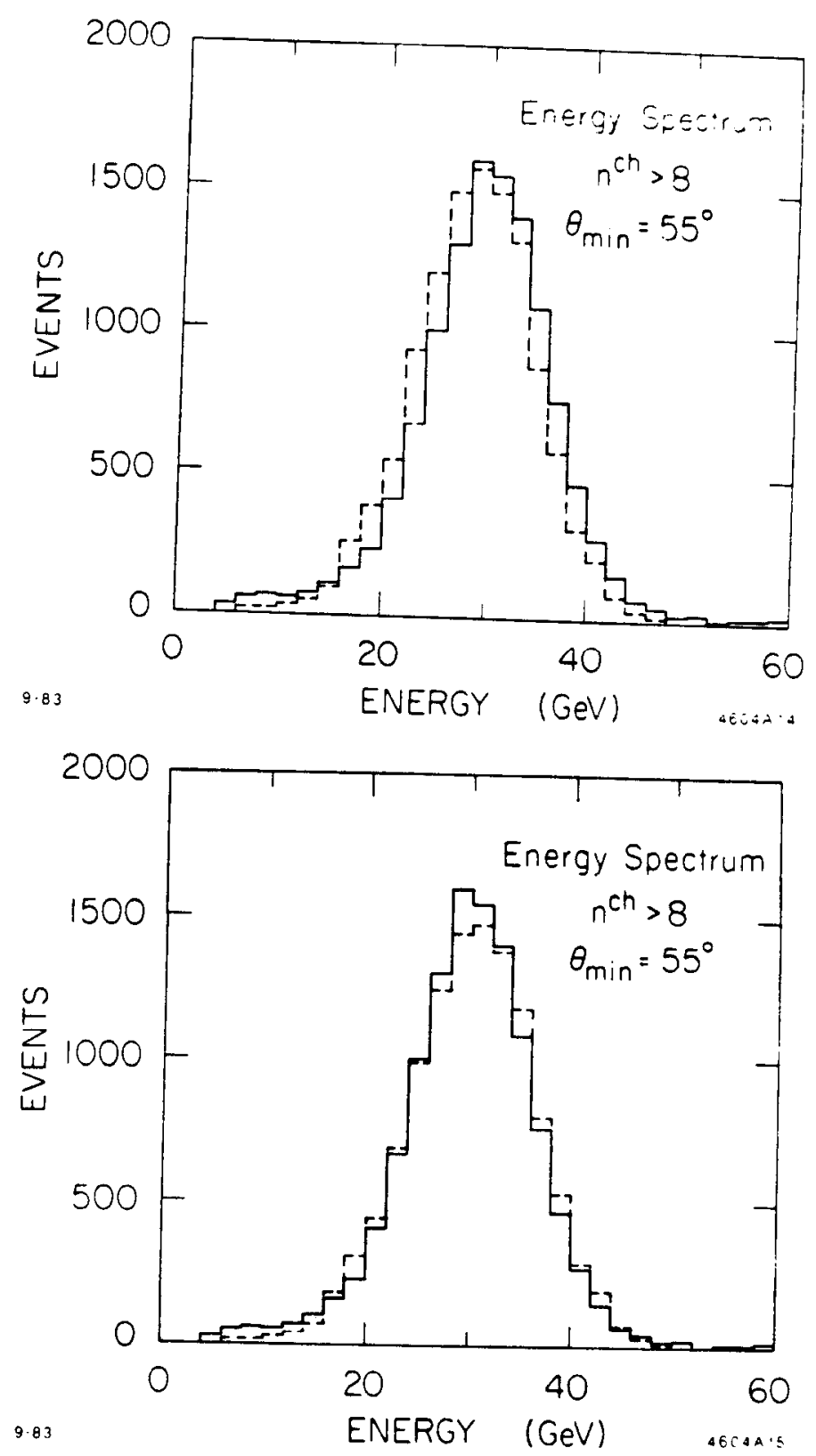

Figure 4-4: Energy spectra for events with $>8$ tracks for data (solid) and Monte Carlo (dashed). In the top plot the Monte Carlo is unscaled and in the bottom plot the Monte Carlo is scaled to bring it into agreement with the data. 
which are included in the calculation of radiative corrections (for further details see Appendix A). The Monte Carlo was therefore assumed to give the correct spectrum for the events it was designed to model. The differences between the spectra were taken into account by scaling the Monte Carlo distribution to bring it into agreement with the data. The scale factor was the mean of the truncated energy distribution (E $>16 \mathrm{GeV}$ ) for events with $>4$ tracks for the background corrected data divided by the corresponding mean for the Monte Carlo. The same scale factor was obtained using events with $>8$ charged tracks. The energy spectra for the data for $>8$ tracks is plotted with the scaled and unscaled Monte Carlo distribution in Fig 4-4. On the basis of the above a systematic error of $1 \%$ is assigned to the energy cut.

The stability of $R$ with respect to the energy cut is shown in Table 4-2.

\section{Table 4-2}

Values of $\mathbf{R}$ for various energy cuts normalized to the nominal value (errors are statistical).

$\begin{array}{lccccc}E_{\text {cut }}(\mathrm{GeV}) & 12 & 14 & 16 & 18 & 20 \\ \text { Data } & 1.002 & 1.000 & 1.000 & 1.002 & 1.005 \\ \text { (background corrected) } & \pm .002 & \pm .002 & \pm .000 & \pm .002 & \pm .003\end{array}$

Backgrounds and Associated Errors

i) General Backgrounds 
In addition to the beam-beam associated multi-hadron backgrounds discussed in the previous sections there are other processes, not included in the Monte Carlo programs, which must be measured experimentally. These background processes are minimized by the choice of cuts chosen in the preceding sections. The estimation of the magnitude and systematic errors of these backgrounds follows.

A sample of about $10 \%$ of the events passing the angular and multiplicity cuts were scanned to find the residual backgrounds from processes such as cosmic rays showering in the calorimeters, Bhabha events showering in the material of the central drift chamber, beam splash (radiation or particles from upstream which spray into the detector), and occasional "blotch events" due to electrical breakdowns in the detector. Less than $4 \%$ of the events with energy less than $16 \mathrm{GeV}$ and less than $\frac{1}{10} \%$ of the events with energy greater than $16 \mathrm{GeV}$ were due to these effects.

ii) Beam-Gas Backgrounds

The correction for beam gas interactions was derived from the distribution of primary vertices along the beam axis (z-coordinate). Events from beam-beam interactions are contained within $\pm 5 \mathrm{~cm}$ of the nominal interaction point. Less than $\frac{3}{10} \%$ of the multi-hadron signal have a vertex outside this range. Events with $|z|>5 \mathrm{~cm}$ are categorized as beam-gas interactions. Since the beam-gas events should be uniformly distributed in $z$, the fraction of beam-gas events with $|z|<$ $5 \mathrm{~cm}$ is easily found. Fig 4-5 shows the distribution of longitudinal position of the primary vertex for events passing the angle cut and the multiplicity cut. From 


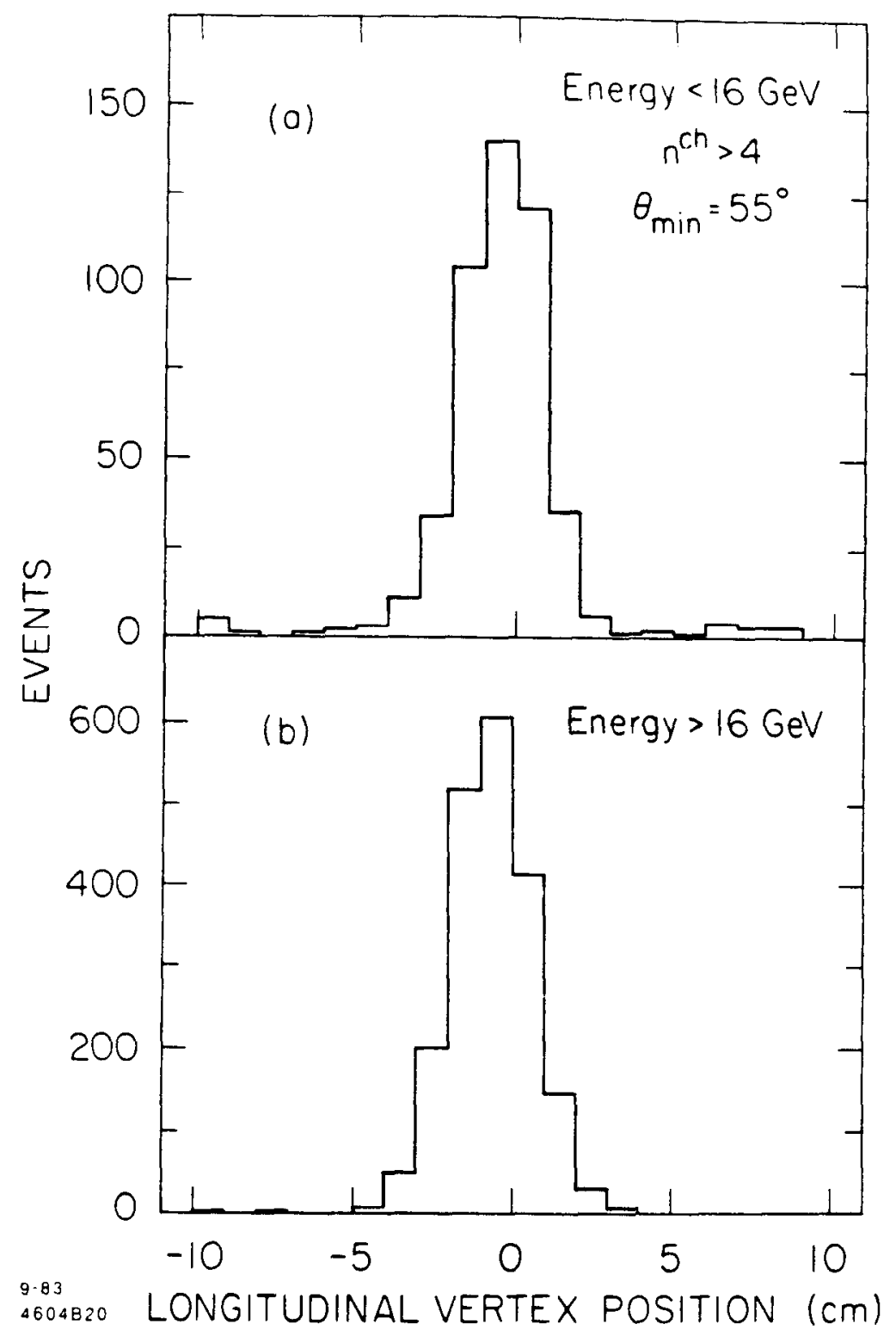

Figure 4-5: Longitudinal position of the primary rertex for events with passing the angle and multiplicity cut. The upper plot is for events with $\mathrm{E}<16 \mathrm{GeV}$ and the bottom plot is for events with $\mathrm{E}>16 \mathrm{GeV}$. 
this data the fraction of beam-gas events in the $\mathrm{E}<16 \mathrm{GeV}$ sample is evaluated to be $\sim 12 \%$ and for the $\mathrm{E}>16 \mathrm{GeV}$ sample the fraction of beam-gas events is $<\frac{1}{10 \%}$.

iii) $2 \gamma$ Background

The number of events observed in the range $\mathrm{E}<16 \mathrm{GeV}$ was compared with a Monte Carlo consisting of hard-scattering and VDM two photon processes. The two photon Monte Carlo is roughly consistent with the data predicting about $30 \%$ fewer events than is actually observed in the energy range $8-16 \mathrm{GeV}$ where trigger efficiencies are more similar to the higher energy events. World data differs by this order of magnitude and this level of precision is not surprising. ${ }^{2-4}$

Table 4-3 calculates the two photon contribution scaled by 1.3 of the two photon Monte Carlo in the range $\mathrm{E}>8 \mathrm{GeV}$. There are obvious uncertainties in this estimate of additional events not included in our two photon Monte Carlos. A conservative error of $100 \%$ is taken for the two photon contribution. With this normalization factor the correction due to subtracting the two photon background is $\sim \frac{1}{2} \%$ with an uncertainty of $\sim \pm \frac{1}{2} \%$. 


\section{Table 4-3}

Contributions to the cross section (in pb) for event categories determined from a best fit to the data.

$\begin{array}{lcc} & 8 \mathrm{GeV}<\mathrm{E}<16 \mathrm{GeV} \mathrm{E}>16 \mathrm{GeV} \\ \text { Two photon (hard scattering) } & 0.9 & 1.1 \\ \text { Two photon (VDM) } & 0.4 & \sim 0 \\ \pi \text { M.C. } & 0.2 & 1.0 \\ \text { Beam-gas } & 0.5 & 0.1 \\ \text { Bhabhas, cosmics, etc. } & 1.3 & 0.2\end{array}$

Normalized annihilation multi-hadron M.C.

Sensitivity of Acceptance to variation of Monte Carlo Parameters

The effects on calculated acceptance due to variations of Monte Carlo parameters are expected to be small. Nevertheless in order to estimate possible systematics we investigated our sensitivity to parameter variations over a reasonable range of values. Due to the large amount of computer time needed to create the data base necessary to investigate several parameters at different values, Monte Carlo events were run through a less comprehensive but much faster detector simulation Monte Carlo. This Monte Carlo was adjusted so that the energy spectrum, multiplicity distribution, and solid angle acceptance were in 
good agreement with the data and full detector Monte Carlo. The parameters varied were thrust cut-off, $\alpha_{s}, p_{\perp}$ relative to the original quark direction, the pseudoscalar to vector meson ratio, the fragmentation function of the heavy quarks, and the fragmentation model. These parameters were chosen because they could potentially affect the acceptance. The thrust cut-off is related to the minimum energy that a gluon may have. It is necessary to model the gluons because gluons fragment into hadrons and affect quantities such as the thrust axis, visible energy, and multiplicity. $\alpha_{s}$ is directly related to the probability that a gluon will be produced thus affecting the charged multiplicity distribution. The momentum of the particles perpendicular to the thrust axis is related to the "jettiness" of an event, the smaller the average $p_{\perp}$ the jettier the event. A highly collimated two-jet event will nearly be totally contained in the detector whereas a more spherical event may have particles outside the detector acceptance and could introduce a systematic error into the thrust-axis measurement. The pseudoscalar to vector meson ratio is important because vector particles are more likely to decay into charged particles which again affects the charged multiplicity distribution.

The breakdown of the errors resulting from variation in the Monte Carlo parameters is shown in Table 4-4. The acceptance is fairly insensitive (about $1 \%$ uncertainty is added to the acceptance error) to reasonable variation in all these parameters and is explained in detail in Appendix B. 
Table 4-4

Change in acceptance due to variation in Monte Carlo parameters.

$\begin{array}{lcc}\text { Quantity } & \text { Range } & \text { \% Change over range } \\ \mathrm{P} /(\mathrm{P}+\mathrm{V}) & 0.35-0.65 & \pm 0.4 \\ \alpha_{s} & 0.12-0.22 & \pm 0.5 \\ T_{\max } & 0.93-0.97 & \pm 0.1 \\ \sigma_{p_{\perp}} & 0.15-0.45 \mathrm{GeV} & \pm 0.4 \\ \text { Fragmentation } & & \\ \text { function } & c f . \text { App. B } & \pm 0.8\end{array}$

\section{Trigger Efficiency}

The Monte Carlo events were put through a hardware trigger simulation program and the efficiency for the selected events was found to be $99.0 \pm 0.1 \%$.

In order to reduce computer time, only events tagged by the offline software as having a momentum thrust axis, $30^{\circ}<0_{p_{\text {thruat }}}<90^{\circ}$ using all the central drift tracks are selected as potential candidates. A spot check is made of events with $\theta_{p_{\text {thrust }}}<30^{\circ}$ as tagged by the offline software to make sure that a large number of events are not missed. This effect is only $\approx \frac{2}{10} \%$ for $n^{c h}>4$ and $\mathrm{E}>16 \mathrm{GeV}$ and is included in the trigger efficiency.

The final value of the trigger efficiency is $.997 \pm .002$ which includes hardware and software filter efficiencies. 


\subsection{DETERMination of $R$}

The number of events passing the cuts is $N^{m h}=18261$ in an integrated luminosity of $80.9 p b^{-1}$. From Table 4-3 the amount of background contamination is $1.1 \%$. Thus the number of annihilation multi-hadron events is:

$$
\int_{\theta_{\min }} \frac{d N^{m h}}{d \Omega} d \Omega=18001 \pm 134 \pm 89
$$

\section{Corrected Acceptance}

In order to find the overall corrections to the acceptance from the cuts used the Monte Carlo events were put through the same analysis as the data. The corrected acceptance is written:

$$
\begin{aligned}
\epsilon & =f_{\theta_{\min }} \cdot f_{n^{c h}} \cdot f_{E_{\text {cut }}} \cdot f_{\text {trig }} \\
& =.836 \cdot 0.991 \cdot 1.003 \cdot 0.987 \\
& =.828
\end{aligned}
$$

where $f_{\theta_{\min }}$ is the fraction of Monte Carlo events within the angular acceptance that pass the multiplicity and energy cut, $f_{n^{\text {ch }}}$ is the adjustment made to the Monte Carlo due to the differences between the data and the Monte Carlo in the multiplicity distribution and $\int_{E_{\text {cut }}}$ is the adjustment made to the Monte Carlo due to the differences between the data and Monte Carlo in the energy distribution. $f_{\text {trig }}$ is the hardware and software trigger efficiency. The major uncertainties in the acceptance arise from the sensitivity due to the multiplicity cut and the energy cut each of which accounts for about $\pm 1 \%$ uncertainty. The numbers and uncertainties entering into $\epsilon$, iucluding sensitivity to Monte Carlo parameters, are shown in Table 4-5. 
Table 4-5

Systematic errors in determining the acceptance.

Quantity

Angular dependence

Multiplicity cut

Energy cut

Trigger efficiency

Sensitivity to $\mathrm{MC}$ parameters

Total systematic error

(summed in quadrature)
\% Error

0.5

1.0

1.0

0.2

1.1

1.9

\section{Luminosity Measurement}

The luminosity was measured using Bhabha scattering at large angles to the beam line. The events were chosen by requiring

i) exactly two central drift tracks;

ii) $>5.7 \mathrm{GeV}$ in the shower chamber;

iii) $<10^{\circ}$ acollinearity angle;

iv) each track $>55^{\circ}$ from the beam line as measured by the central drift.

Additionally a cut is made on the azimuthal angle to eliminate events near the edges where the sextants meet. A sample of the events were visually scanned to 
determine the level of background contamination which is found to be $1.2 \pm 0.8 \%$. The trigger efficiency is very close to 1 and no correction is made. The number of events in the sample is 40426 corresponding to an integrated luminosity of $80.9 p b^{-1}$.

The values and the error assignments used to determine $R$ are shown in Table 4-6.

Table 4-6

Final values and error assignments used in the determination of $R$.

Factor Value Stat. Error (\%) Sys. Error (\%) Source of error

$\int_{\theta_{\min }} \frac{d N^{m h}}{d \Omega} d \Omega \quad 18001 \quad 0.7 \quad 0.5 \quad 2 \gamma$ events
$\int \mathcal{L} d t\left(p b^{-1}\right)$
80.9
0.5
1.6
trigger eff.

and rad. corr.

$1+\delta$

1.422

0.8

rad. corr.

$\epsilon$

0.828

0.4

1.9

cf. Table 4-5

$\int_{\theta_{\min }} \frac{d \sigma_{0}^{\mu \mu}}{d \Omega} d \Omega(p b) \quad 49.3$

Using the formula for $R$ given in section 2 and the values given in Table 4-6 we obtain:

$$
R=3.83 \pm 0.04(\text { stat. }) \pm 0.10(\text { syst. })
$$


This result can be interpreted via the formula

$$
R=3 \sum_{\substack{\text { quark } \\ \text { flavors }}} e_{q}^{2}\left(1+\frac{\alpha_{s}}{\pi}\right) .
$$

as yielding a value for $\alpha_{s}$ of

$$
\alpha_{s}=0.14 \pm 0.09
$$

A value of $\mathrm{R}$ has also been derived from the MAC detector using events distributed over the full MAC angular acceptance. Because of the difficulties associated with the separation of multi-hadron events from background processes at small angles to the beam-line, this analysis required a rather elaborate set of cuts to define the multi-hadron sample. These cuts can be summarized as:

$\mathrm{E}>12 \mathrm{GeV}$,

$E_{\perp}>7.5 \mathrm{GeV}$

Energy imbalance $=\left(\sum_{i}\left|\vec{E}_{i}\right| / \sum_{i} E_{i}\right)<0.65$, $n^{c h}>2$,

Primary vertex position $<5 \mathrm{~cm}$,

Scalar sum of momenta $>2 \mathrm{GeV} / \mathrm{c}$.

Additional cuts were made on the average number of hits per central drift track, and on energy-density in the calorimeters. These cuts were further supplemented by visual scanning of events at the boundaries of the acceptance criteria.

The value of $R$ was determined via the ratio of total cross sections. However the sources of systematic error increase with this procedure. The value of $R$ using 
the full detector acceptance ${ }^{5}$ is $^{\dagger}$

$$
R=3.91 \pm 0.03 \pm 0.15
$$

in excellent agreement with our result.

Determination of an angular distribution for the annihilation process using the inner-drift chamber or charge division measurements is limited in precision by the systematics previously discussed. Our measurement provides a good determination of the events at large angles and the second determination over most of the solid angle is not heavily dependent on charge division determinations. The two MAC R measurements are in excellent agreement and may be combined to provide a check of the angular distribution for multi-hadron production as a function of $\cos \theta$. A cross section measurement (as opposed to an asymmetry measurement) only depends on even powers of $\cos \theta$. Annibilation events via single photon exchange do not contain terms higher than $\cos ^{2} \theta$ and accordingly terms in $\cos ^{4} \theta$ and higher will be small. The cross section can therefore be expressed in the form

$$
\frac{d \sigma^{m h}}{d \Omega} \sim 1+A \cos ^{2} \theta
$$

${ }^{\dagger}$ The quoted value is $R=3.91 \pm 0.03 \pm 0.11$. The systematic errors associated with the luminosity measurements and radiative corrections are almost identical in the two measurements. However systematic errors associated with multiplicity, energy and imbalance cuts, and with the background subtractions of two-photon events substantially increase at small acceptance angles. We estimate the overall systematic errors from these to be 2.5 times greater than the corresponding overall errors for our angular range. The assigned errors however are close to those used for our angular range and therefore to put the two determinations on a comparable basis we have increased the above systematics by a factor of 2.5 . 
Using the two separate MAC measurements to solve for $A$ gives $A=0.92 \pm$ 0.10 , in good agreement with the theoretical value of 1 .

\subsection{COMPARISON WITH OTHER EXPERIMENTS}

Other $R$ measurements ${ }^{6-9}$ at PEP/PETRA energies use similar event selection criteria. A charged multiplicity cut of $>4$ prongs is nearly universal as a cut against $\tau$-pair events. Also, an energy cut $\left(\approx \frac{1}{2} \sqrt{s}\right)$ is used to remove beam gas events and most of the $2 \gamma$ physics. There are additionally fiducial cuts based on parameters such as $E_{\|}, E_{\perp}$, or thrust axis. The events passing the selection criteria are scanned to determine the sample purity. Monte Carlo programs are used to estimate the acceptance of the cuts and to provide a correction to the number of events. Table 4-7 shows a comparison of $R$ measurements from PEP and PETRA experiments radiatively corrected to $O\left(\alpha^{3}\right)$. For comparison purposes the MAC R-value has been increased by $1.7 \%$ to remove the higher order corrections. 
Table 4-7

$$
\begin{aligned}
& \text { R-values at PEP and PETRA } \\
& \text { (radiatively corrected to } \left.O\left(\alpha^{3}\right)\right) \\
& \quad \text { Energy range } R_{ \pm} \Delta R_{\text {stat }} \pm \Delta R_{\text {sys }}
\end{aligned}
$$

TASSO $\quad 14.0-36.7 \quad 4.01 \pm 0.03 \pm 0.20$

$\begin{array}{lcc}\text { JADE } & 14.0-36.7 & 3.97 \pm 0.05 \pm 0.10 \\ \text { MARK J } & 12.0-36.7 & 3.84 \pm 0.05 \pm 0.22 \\ & & \\ \text { MARK II } & 29.0 & 3.90 \pm 0.05 \pm 0.25 \\ & & \\ \text { MAC } & 29.0 & 3.89 \pm 0.04 \pm 0.10\end{array}$

All of the above determinations of $\mathrm{R}$, except for the JADE value, contain a larger quoted systematic error than for our result. This difference arises because the smaller angular coverage of their detectors required cuts to be made close to the edges of their acceptance, thus making their corrections less certain. The value quoted by JADE is to $O\left(\alpha^{3}\right)$ and does not include a correction or systematics for the higher order corrections included in our result. The overall agreement between experiments is excellent and well within quoted errors. 


\section{REFERENCES}

1. Ford, W. T., et al., "Physics with the MAC Detector", SLAC Report No. SLAC-2986, 1982.

2. Bartel, W., et al., DESY Report No. DESY 81/048, 1981.

3. Brandelik, R., et al., Phys. Lett. 107B, 290 (1981).

4. Wagner, W., DESY Report No. DESY 80/102, 1880.

5. Heltsley, B.K., PhD. thesis (unpublished), 1983.

6. Bartel, W., et al., DESY Report No. DESY 83-050, 1983.

7. Brandelik, R., et al., Phys. Lett., 113B, 499 (1980).

8. Heinzelman, G., "Recent Results from JADE at PETRA", in Proceedings of the 21st International Conference on High Energy Physics, Paris, July 26-31, 1982.

9. Trilling, G., "Mark II Results at PEP", in Proceedings of the 21st International Conference on High Energy Physics, Paris, July 26-31, 1982. 


\section{Chapter 5}

\section{Conclusions}

The value of $R$ has been measured via the ratio of differential cross sections integrated over an angular range using the MAC detector. The measured value of $\mathrm{R}$ is

$$
\mathrm{R}=3.83 \pm 0.04(\text { stat } .) \pm 0.10(\text { syst } .)
$$

The result is the most precise to date and is in good agreement with other experiments. The value of $\mathrm{R}$ is about one and a half standard deviations from the value predicted using the simple quark parton model with 5 quarks. The value of $\alpha_{s}$ is found to be $0.14 \pm 0.09$. The angular distribution was checked for a $1+A \cos ^{2} \theta$ form and a value of $A=0.93 \pm 0.10$ was obtained compared with the canonical value of 1 . The small systematic error in the value of $R$ is due to the fact that with a nearly full $4 \pi$ solid angle detector a fiducial cut can be made which avoids lesser-known regions of the physics and the detector while maintaining a reasonable acceptance.

Three roughly coequal sources dominate the systematic error. These errors are from; acceptance including backgrounds, luminosity determination, and radiative QED effects. In order to significantly reduce the total uncertainty of $R$, reduction in all these errors is necessary.

One major source of uncertainty in the acceptance is in the number of multihadron events due to the two photon process. The measured cross section of the $2 \gamma$ process in this experiment is about a factor of 2.5 greater for low energies than the prediction from the hard scattering model. Clearly more work is needed in this area on both the experimental and theoretical fronts. The Monte Carlo 
modelling of the acceptance is also a major source of uncertainty which affects the multi-hadrons and Bhabhas. Improvement in the Monte Carlo will involve detailed adjusting of the Monte Carlo parameters so that the distributions of the data and Monte Carlo are more nearly matched. For an ultra-precise $(\approx 1 \%)$ $\mathbf{R}$ measurement the thrust axis may not be the optimal method for making a fiducial cut. This is because the thrust axis of events with two jets in the same hemisphere (typical of two photon events and highly radiative one photon events) is highly sensitive to changes in the vectors used in the calculation. At the $\approx 2 \%$ level this is not a problem.

Another major systematic uncertainty are the radiative corrections. These effects include errors from the following sources: uncertainty of low energy $R$ measurements of other experiments which affects the nominal energy measurement because hard bremsstrablung lowers the center-of-mass energy significantly, vacuum polarization due to threshold effects, and higher order QED effects obtained from summing leading logs.

Thus far PEP has run at one energy and while this is useful for obtaining high statistics measurements it is not useful for studying energy dependent effects. These effects include weak effects which scale as $8^{2}$ at PEP energies and QCD effects which vary logarithmically with the center of mass energy. In the future, if PEP runs at different energies, it will be useful to study energy dependent quantities of the physics processes and of the detector response. 


\section{Appendix A}

\section{Radiative Corrections}

\section{A.1 General Remarks}

To do a precision measurement of $\mathbf{R}$ requires that higher order (radiative) QED processes be taken accurately into account for both the luminosity measurement (Bhabha scattering) and the annihilation multi-hadron production.

The simplest corrections to the lowest order process are the well-known $O\left(\alpha^{3}\right)$ processes which can be written in a form that multiplies the $O\left(\alpha^{2}\right)$ cross section:

$$
C_{\alpha^{3}}=1+\delta_{\alpha^{3}}=1+\delta_{v e r t}^{e}+\delta_{v e r t}^{f}+\delta_{v a c}+\delta_{\gamma}^{e}+\delta_{\gamma}^{f}
$$

The vertex corrections are given by

$$
\begin{aligned}
& \delta_{v e r t}^{e}=\frac{2 \alpha}{\pi}\left(\frac{3}{4} \ln \frac{\left|q^{2}\right|}{m_{e}^{2}}-1+\frac{\pi^{2}}{6}\right), \\
& \delta_{v e r t}^{f}=\frac{2 \alpha}{\pi}\left(\frac{3}{4} \ln \frac{\left|q^{2}\right|}{m_{f}^{2}}-1+\frac{\pi^{2}}{6}\right),
\end{aligned}
$$

where $m_{e}, m_{f}$ are the electron and final state particle masses (if quarks then in GeV: $m_{u}=m_{d}=0.3, m_{o}=0.5, m_{c}=1.6, m_{b}=5.0$, actually the quark masses are not known but they only appear in logarithms so the answer is only 
slightly sensitive to the quark mass) and $q^{2}$ is the square of the momentum transfer (negative is time-like).

The vacuum polarization can be written as the sum of three contributions, the lepton contribution $\Re I_{l}$, the hadron continuum $\Re \Pi_{\text {cont }}$, and vector meson resonances $\Re \Pi_{\text {res. }}$. Here means the real part, and $\Pi$ is the vacuum polarization amplitude of the photon propagator. The vacuum polarization is given by:

$$
\delta_{v a c}=2\left(\Re \Pi_{l}+\Re \Pi_{\text {cont }}+\Re \Pi_{\text {res }}\right)
$$

with,

$$
\Re \Pi_{l}=\Re \sum_{\text {leptono }}\left[-\frac{\alpha s}{3 \pi} \wp \int_{0}^{\infty} \frac{R_{l}\left(s^{\prime}\right) d s^{\prime}}{s^{\prime}\left(s^{\prime}-s^{2}\right)}-\frac{i \alpha}{3} R_{l}(s)\right]
$$

where $s=4 E_{\text {beam }}, \wp$ is the principle part of the integral, $m_{l}$ is the mass of the lepton (in GeV; $m_{e}=0.000511, m_{\mu}=0.105, m_{\tau}=1.782$ ), For $x=4 m_{l}^{2} / q^{2}<1$ the result is,

$$
\begin{aligned}
\Re \Pi_{l} & =\frac{-\alpha}{3 \pi} \sum_{\text {leptons }} \frac{5}{3}+x+\frac{(1-x)\left(1+\frac{\pi}{2}\right)}{|x-1|^{\frac{1}{2}}} \ln \left(\frac{1-|x-1|^{\frac{1}{2}}}{1+|x-1|^{\frac{1}{2}}}\right) \\
\lim _{|q 2|>4 m_{l}^{2}} \Re \Pi_{l} & =\sum_{\text {leptons }} \frac{\alpha}{\pi}\left(\frac{1}{3} \ln \frac{\left|q^{2}\right|}{m_{l}^{2}}-\frac{5}{8}\right)
\end{aligned}
$$

Also,

$$
\begin{aligned}
\Re \Pi_{\text {cont }} & =\frac{\alpha}{3 \pi} \sum_{i} \Delta R_{i} \ln \left|\frac{q^{2}}{q_{i}^{2}}-1\right| \\
\Re \Pi_{\text {res }} & =\frac{3 q^{2}}{\alpha} \sum_{r e s} \frac{\Gamma_{e e}}{m_{r e s}} \cdot \frac{q^{2}-m_{r e s}^{2}+\Gamma_{r e s}^{2}}{m_{r e s}^{2} \Gamma_{r e s}^{2}+\left(q^{2}-m_{r e s}^{2}\right)^{2}} \\
\lim _{\left|q^{2}\right|>4 m_{r e s}^{2}} \Re \Pi_{\text {res }} & =\frac{3}{\alpha} \sum_{\text {res }} \frac{\Gamma_{e e}}{m_{r e s}} \frac{1}{1-\frac{m_{r e e}^{2}}{q^{2}}}
\end{aligned}
$$


where $\Delta R_{i}$ is the step increase in $\mathrm{R}$ occuring at $\left|q_{i}^{2}\right|$ measured from experimental data, $\Gamma_{e e}$ is the width of the resonance (vector meson) into $e^{+} e^{-}$, and $m_{r e s}$ is the mass of the resonance.

The bremsstrahlung corrections are:

$$
\begin{aligned}
& \delta_{\gamma}^{e}=\frac{2 \alpha}{\pi}\left(\ln \frac{\left|q^{2}\right|}{m_{e}^{2}}-1\right)\left[\ln \frac{k_{\text {max }}^{e}}{E_{\text {beam }}}-\frac{1}{2} \ln \left(1-\frac{k_{\text {max }}^{e}}{E_{\text {beam }}}\right)-\frac{1}{2} \frac{k_{\text {max }}^{e}}{E_{\text {beam }}}\right] \\
& \delta_{\gamma}^{f}=\frac{2 \alpha}{\pi}\left(\ln \frac{\left|q^{2}\right|}{m_{f}^{2}}-1\right)\left[\ln \frac{k_{\text {max }}^{f}}{E_{\text {beam }}}-\frac{k_{\text {max }}^{f}}{E_{\text {beam }}}+\frac{1}{4}\left(\frac{k_{\text {max }}^{f}}{E_{\text {beam }}}\right)^{2}\right]
\end{aligned}
$$

where $E_{\text {beam }}$ is the beam energy, $k_{\max }^{e}$ is the maximum energy photon that can be emitted by the electron, and $k_{\max }^{f}$ is the maximum energy photon that can be emitted by the final state fermion. Note that the initial state bremsstrahlung term, $\delta_{\gamma}^{e}$, takes into account the increase in the cross section due to the lower center-of-mass energy. The following section gives a detailed discussion of the $O\left(\alpha^{3}\right)$ contributions and higher order corrections.

\section{A.2 Detailed ANalysis of $O\left(\alpha^{3}\right)$ and Higher Order Contributions}

The cuts placed on the multi-hadron events and Bhabha events will affect the radiative corrections. In the case of large angle Bhabha scattering the fiducial volume cut, both tracks $>55^{\circ}$ from the beam line, puts a limit on the $q^{2}$ of the reaction, $179(\mathrm{GeV} / \mathrm{c})^{2}<\left|q^{2}\right|<662(\mathrm{GeV} / \mathrm{c})^{2}$ for the exchange process with an average $q^{2}$ of $\left\langle q^{2}\right\rangle=-307(\mathrm{GeV} / \mathrm{c})^{2}$. Since the Bhabha cross section is dominated by the exchange diagram we will assume for calculational purposes that the Bhabha events have an average $q^{2}$ of $-307(\mathrm{GeV} / \mathrm{c})^{2}$. The multi-hadron events have an average $q^{2}$ of $780(\mathrm{GeV} / \mathrm{c})^{2}$ which is less than the beam energy squared because of initial state radiation. 
Uncertainties in the $O\left(\alpha^{3}\right)$ calculation result from uncertainties in the vacuum polarization, lower energy measurements of $\mathbf{R}$, collinearity requirement (applies to Bhabha events only), and possible "new physics".

\section{Vacuum Polarization}

The contributions to the vacuum polarization from vector meson resonances were calculated using the values in Table A-1. The values in the table refer to annihilation multi-hadron events, Bhabha events give similar results. The uncertainty in the decay width contributes to the uncertainty of the vacuum polarization.

Table A-1

Contributions to vacuum polarization from vector meson resonances.

$\begin{array}{cccc}\text { Resonance } & m_{\text {res }}(\mathrm{GeV}) & \Gamma_{e e}(\mathrm{keV}) & \Re \Pi_{\text {res }} \\ \rho & 0.77 & 6.5 \pm 0.7 & .0035 \pm .0004 \\ \omega & 0.78 & 0.8 \pm 0.05 & .0004 \pm .0000 \\ \phi & 1.02 & 1.3 \pm 0.07 .0005 \pm .0000 \\ \psi & 3.10 & 4.8 \pm 0.5 & .0006 \pm .0001 \\ \psi^{\prime} & 3.68 & 2.1 \pm 0.2 & .0002 \pm .0000 \\ \Upsilon & 0.46 & 2.0 \pm 0.3 & .0001 \pm .0000 \\ \Upsilon^{\prime} & 10.02 & 0.5 \pm 0.2 & .0000 \pm .0000 \\ \text { All Resonances } & - & - & .0054 \pm .0004\end{array}$


Table $A-2$ shows the steps used in the continuum contribution to the vacuum polarization. The considerable systematic error of the low energy $\mathbf{R}$ measurements propagates through the vacuum polarization calculation. Again the table refers to annihilation multi-hadron production.

Table A-2

Contribution to vacuum polarization from the hadron continuum.

$$
\begin{array}{ccc}
q_{i}^{2}(\mathrm{GeV})^{2} & \Delta \mathrm{R} & \Re \Pi_{\text {cont }} \\
0.08 & 0.7 \pm 0.3 & 0.0062 \pm 0.0025 \\
1.5 & 1.3 \pm 0.5 & 0.0063 \pm 0.0021 \\
13.7 & 2.0 \pm 0.5 & 0.0062 \pm 0.0012 \\
64 & -0.4 \pm 0.5 & -0.0008 \pm 0.0005 \\
121 & 0.3 \pm 0.2 & 0.0004 \pm 0.0002 \\
\text { All Steps } & - & 0.0183 \pm 0.0035
\end{array}
$$

The possibility of "new physics" will also add to the vacuum polarization. In the worst case scenario (from our perspective) a new heavy lepton and the top quark both with mass $\approx 20 \mathrm{GeV}$ will contribute to the vacuum polarization. At PEP energies new physics has $x=4 \mathrm{~m}^{2} / q^{2}>1$ so the following form for $\Pi$ must be used,

$$
\Re \Pi_{n e w}=\frac{\alpha}{3 \pi}\left[\sqrt{|x-1|}(2+x) \tan ^{-1}\left(\frac{1}{\sqrt{|x-1|}}\right)-x-\frac{5}{3} \mid .\right.
$$

The same expression is used to calculate the contribution from a heavy lepton 
and the $t$ quark (with appropriate charge and color factors taken into account) and ieglect the latters vector meson resonance contribution. The value of $\Re \Pi_{n e w}$ is $-0.03 \%$ for multi-hadron production.

The value for the vacuum polarization is calculated by summing the individual contributions. The error in the vacuum polarization is calculated by adding the various contributions in quadrature. Thus, $\delta_{v a c}^{B h a b h a}=0.090 \pm 0.004$ and $\delta_{v a c}^{m . h .}=0.098 \pm 0.004$.

$\underline{\text { Higher Orders }}$

To calculate higher order processes is quite formidable, in fact a consistent calculation to $O\left(\alpha^{4}\right)$ for the annihilation process has never been done. Therefore techniques which can at least give some of the higher order terms ${ }^{1-2}$ are used. To justify the form of the correction some intuitive motivation will be given.

One of the techniques used to model higher order processes is called exponentiation. This approximation technique models multiple photon emission and is written:

$$
C_{e x p}=\left(1+\delta_{v e r t}^{e}+\delta_{v e r t}^{f}+\delta_{v a c}\right) e^{\delta_{\gamma}^{e}+\delta_{\gamma}^{f}}
$$

However, exponentiation gives a systematically low correction when compared to more comprehensive methods as will now be described.

To get the higher orders of the vacuum polarization all the bubble insertions of the photon propagator are summed to yield

$$
\sum_{n=0}^{\infty} \Pi^{n}=\frac{1}{1-\Pi}
$$


Since the photon propagator enters in as the square in the cross section the correction to the lowest order cross section becomes:

$$
C_{\text {improved }}=\frac{1}{|1-\Pi|^{2}} e^{\delta_{r}}
$$

with $\delta_{r}=\delta_{v e r t}^{e}+\delta_{v e r t}^{f}+\delta_{\gamma}^{e}+\delta_{\gamma}^{f}$. In fact one can do even better than this by summing all leading log terms to obtain a form that is consistent to $O\left(\alpha \ln q^{2}\right)^{n}$ and has no terms of $\ln q^{2}$ with a power greater than that of $\alpha$. The final form used is written:

$$
C_{\text {leadins }}=\frac{1}{|1-\Pi|^{2}}(1-\Re \Pi)^{-\delta_{r} / \Re \Pi} .
$$

By expanding $C_{\text {leading }}$ the lowest order correction $C_{\alpha^{3}}$ is trivially recovered. Table A-3 shows the contributions to $\delta_{\alpha^{8}}$. The entries for Bhabba scatttering assume a bremsstrahlung energy cut-off of $0.2 E_{\text {beam }}$. The entries for multi-hadron production assume a bremsstrahlung energy cut-off of $0.7 E_{\text {beam }}$.

\section{Table A-3}

\begin{tabular}{|c|c|c|c|c|c|c|c|c|}
\hline Process & $\begin{array}{c}\left\langle q^{2}\right\rangle \\
(\mathrm{GeV} / \mathrm{c})^{2}\end{array}$ & $\delta_{v a c}$ & $\delta_{v e r t}^{e}$ & $\delta_{v e r t}^{f}$ & $\delta_{\gamma}^{e}$ & $\delta_{\gamma}^{f}$ & $C_{\alpha^{3}}$ & $C_{\text {lesding }}$ \\
\hline $\begin{array}{l}\text { Bhabha } \\
\text { scattering }\end{array}$ & -307 & .080 & .076 & .076 & -.148 & -.069 & 1.025 & 1.025 \\
\hline $\begin{array}{l}\text { multi-hadron } \\
\text { production }\end{array}$ & 780 & .009 & .078 & .051 & -.010 & -.045 & 1.174 & 1.185 \\
\hline
\end{tabular}

Radiative corrections in Bhabha scattering and multi-hadron production 
Uncertainties in the Leading Log Calculation

The $O\left(\alpha^{3}\right)$ radiative correction correctly takes into account the change in the cross section due to initial state radiation:

$$
\sigma \sim \frac{1}{s\left(1-k_{\gamma}^{e} / E_{\text {beam }}\right)}=\frac{1}{s^{\prime}} .
$$

The change in the cross section due to the photon emission is given by

$$
\begin{aligned}
\delta_{\text {extra }} & =\delta_{\gamma}^{e}\left(\sigma \propto \frac{1}{s}\right)-\delta_{\gamma}^{e}(\sigma \propto \text { constant }) \\
& =\delta_{\gamma}^{e}-\delta_{\gamma}^{f}
\end{aligned}
$$

where for now $\delta_{\gamma}^{f}$ is evaluated at $k_{\max }^{e}$ and not $k_{\max }^{f}$. The leading $\log$ form contains a factor $\approx e^{\delta_{\gamma}^{e}}$ which has terms beyond the $1 / s^{\prime}$ kinematic enhancement. Thus the leading log approximation increases the kinematic enhancement by

$$
f_{k . e .} \approx\left(e^{\delta_{\gamma}^{e}}-e^{\delta_{\gamma}^{f}}\right)-\left(\delta_{\gamma}^{e}-e_{\gamma}^{f}\right)
$$

For hard bremsstrahlung $f_{k . e .}$ is large and significantly amplifies the cross section by more than $\frac{1}{8}$. The physics motivation for this term is not clear and we can use $f_{k . e .}$ as a measure of the uncertainty of the treatment of hard photons by the leading $\log$ method. For Bhabha events with $\theta>55^{\circ}$ and $<10^{\circ}$ acollinearity the correction is $\approx 0.3 \%$. In the case of annihilation multi-hadron production $\left(k_{\text {max }}^{e}=0.7 E_{\text {beam }}\right)$ the effect of "kinematic enhancement" is $\approx 0.4 \%$.

The treatment used to calculate radiative corrections does not include terms where the power of $\alpha$ is larger than the power of $\ln \frac{q^{2}}{m_{e}^{2}}$. The leading term of this type has the form $\sim \alpha^{2} \ln \frac{q^{2}}{m_{e}^{2}}$. An estimate of the uncertainty in the leading log calculation can be obtained by looking at

$$
C_{h . o .}=C_{\text {leading }}-C_{\alpha^{3}} \approx \frac{1}{2} \delta_{r}^{2}+\frac{5}{4} \delta_{v a c}+\frac{3}{4} \delta_{v a c}^{2}
$$


where $C_{h . o}$. represents the higher order $\left(>O\left(\alpha^{3}\right)\right)$ terms. The uncertainty in $C_{h . o .}$ can be written as

$$
\Delta C_{h . o .}=\sum_{\delta_{i}} \Delta C_{\delta_{i}}=\sum_{\delta_{i}} \frac{\partial C_{h . o .}}{\partial \delta_{i}} \frac{\delta_{i}}{\ln \frac{q^{2}}{m_{i}^{2}}}
$$

where $\delta_{i}=\delta_{v e r t}^{e, f}, \delta_{\gamma}^{e, f}, \delta_{v a c}$ and we have assumed that the uncertainty in $\delta_{i}$ is $\sigma_{\delta_{i}} \approx \delta_{i} / \ln \frac{q^{2}}{m_{e}^{2}}$ which is $O\left(\alpha^{2} \ln \frac{q^{2}}{m_{i}^{2}}\right)$. Table A-4 shows the contributions to $\Delta C_{h . o .}$. To obtain the higher order uncertainty we first sum the final state uncertainties $\left(\Delta C_{\delta_{v e r t}^{f}}\right.$ and $\left.\Delta C_{\delta_{\gamma}^{f}}\right)$ because they are correlated before performing the quadrature sum with the other terms. The uncertainty in the higher order terms is thus estimated to be $0.04 \%$ for Bhabhas and $0.16 \%$ for multi-hadrons.

Table A-4

Uncertainties in the leading log approximation for multi-hadron production and Bhabha scattering

$\begin{array}{ccc}\delta & \Delta C_{\delta_{i}} \text { M.H. } & \Delta C_{\delta_{i}} \text { Bhabha } \\ \delta_{\text {vert }}^{e} & 0.0007 & 0.0001 \\ \delta_{\gamma}^{e} & -0.0009 & -0.0003 \\ \delta_{v e r t}^{f} & 0.0014 & 0.0001 \\ \delta_{\gamma}^{f} & -0.0012 & -0.0001 \\ \delta_{\text {vac }} & 0.0011 & -0.0002\end{array}$

Total $\quad 0.0016 \quad 0.0004$

\section{A.3 Bhabha Scattering}

The cuts made in the Bhabha event selection will effect the radiative correc- 
tion by imposing limits on the $q^{2}$ and radiated photon energy.

The exact expression for vacuum polarization in Bahbha scattering is rather complicated and is given by

$$
\begin{aligned}
\delta_{v a c}= & \frac{1}{\frac{d \sigma}{d n}} \frac{-\alpha^{2}}{8 \chi^{2}}\left[\left(2-2 \chi+\chi^{2}\right) \Re \Pi(-8 \chi)-\chi\left(1-\chi^{2}\right)(\Re \Pi(-s \chi)+\Re \Pi(s))\right. \\
& \left.+\chi^{2}\left(1-2 \chi+2 \chi^{2}\right) \Re \Pi(s)\right]
\end{aligned}
$$

where $\chi=\sin ^{2} \frac{\theta}{2}$. For the purposes of this Appendix we will use

$$
\delta_{v a c} \approx 2 \Re I(-8 \chi)
$$

and say that the numbers given should be used for comparison purposes and not necessarily for computational purposes.

If the initial state photon of energy $k$ goes down the beam pipe ( a good assumption since $\frac{d \sigma}{d \Omega_{k}} \sim \frac{1}{\sin ^{2} \theta_{k}}$ ), then the following relation applies:

$$
k=\frac{2 E_{\text {beam }}}{1+\frac{\sin \theta+\sin (\theta+\xi)}{\sin \xi}}
$$

where $\xi$ is the acollinearity angle between the electron and positron and $\theta$ is the smaller of the two particle polar angles. In this experiment $\xi \leq 10^{\circ}$ implies $k_{\text {max }}^{e} \approx 0.18 E_{\text {beam }}$.

In final state radiation the photon has the same tendency as initial state radiation to follow the parent particle's original direction. Unlike the initial state case, a very hard photon can be emitted and the event will still satisfy the acollinearity cut and other cuts. Thus final state radiation has $k_{\text {max }}^{f} \approx E_{\text {beam }}$. The value of $C_{\alpha^{3}}$ for Bhabhas with $\theta>55^{\circ}$ is 1.025 which is identical to $C_{\text {leading }}$ to 3 decimal places. 
The acollinearity angle of Bhabha events is related to the momentum imbalance of the electron and positron along the beam axis. In single photon emission the momentum imbalance is the bremsstrahlung photon momentum. The Monte Carlo programs we have used to model bremsstrablung do not include multiple photon emission and this will lead to an error in the cross section calibration. In the case of multiple photon emission in which both the electron and positron can radiate the momentum imbalance is given by:

$$
Y=y_{e^{+}}-y_{e^{-}}
$$

where $y_{e^{ \pm}}$is the total photon energy normalized by the beam energy from the $e^{ \pm}$. To study the effect of multiple photon emission the acollinearity distribution is integrated over all allowed acollinearities. The expression for the acollinearity distribution is given by the convolution of the energy distributions for single photon emission by an electron and can be written as,

$$
\begin{aligned}
A(Y) & =\int_{-Y}^{Y} d y \int_{0}^{1} d y_{e^{-}} \int_{0}^{1} d y_{e^{+}} f\left(y_{e^{-}}\right) f\left(y_{e^{+}}\right) \delta\left(y_{e^{+}}-y_{e^{-}}-Y\right), \\
f(y) & =t \frac{1}{y}\left(1-y+\frac{y^{2}}{2}\right) F(y), \\
F(y) & =\int_{0}^{y} f\left(y^{\prime}\right) d y^{\prime}, \\
& =y^{t} e^{-t\left(x-x^{2} / 4\right)} \\
t & =\frac{1}{2} \frac{2 \alpha}{\pi}\left(\ln \frac{q^{2}}{m_{e}^{2}}-1\right),
\end{aligned}
$$

where $F(y)$ is the probability of a emitting a photon of normalized energy $y$ and $t$ is $\frac{1}{2}$ of the usual "radiator" to take into account radiation from either particle. $A(Y)$ can be written more simply as

$$
\begin{aligned}
A(Y) & =2\left[\int_{0}^{1} f(y) F(y) d y-\int_{Y}^{1} f(y) F(y-Y) d y\right] \\
& =F^{2}(1)-2 \int_{Y}^{1} f(y) F(y-Y) d y .
\end{aligned}
$$


The integral must be computed numerically. The results are based on large angle Bhabha-scattering $\left(q^{2}=-307 \mathrm{GeV} / \mathrm{c}^{2}\right)$ using the exponential model (the leading $\log$ should give quite similar results). The ratio of $A(y)$ to $F(y)$ gives the correction due to the assumption that the missing momentum is equal to the photon energy. This assumption introduces an error into the radiative correction of $0.35 \%$. In order to compensate for this error the cross section (luminosity) measurement should be increased (decreased) by $0.35 \%$.

\section{A.4 Multi-Hadron Production}

In the case of multi-hadron production there is no acollinearity cut and $k_{\max }^{e}$ can be quite close to the beam energy. As shown in Appendix B events with very hard photons are accepted with a low efficiency. Final state radiation can be up to the kinematic limit. For light quarks $k_{\max }^{f} \approx E_{\text {beam }}$.

Due to the contribution of initial state radiation a significant number of events will be accepted which were produced at a center-of-mass energy well below the nominal value of the center-of-mass energy. Thus it is important to know the value of $R$ at lower energies also. The uncertainty of the lower energy measurements will propagate through the analysis and affect the precision of the nominal energy $R$ value. The acceptance of highly radiative events drops off sharply for $k^{e}>\frac{1}{2} E_{b e a m}$ so we set $k_{m a x}^{e}=0.7 E_{b e a m}$ for calculational purposes. The variation of $R$ due to uncertainties in the low energy measurement of $R$ is given by:

$$
\delta R(s) \approx \frac{2 \alpha}{\pi}\left(\ln \frac{s}{m_{e}^{2}}-1\right) \int_{k_{\min }^{e}}^{k_{\operatorname{maz}}^{e}} \frac{1}{1-k}\left(1-k+\frac{k^{2}}{2}\right) \delta R(s(1-k)) \frac{d k}{k}
$$




$$
\delta R(8) \approx \frac{2 \alpha}{\pi}\left(\ln \frac{8}{m_{e}^{2}}-1\right)\left[\ln \frac{k_{\max }^{e}}{k_{\min }^{\mathrm{e}}}+\frac{1}{2} \ln \left(\frac{1-k_{\min }^{e}}{1-k_{\max }^{e}}\right)-\frac{k_{\max }^{e}-k_{\min }^{e}}{2}\right] \delta R(\langle s\rangle)
$$

where $k_{\min }^{e}$ is set by the center-of-mass energy above which we think $\mathrm{R}$ is a constant to the precision of this experiment. Table A-5 shows $\delta R(s) / \delta R((s))$ for various values of $k_{\min }^{e}$ and $k_{\max }^{e}$. To obtain the uncertainty due to the error in the low energy measurement we should use the best value ${ }^{3}$ of $\delta R(\langle s\rangle), \delta R(\langle s\rangle)=$ \pm 0.10 .

\section{Table A-5}

Uncertainty in $R$ due to errors in low energy $R$ measurements

$\begin{array}{ccccc}k_{\min }^{e} & k_{\max }^{e} & \left\langle E_{c m}\right\rangle & \delta R(s) / \delta R(\langle s\rangle) & \delta R(s) \\ 0.1 & 0.5 & 25.0 & 0.165 & 0.017 \\ 0.2 & 0.5 & 23.7 & 0.097 & 0.010 \\ 0.2 & 0.6 & 22.8 & 0.121 & 0.012 \\ 0.2 & 0.7 & 22.1 & 0.145 & 0.015 \\ 0.3 & 0.7 & 20.7 & 0.104 & 0.011 \\ 0.4 & 0.7 & 19.5 & 0.073 & 0.008\end{array}$

In this experiment $\delta R(8)=0.02$ implies an error in $R$ due to the uncertainty in lower energy $\mathrm{R}$ measurements of $\approx \frac{1}{2} \%$.

Final state radiation is much easier to deal with than initial state radiation. This can be seen with the Lee-Nauenberg theorem ${ }^{4}$ which states that if one sums over all degenerate initial states then all mass singularities cancel out to all orders in perturbation theory, except those from vacuum polarization. In 
an $e^{+} e^{-}$experiment the machine selects the energy of the particles so there is no summing over all degenerate states and the mass singularity in initial state radiation remains. In the final state all degenerate states are summed over and after cancellation the result is $\approx \frac{1}{2} \%$ correction due to final state radiation.

The radiative correction given by the Monte Carlo must be adjusted to take into account the leading $\log$ terms. The effects of the cuts on the radiative corrections is calculated by convoluting the differential cross section with the efficiency of selection with respect to the bremsstrahlung energy

$$
\sigma^{r . c .}=\int_{0}^{E_{b e s m}} \frac{d \sigma^{r . c .}}{d k} \epsilon(k) d k
$$

where r.c. $=\alpha^{3}$ or ${ }_{\log }^{\text {leading }}$,

$$
\frac{d \sigma^{\alpha^{8}}}{d k}=\sigma^{\alpha^{2}} \frac{d C_{\alpha^{3}}}{d k}
$$

and similarly

$$
\frac{d \sigma^{\text {leading }}}{d k}=\sigma^{\alpha^{2}} \frac{\begin{array}{c}
d C_{\text {leading }} \\
\log ^{\prime}
\end{array}}{d k}
$$

$\epsilon(k)$ is the efficiency calculated with a Monte Carlo. The radiative corrections given by the Monte Carlo are to $O\left(\alpha^{3}\right)$ only. The leading log terms are taken into account by multiplying the Monte Carlo radiative correction by

$$
\frac{\sigma^{\text {leading }}}{\sigma^{\alpha^{3}}}=1.017
$$

\section{A.5 Conclusion}

Table A-6 gives summarizes the errors due to radiative effects. The total error is $\sim \frac{1}{2} \%$ for Bhabha scattering and $\sim 1 \%$ for multi-hadrons. 
Table A-6

Final systematic uncertainties due to radiative effects for large angle Bhabha scattering $\left(\left\langle q^{2}\right\rangle=-307(\mathrm{GeV}) / \mathrm{c}^{2}\right)$ and for multi-hadrons $\left(\left\langle q^{2}\right\rangle=780(\mathrm{GeV}) / \mathrm{c}^{2}\right)$.

Effect

Low energy $R$

Vacuum

polarization

Non-leading logs

Kinematic enhancement

"New physics"

Total
Bhabha(\%) multi-hadron (\%)

0.5

0.3

0.4

0.04

0.16

$0.1 \quad 0.4$

$\approx 0$

0.1

0.3

The final correction used to adjust the Bhabha cross section is $1.000 \pm 0.003$ and for the multi-hadron cross section the correction is $1.017 \pm 0.008$.

In conclusion a detailed examination of radiative corrections applied to $\mathrm{R}$ measurements has been made. Including leading log terms provides some higher order terms and shows that the lowest order radiative correction is quite adequate for most purposes. The major uncertainty in $\mathrm{R}$ due to radiative corrections results from the error in lower energy measurements of $R$. 


\section{REFERENCES}

1. Lynch, H. L., MAC Note 640a (unpublished), 1983.

2. Tsai, Y. S., SLAC Report No. SLAC-PUB-3129, (1983).

3. Bartel, W., et al., DESY Report No. DESY 83-050, (1983).

4. Lee, T. D., and M. Nauenberg, Phys. Rev., B133,1549 (1964). 


\section{Appendix B}

\section{Monte Carlo Methods}

Physical processes occur in a statistical manner so it is natural to model them in a statistical fashion. The modelling method usually employed is the Monte Carlo method. The Monte Carlo method uses a model of the process under study and creates all possible configurations in a statistical manner, just as occurs in reality. This experiment needs two types of Monte Carlos, one for the physics processes and one for the response of the detector.

\section{B.1 Physics Monte Carlos}

\section{Primordial Quark Generator}

The primordial quark generator program runs by first generating a quark anti-quark photon state from the decay of a virtual photon or $Z^{0}$. The $q q \gamma$ states were generated with the Kleiss $\mu \mu \gamma$ generator ${ }^{1}$ with appropriate substitutions for quarks. The program includes QED effects to $O\left(\alpha^{3}\right)$. The contribution to the cross section from initial state bremsstrablung depends on the hard photon cut-off. Figure B-1 shows the bremsstrahlung energy spectrum and the event selection efficiency as a function of bremsstrahlung photon energy. In practice such hard photon events are found with low efficiency so that the acceptance is only weakly dependent on the photon energy cut-off. The final state radiation 

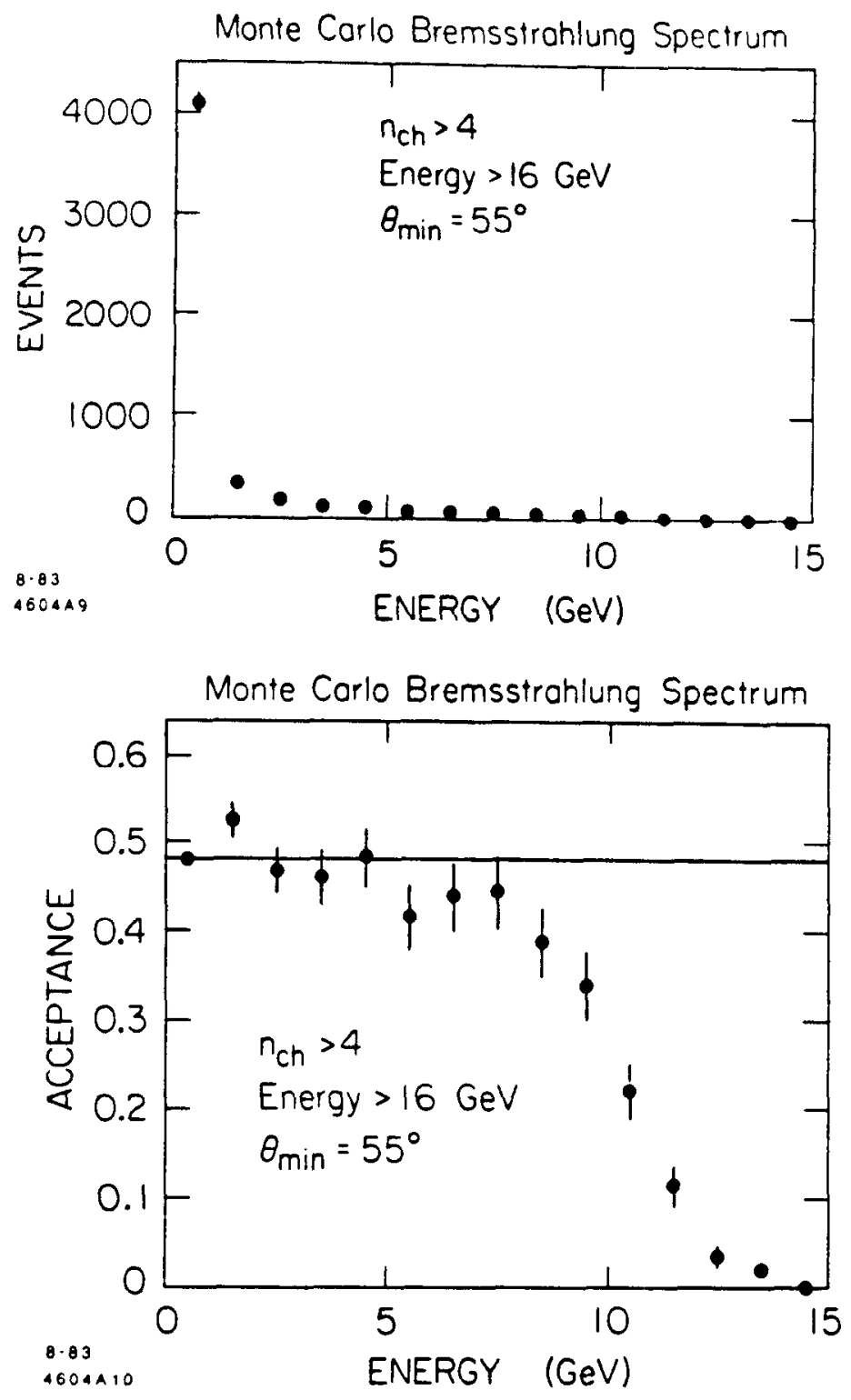

Figure B-1: Bremsstrahlung energy spectrum (top). Acceptance efficiency for events with $n^{\text {ch }}>4,55^{\circ}<\theta_{p_{t h \text { ret }}}<80^{\circ}$ as a function of bremsstrahlung energy (bottom). The solid line is the acceptance of the fiducial volume. 
process is almostly completely cancelled by the final state vertex correction as explained in Appendix A. Final state radiation is complicated by the fact that quarks interact strongly which has unknown effects on the final state QED radiative corrections. One might argue that since the strong interaction is stronger than the electromagnetic interaction, the strong processes will occur on a faster time scale and reduce the apparent QED corrections in the final state but since the final state contribution is much smaller than the precision of the experiment the effect is ignored. Higher order QED processes can be treated in an approximate way summing leading logs as detailed in Appendix A. The bremsstrahlung photon energy cut-off was set at the kinematic limit for each quark.

In addition to pure QED there is the Weak interaction and its interference with QED. The Standard Model has the Weak force transmitted via an intermediate vector boson, the $Z^{0}$ in our case. Using the Siandard Model for the fermion-boson coupling and typical values for $M_{Z^{0}}$ and $\sin ^{2} \theta_{w}$ the effect due to Electro-Weak interference is only about $4 / 10 \%$ at $29 \mathrm{GeV}$ center-of-mass energy and is included in the Monte Carlo event generators.

After a quark flavor is chosen the program decides if the event is going to have 2-,3-, or 4-jets. The quarks and gluons are then fragmented into hadrons and the unstable particles are allowed to decay. An array is filled with particle 4-vectors and the event is ready to be sent through the detector Monte Carlo and finally the analysis programs.

Two photon Event Generator 
A hadronic final state need not be the result of an $e^{+} e^{-}$annihilation. If both the $e^{+}$and $e^{-}$radiate a photon then these photons can interact to produce a hadronic final state. The two photon process can be modelled in two ways corresponding to different regimes of momentum transfer, $q^{2}$.

For lower $q^{2}$ the hadronic component of the photon (recall that a real photon is a bare Dirac photon plus a superposition of particle-antiparticle pairs including quarks) behaves like a vector meson. The vector mesons then interact to form a hadronic final state. This model is called the Vector Dominance Model (VDM). Events in the VDM have a very sharp drop-off in $p_{\perp}$ relative to the beam axis.

In the other model, called the hard-scattering model the photons couple directly to quarks. The cross section for this process has the behavior:

$$
\sigma \sim 3 \sum_{\substack{\text { parark } \\ \text { flavors }}} e_{q}^{4}
$$

where the $e_{q}^{4}$ comes from the fact that there are 2 quark vertices in the $2 \gamma$ process.

As mentioned before, the $2 \gamma$ process is the largest background to the annihilation cross section. The Vermasseren generator ${ }^{2-3}$ was used to generate the 6 lowest order diagrams for $e^{+} e^{-} \rightarrow e^{+} e^{-}+$hadrons, using $u, d, 8$, and $c$ quarks. The Ali program was then used to fragment the quarks. Because most of the $2 \gamma$ cross section occurs outside the cuts used in the analysis, some cuts were applied to the generator so that a reasonable sample of events could be obtained in a finite amount of computer time. The cuts for $u, d$, s quarks were: energy of the quark pair $>4 \mathrm{GeV}$ and both quarks with $|\cos \theta|<0.98$ relative to the beam axis. For $c$ quarks no kinematic cuts were applied. 


\section{Hadronization of Quarks}

Two annihilation multi-hadron Monte Carlo programs were used to model the quark fragmentation. One of the Monte Carlo programs used was the program of Ali et al. ${ }^{4}$ The other Monte Carlo used was the Lund University program. ${ }^{5}$ The Ali program includes the final states $q \bar{q}, q \bar{q} g, q q q \bar{q}$, and $q \bar{q} g g$. The quark hadronization proceeds by the incoherent jet model. In the Lund program the QCD affects are to $O\left(\alpha_{s}\right)$ only and use the string model for hadronization. The acceptance of events using the two fragmentation schemes was within the statistical limits of the number of events generated.

In the independent (or incoherent) jet model of quark fragmentation ${ }^{6}$ an original pair of quarks $q_{0} \bar{q}_{0}$ create a color field in which new quark pairs $q_{i} \bar{q}_{i}$ are produced. These quark pairs combine to form primary mesons, $m_{i}$, with quark content $q_{i} \bar{q}_{i+1}$. The production of primary mesons continues until all the momentum along the original quark direction is used up. The primary mesons are then allowed to decay according to their properties. The quark pairs produced in the color field are $u u, d \bar{d}$, and $s \bar{s}$ in the ratio 2:2:1. Baryon production is not included in the original Feynman-Field recipe but can be incorporated. Figure B-2 shows the hadronization of quarks using the Feynman-Field model including baryon production.

In the incoherent jet model with the assumption of very high momentum, all distributions scale and depend only on $p_{h a d} / p_{q}$. This implies a complete knowledge of the structure of a quark jet determined by an arbitrary function

and three parameters describing flavor, primary meson spin, and $p_{\perp}$ relative 


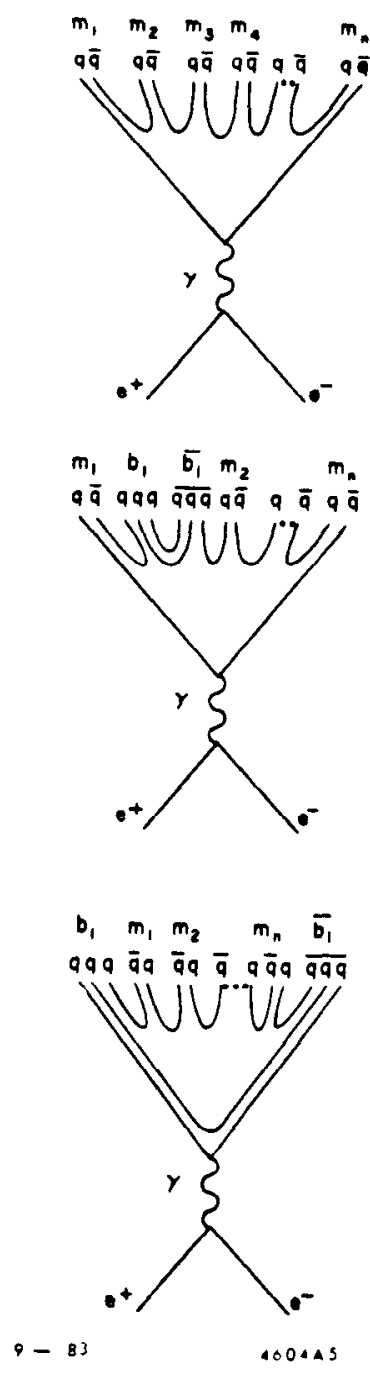

Figure B-2: Quark fragmentation in the incoherent jet model. 
to the jet. The arbitrary function is the probability a primary meson leaves a fraction of momentum to the remaining cascade and has been chosen to agree with experimental data. Each quark pair from the sea has a $p_{\perp}$ distributed according to $e^{-\left(p_{\perp} / 2 \sigma_{p_{\perp}}\right)^{2}}$, with $\sigma_{p_{\perp}} \approx 0.3 \mathrm{GeV}$.

Another method for fragmenting quarks comes from Lund University. This method employs the so-called string model in which the two jets are correlated. In the Lund model the $q q$ system behaves as if the quarks were connected by a string whose tension is proportional to the color force between the quarks. The final state hadrons are formed by the creation of $q \bar{q}$ pairs along the string. Gluons are treated as kinks in the string, that is, as a transverse momentum of the string.

\section{Varying Monte Carlo Parameters}

Various parameters exist in the Monte Carlos for tuning the Monte Carlo distributions to the experimentally observed distributions. The parameters in the Ali Monte Carlc are; thrust cut-off, $\alpha_{s}$, the vector to pseudoscalar meson ratio, the $p_{\perp}$ distribution, and the quark fragmentation function. In order to check the effect of variation of the parameters on the acceptance and to save considerable computer time the generated events were put through a fast Monte Carlo program which was optimized to reproduce the multiplicity, energy, and angular distributions of the data. The fast Monte Carlo does not do the detailed energy loss calculations of the full-blown detector Monte Carlo.

The thrust cut-off provides a lower limit to the energy that a real gluon may have. The lower the thrust cut-off the greater the fraction of $q q$ events. To chcck 
the effect of thrust cut-off on acceptance a set of events with $T_{\max }=0.87$ was generated. To get a correlated set of events with a lower $T_{\max }$ the events with $\mathrm{T}_{\max }<\mathrm{T}<0.97$ were assigned a weight of zero and the $q \bar{q}$ events $(\mathrm{T}=1)$ were assigned a weight such that the total weight of the entire sample is the same no matter what the value of $T_{\max }$. With $\mathrm{T}_{\max }$ set to $0.83,0.05,0.87$ the variation in acceptance was $\frac{1}{10} \%$.

To check the variation of acceptance with $\alpha_{s}$ the events were reweighed according to whether they were $q \bar{q}, q \bar{q} g$, or $q \bar{q} g g$ to effectively change $\alpha_{s}$. With $\alpha_{s}=0.120,0.165,0.220$ the variation in acceptance was $\frac{1}{2} \%$.

The probability that a quark fragments and forms a primordial pseudoscalar meson rather than a vector meson effects the acceptance because vector mesons are more likely to decay into charged particles. To estimate the effect on the acceptance of the pseudoscalar to vector ratio, two Monte Carlo event files were generated; in one file the quarks were allowed to fragment only into vector mesons (eg. $\rho, \omega, \phi)$ and in the other file the quarks were allowed to fragment only into pseudoscalar mesons (eg. $\pi, \eta, D$ ). We assume that the acceptance varies linearly with the fraction of pseudoscalar mesons produced, $\mathrm{P} /(\mathrm{P}+\mathrm{V})$. The real world value of $\mathrm{P} /(\mathrm{P}+\mathrm{V})$ is a linear combination of the two extreme cases generated, $P /(P+V)=0,1$. The acceptance varies by $2.7 \%$ between the extreme values of $\mathrm{P} /(\mathrm{P}+\mathrm{V})$. For reasonable variation ${ }^{7}$ of $\mathrm{P} /(\mathrm{P}+\mathrm{V})$, about \pm 0.15 , the acceptance changes by $\approx \frac{4}{10} \%$.

The effect of $\sigma_{p_{\perp}}$ is in the broadness of jets (cf. Ch 4), the larger the value of $\sigma_{p_{\perp}}$ the broader the jet. The broadness of a jet will effect the acceptance by 
causing more or less charged particles to be within the drift chamber acceptance which affects the multiplicity distribution. To measure the variation of acceptance with $\sigma_{p_{\perp}}$ three Monte Carlo runs were made with different values of $\sigma_{p_{\perp}}$. The values run were $\sigma_{p_{\perp}}=0.15 \mathrm{GeV}, 0.30 \mathrm{GeV}$, and $0.45 \mathrm{GeV}$. The variation in acceptance from the standard value of $0.30 \mathrm{GeV}$ was -.003 and +.005 for $.15 \mathrm{GeV}$ and $.45 \mathrm{GeV}$ respectively. It should be noted that the uncertainty ${ }^{8}$ in $\sigma_{p_{\perp}}$ is only about 0.04 so that for a reasonable variation of $\sigma_{p_{\perp}}$ the change in acceptance is about .002 .

The effect of heavy quark fragmentation function on acceptance was also studied. Events were generated with a fragmentation function $1-x$ for $c$ quarks and constant fragmentation function for $b$ quarks. A set of events were also generated according to

$$
D_{q}(z) \sim\left[z\left(1-\frac{1}{z}-\frac{\epsilon_{q}}{1-z}\right)\right]^{-1}
$$

The latter function provides a reasonable fit to experimental data ${ }^{9}$. The difference in acceptance between the two models was about $0.8 \%$.

\section{$\tau$-pair Event Generator}

Another background comes from t-pair production. The $>4$ charged tracks cut removes about $98 \%$ of the $\tau$-pairs (with $\mathrm{BR}[\tau \rightarrow \geq 3$ charged prongs $=1 / 7]$ ). The Kleiss $\tau$-pair generator was used to measure the effects of the cuts and the promotion of 4-prong events to 6-prong events resulting from $\gamma$ conversion. Events with $n^{\text {ch }}>4$ and $\mathrm{E}<16 \mathrm{GeV}$ in the angular acceptance have a cross section of $0.20 \mathrm{pb}$ and with $\mathrm{E}>16 \mathrm{GeV}$ the cross section is $1.02 \mathrm{pb}$.

\section{Bhahba Event Generator}


The luminosity in this experiment is measured with Bhabha events. A computer program using the results of Berends ${ }^{10}$ has been used to model electronpositron scattering. QED effects to $O\left(\alpha^{3}\right)$ are included as are lowest order weak effects. In order to save the large amount of computer time that would be used simulating the showering process the Bhabha events were not put through the detector Monte Carlo. The central drift was modelled using the known resolutions of the chamber.

\section{B.2 Detector Monte Carlo}

Once the particle four-vectors are produced they must be propagated through the apparatus. The modelling of the apparatus includes the proper dimensions and compositions of various detector components. The output of the detector Monte Carlo produces an event record exactly like that of a data record; pulse heights, drift times, etc. The Monte Carlo is tuned to fit the experimentally observed distributions, eg. mean energy, and the number of random hits in the central drift chamber.

\section{Central Drift Monte Carlo}

Two models of the central drift chamber have been used to simulate the performance of the actual chamber. The first model was optimized with respect to $\mu$-pairs. The drift times and extra hit distributions were adjusted to be in agreement with the experimentally observed $\mu$-pair distributions. If the drift chamber behaves in a linear fashion then all the distributions should scale accordingly for multi-hadron events. This is not the case (with LUND82 String Model Monte Carlo as physics input), the central drift Monte Carlo is a cleaner 
device and is better at reconstructing tracks than the actual chamber. The second model (with Ali Feynman-Field Monte Carlo) was optimized with respect to multi-hadron events. This model uses the gross distributions such as hits per layer and average hits per track for comparison with the data rather than the more fundamental distributions as in the first model. The second model reproduces more nearly the performance of the actual chamber with respect to multi-hadrons. The manifestation of drift chamber performance is in the angular and charged multiplicity distributions. As explained in the analysis chapter the Monte Carlo angular distribution is in very good agreement with the data and the Monte Carlo charged multiplicity distribution is adjusted to bring it into agreement with the data.

Simulation of Electromagnetic Showers

More than half the energy deposited in MAC is in the electromagnetic shower chamber and a large fraction of this energy is the result of electromagnetic showers. The method for simulating electromagnetic showers ${ }^{11}$ is a Monte Carlo simulation using computer code called Electron Gamma Shower. ${ }^{12}$ The EGS program models the transport of electrons, positrons, and photons by simulating the physical processes as closely as possible. Theoretical and empirical formulas are used to compute the necessary physical quantities. The major contributors to electromagnetic showers are bremsstrahlung and pair production. In fact the contributions of these two processes are comparable. Other processes modify the distributions of a shower and include; multiple Coulomb scattering, ionization energy loss through inelastic collisions with atomic electrons in a medium, Compton effect, and Photoelectric effect. Other effects included in the program are; the 
Chudakov effect which results in a lower energy loss from an electron-positron pair because of the interference of the electromagnetic fields of the two particles, the Landau-Pomeranchuk effect which reduces the effect of bremsstrahlung when an electron is scattered by the electric field of a nearby by atom, $\mathscr{C}$ erenkov radiation which is emitted when a charged particle passes through a medium faster than the speed of light in that medium, and transition radiation which is emitted when a charged particle crosses a boundary between two different media.

The EGS program transports a particle until an interaction occurs, or until its energy drops below a cutoff energy, or until it leaves the region of interest. If an interaction occurs then any other particles produced are added to the list of particles to be transported. The transport process continues until all the particles are either below the cut-off energy or outside the region of interest. After the shower is completed there is a user-definable program which contains the information to be used in the event. In this experiment the only quantities of interest are the magnitude and location of the shower.

\section{Simulation of Hadronic Showers}

A significant fraction of the energy lost in MAC is in the hadron calorimeter resulting from hadronic showers. The program used to model hadronic showers ${ }^{13}$ is called High Energy Transport $\underline{\text { Code. }}{ }^{14}$ The program will transport neutrons, protons, charged pions, and muons. The energy loss mechanisms used are; atomic ionization and excitation, multiple Coulomb scattering, range straggling, $\pi$ - and $\mu$-decay, and nuclear interactions. HETC uses analytic formulas and empirical results for the necessary physical quantities. 
HETC uses an intranuclear collision model for hadron-nucleon interactions. A high energy hadron will have a small enough deBroglie wavelength so that the interaction of a high energy hadron with a nucleus will involve only the hadron and single nucleon rather than the entire nucleus. Since this is an interaction inside nuclear matter the collision products must obey the Pauli Exclusion Principle. The interaction may produce secondary particles which can undergo further interaction in the nucleus. After the intranuclear cascade is completed, the nucleus is left in an excited state with the excess kinetic energy assumed to be equally distributed among its constituents. The excess energy is shed by a statistical evaporation of particles; neutrons, protons, deutrons, tritons, helium nuclei, and heavier nuclei. For heavy nuclei fission can occur during the evaporation phase. When particle emission is no longer energetically possible, the residual nucleus de-excites by photon emission and the shower stops. As in the electromagnetic case the only quantities of interest are the magnitude and location of the shower.

\section{B.3 USING The Monte CARLOS}

More than $\mathbf{4 0 0 0 0}$ Monte Carlo events from all processes modelled were used in this analysis for computing backgrounds, efficiencies, etc. Due to finite computer storage and time the Monte Carlo events correspond to less integrated luminosity than is actually used in the analysis. The Monte Carlo method can be a valuable tool if its limitations are understood and respected. Chapter 4 on Data Analysis has shown that this analysis is only weakly dependent on precise Monte Carlo modelling. 


\section{REFERENCES}

1. Berends, F. A., R. Kleiss, and S. Jadach, Nucl. Phys., B202, 63, (1982).

2. Bhattacharya, R., Smith, J., and Grammer, G., Phys. Rev. D15, 3267 (1977).

3. Smith, J., Vermasseren, J. A. M., and Grammer, G., Phys. Rev. D15, 3280 (1877).

4. Ali, A., E. Pietarinen, and J. Willrodt, DESY Report No. DESY T$80 / 01,(1980)$.

5. Sjostrand, T., "A Monte Carlo Program for Quark and Gluon Jet Generation”, Lund University Report No. LU-TP 80-3, (1980).

6. Field, R. D. and R. P. Feynman, Nucl. Phys., B136, 1, (1978).

7. Brandelik, R., et al., Phys. Lett., 94B, 437, (1980).

8. Brandelik, R., et al., Phys. Lett., 94B, 437, (1980).

8. Fernandez, E., et al., Phys. Rev. Lett., 50, 2054 (1983).

10. Berends, F. A., R. Gastmans, and K. J. F. Gaemers, Nucl. Phys. B68, 541, (1974).

11. Armstrong, T. W., Computer Techniques in Radiation Transport and Dosimetry, edited by W. R. Nelson and T. M. Jenkins. (Plenum Press, N. Y., (1980).

12. Ford, R. L., and W. R. Nelson, SLAC Report No. SLAC-210, (1978).

13. Armstrong, T. W., Computer Techniques in Radiation Transport and Dosimetry, edited by W. R. Nelson and T. M. Jenkins. (Plenum Press, N. Y., (1980).

14. Armstrong, T. W., and Chandler, K. C., Nucl. Sci. and Engr., $\underline{49}, 110$ (1972). 


\section{Appendix C}

\section{Luminosity Measurement}

The luminosity measurement has been made using a well-understood process in a well-understood region of the detector. Specifically, Bhabha scattering $\left(e^{+} e^{-} \rightarrow e^{+} e^{-}\right)$in the central region of the detector has been used.

The events are selected off-line according to the following criteria:

1) exactly 2 inner drift tracks;

2) $>5.7 \mathrm{GeV}$ in the shower chamber;

3) $<10^{\circ}$ acollinearity angle;

4) $>$ each track $55^{\circ}$ from the beam axis as measured by the inner drift;

5) the azimuthal angle of $\overrightarrow{p_{1}}-\overrightarrow{p_{2}}$ must be at least $4^{\circ}$ from the edge between shower sextants. The final cut is to eliminate events near the edges between sextants where there is dead area.

Pictures of a sub-sample of the selected events are scanned to determine the level of background contamination which is found to be $1.2 \pm 0.8 \%$. The efficiency of the inner drift chamber is checked by selecting and scanning events with collinear showers. The efficiency for finding exactly 2 tracks in the inner drift chamber is $\mathbf{9 4 . 0} \pm \mathbf{0 . 7 \%}$. Most of the inefficiency comes from radiated 
photons which have pair converted in the beam pipe to produce extra tracks. A shower energy spectrum shows that the energy cut is well below the energy that can be expected from an actual Bhabha event.

The efficiency of events passing these cuts and the scanning was determined by looking at the hardware triggers of these events. One trigger used is the total energy trigger which finds $\mathbf{8 8 . 8 \%}$ of the selected Bhabha events. Also used is the back-to-back scintillation trigger which is only $44.0 \%$ efficient because the scintillators are behind the shower chamber and are not meant to be used as a primary Bhabha trigger. Other hardware triggers also trigger on Bhabha events and are $97.0 \%$ efficient. Thus the probability of failing to trigger on a central Bhabha event at the hardware level is $(1-.988)(1-.440)(1-.970)=.0002$ and no correction is made.

In order to calibrate the Bhabha cross section to obtain a luminosity the Kleiss Monte Carlo is used. It includes effects to $O\left(\alpha^{3}\right)$ and electro-weak effects. Because of the large number of events $(\approx 100,000)$ needed to obtain reasonable precision the events are not put through the detector Monte Carlo. As mentioned above the energy cut is well below what can be expected from real Bhabha events, so there is no loss of precision from not modelling the showering process. The important cut is the angular cut and the angular resolution of the drift chamber has been modelled by randomly smearing the generated angles by the known drift chamber resolution. At $\sqrt{8}=29 \mathrm{GeV}$ the cross section is $0.60 \mathrm{nb}$. Using the higher order corrections as described in Appendix A increases the Monte Carlo cross section by $0.35 \%$. The major contribution to the higher order correction is from the change in the acollinearity distribution due to the fact that photons 
may be emitted by both the electron and positron in the same event which is not modeled by the Monte Carlo.

The present analysis uses a sample of large-angle Bhabha scattering events which contains $40426 \pm 0.5 \%$ (stat) events. All the data were taken with $14.5 \mathrm{GeV}$ colliding beams expect for $2.1 \mathrm{pb}^{-1}$ taken with $14 \mathrm{GeV}$ beams. The calculated luminosity is $80.81 \mathrm{pb}^{-1} \pm 1.60 \%$ (syst.).

Other measurements of the luminosity, all less precise than the measurement being used, have been made. One such measurement was made using Bhabha events at smaller angles including the endcap regions. The small- and large-angle Bhabha analyses are consistent within the $5 \%$ systematic error of the endcap measurement. A small angle ( $\sim 30 \mathrm{mrad})$ luminosity monitor, used mainly for on-line purposes, has also been used to measure the integrated luminosity. Within the fairly large errors $(\sim 5 \%)$ of the device the luminosity measurement agrees with the large angle Bhabha measurement. Also, the luminosity was measured with collinear $\mu$-pair events. In addition to an acollinearity cut $\left(<10^{\circ}\right)$, each event was required to have $>8 \mathrm{GeV} / \mathrm{c}$ scalar momentum sum for the two verticizing innner drift chamber tracks. Further, the inner drift tracks must point to minimum ionizing tracks in the hadron calorimeter or to an outer drift chamber track. Additional cuts on trigger counter timing and vertex position remove cosmic rays. The error in this method of luminosity measurement is about $3 \%$. The weighted average of the additional luminosity measurements is in good agreement with the large angle Bhabha measurement and has a comparably sized systematic error. 
In conclusion the single best measurement of integrated luminosity is used in the analysis. To improve the luminosity measurement requires better modelling of the central drift chamber and a better understanding of the background contamination. 


\section{Bibliography}

Ali, A, E. Pietarinen, and J. Willrodt, DESY Report No. DESY T-80/01, 1880.

Armstrong, T. W., Computer Techniques in Radiation Transport and Dosimetry, edited by W. R. Nelson and T. M. Jenkins. (Plenum Press, N. Y., 1880).

Armstrong, T. W., and Chandler, K. C., Nucl. Sci. and Engr., 모, 110 (1972).

Barber, D. P., et al., Phys. Rev. Lett., 42, 1113 (1970).

Barnett, R. M., M. Dine, and L. McLerran, Phys. Rev. D, 22, 584 (1980).

Bartel, W., et al., DESY Report No. DESY 81/048, 1981.

Bartel, W., et al., DESY Report No. DESY 83-050, 1983.

Berends, F. A., R. Gastmans, and K. J. F. Gaemers, Nucl. Phys. B68, 541 (1974).

Berends, F. A., R. Kleiss, and S. Jadach, Nucl. Phys., B202, 63 (1982).

Berger, C., et al., Phys. Lett., 99B, 292 (1981).

Bhattacharya, R., Smith, J., and Grammer, G., Phys. Rev. D15, 3267 (1977). 
Bjorken, J. D. and Pachos, G. A., Phys. Rev., 185, 185 (1969).

Brandelik, R., et al., Phys. Lett., 88B, 171 (1879).

Brandelik, R., et al., Phys. Lett., 94B, 437 (1980).

Brandelik, R., et al., Phys. Lett. 107B, 290 (1881).

Brandelik, R., et al., Phys. Lett., 113B, 499 (1880).

Brodsky, S. J., T. Kinoshita, and H. Terazawa, Phys. Rev. D, 4, 1532 (1971).

Charpak, G. and F. Sauli, Nucl. Instr. Meth., 162, 405 (1979).

Fernandez, E., et al., Phys. Rev. Lett., 50, 2054 (1983).

Field, R. D. and R. P. Feynman, Nucl. Phys., B136, 1 (1878).

Ford, R. L., and W. R. Nelson, SLAC Report No. SLAC-210, 1978.

Ford, W. T., "The MAC Calorimeters and Aplications", in Proceedings of the International Conference on Instrumentation for Colliding Beam Physics, SLAC Report-250, 174, 1882.

Ford, W. T., et al., "Physics with the MAC Detector", SLAC Report No. SLAC-2886, 1882.

Gordon, H, A. and S. D. Smith, "Sampling Calorimeters in High Energy Physics", in Proceedings of the SLAC Summer Institute, 241, 1980.

Heinzelman, G., "Recent Results from JADE at PETRA ", in Proceedings of the 21st In iernational Conference on High Energy Physics, Paris, July 26-31, 1982.

Heltsley, B. K., PhD. thesis (unpublished), 1983. 
Kobayashi, M. and K. Maskawa, Progr. Theor. Phys.,49, 652 (1973).

Lee, T. D., and M. Nauenberg, Phys. Rev., B133, 1549 (1964).

Lynch, H. L., MAC Note 640a (unpublished), 1983.

Mess, K. H. and B. H. Wiik, in Recent Results in Electron-Positron and Lepton-Hadron Interactions, DESY 82-011, 1982.

Ritson, D. M., "Instrumentation", in Proceedings of the SLAC Summer Institute, 177, 1980.

Rossi, B., High Energy Particles, 102-104, (Prentice-Hall, N.J., 1952).

Sjostrand, T., "A Monte Carlo Program for Quark and Gluon Jet Generation”, Lund University Report No. LU-TP 80-3, 1980.

Smith, J., Vermasseren, J. A. M., and Grammer, G., Phys. Rev. D15, 3280 (1977).

Trilling, G., "Mark II Results at PEP", in Proceedings of the 21st International Conference on High Energy Physics, Paris, July 26-31, 1982.

Tsai, Y. S., SLAC Report No. SLAC-PUB-3129, 1983.

Wagner, W., DESY Report No. DESY 80/102, 1880. 